\title{
Multiple Photon Infrared Laser Photophysics and Photochemistry. IV
}

\author{
V. N. BAGRATASHVILI, V. S. LETOKHOV, A. A. MAKAROV \\ and E. A. RYABOV \\ Institute of Spectroscopy, USSR Academy of Sciences, 142092, Troitsk, \\ Moscow Region, USSR
}

(Received July 25, 1983)

This paper is the fourth in series of reviews on multiple photon IR laser photophysics and photochemistry. Unimolecular reactions of MP excited molecules and the isotopic selectivity of MP dissociation are considered.

\section{Contents}

7. Unimolecular Reactions of Molecules Excited by IR Laser Radiation . . . . . . . . . . . . . . . . . 313

7.1. Characteristics of IR multiple photon dissociation . . 314

7.1.1. Experimental methods of IR multiple photon dissociation investigation . . . . . . . 316

7.1.2. Dependence of MP dissociation yield and quantum efficiency on laser pulse energy fluence and absorbed energy . . . . . . . . . . . . . . . 319

7.1.3. Dependence of dissociation yield on IR laser frequency ............... . . . 323

7.1.4. MP dissociation in two-frequency IR field . . 326

7.2. Products of IR multiple photon dissociation and their energy distribution . . . . . . . . . . . . 330 
7.2.1. MP dissociation channels and primary fragments 330

7.2.2. Energy distribution of MP dissociation products 337

7.2.3. Visible luminescence in the case of MP IR excitation of molecules . . . . . . . . . . . . . 342

7.3. Isomerization of multiple photon excited molecules . . 344

7.3.1. Experimental results for various molecules . . . 346

7.3.2. Mechanisms of IR laser-induced isomerization . 352

7.4. Role of collisional processes in IR multiple photon dissociation . . . . . . . . . . . . . 354

7.4.1. Collisions with buffer gas . . . . . . . . 354

7.4.2. Collisions between excited molecules . . . . 359

7.4.3. Secondary reactions . . . . . . . . . . 360

8. Isotopic Selectivity of Multiple Photon IR Dissociation of Molecules . . . . . . . . . . . . . . . . 369

8.1. Characteristics of isotopically-selective MP dissociation 370

8.1.1. Kinetics of enrichment of two-component mixture 370

8.1.2. Experimental methods for selectivity parameters measurements . . . . . . . . . . . . . . 374

8.2. Resonant properties of selectivity . . . . . . . . . . 376

8.2.1. Dependence of MP dissociation selectivity on IR laser frequency . . . . . . . . . . . 376

8.2.2. Selectivity of multiple photon absorption . . 380

8.2.3. Selectivity of MP excitation and dissociation for a small isotope shift . . . . . . . . . . 381

8.3. Ultimate spectral selectivity of MP dissociation. Gas temperature effect . . . . . . . . . . . . . 385

8.4. Role of laser pulse intensity. Two-frequency MP dissociation . . . . . . . . . . . . . . . . . 391

8.5. Effects of collisions on the selectivity of MP dissociation 397 8.5.1. Dependence of selectivity on parent gas pressure and relative isotope content . . . . . . 398

8.5.2. Role of V-V exchange . . . . . . . . . . . . 403

8.5.3. Collisions with buffer gas. Effect of $\mathrm{V}-\mathrm{T}$ and rotational relaxation . . . . . . . . . . 412

8.6. Loss of MP dissociation selectivity in secondary chemical processes 


\section{UNIMOLECULAR REACTIONS OF MOLECULES EXCITED BY IR LASER RADIATION}

The strong excitation of molecules under IR laser radiation has made possible certain novel processes in which irreversible changes of the original substance take place. Excited molecules with large amounts of vibrational energy may undergo different transformations even without collisions. Such transformations are called unimolecular reactions. Among these are the processes of dissociation, isomerization, dissociative ionization, etc. We are going to discuss in this chapter two unimolecular processes, particularly the dissociation and the isomerization of molecules as a result of their MP excitation.

Even in the first works, ${ }^{1,2}$ where the effect of collisionless IR photoexcitation of polyatomic molecules was studied, it was found that molecules could dissociate under the action of IR pulses. The effect of MP dissociation turned out after much work to be nearly universal. Polyatomic molecules with little exception are all able to dissociate when the laser radiation frequency is tuned to the frequency of a vibration active in the IR spectrum. Moreover, it is possible to realize selective action on a single molecular component of a gas mixture. All this gave high promise to the practical value of the effect since it was much simpler to chemically bind the radicals formed in photodissociation than to attempt a high-rate (i.e., before selectivity loss) chemical reaction of the molecule itself even if it is highly vibrationally excited. As a result, one of the most effective methods of laser isotope separation is based on the effect of MP molecular dissociation (see Chapter 8), and laser chemical synthesis is under successful development (see Chapter 9).

The basic processes following MP molecular dissociation are interpreted qualitatively in most cases rather simply. When excited highly enough a molecule reasonantly absorbs laser radiation and, when its energy exceeds the dissociation energy of the weakest bond, it undergoes decomposition. The dissociation rate is highly dependent on the excess molecular energy over the dissociation limit and grows rather fast which restricts the possibility of strong molecular over-excitation relative to the dissociation limit. But the products formed during a laser pulse can in some cases absorb energy and dissociate in their turn. The pectral properties of the process of MP dissociation must evidently be in qualitative agreement with the absorption spectra of 
molecules in a strong IR field considered in the foregoing chapters since a molecule can dissociate only after absorbing a large enough number of quanta.

Experimental dependences of dissociation yield on laser pulse fluence and its frequency (or on the frequencies in two-frequency experiments) are discussed in Section 7.1. These dependences are closely related to the absorbed energy, and in the discussion the data on MP absorption is used.

The questions concerned with experimental study of dissociation products and their energy distribution are discussed in Section 7.2. The qualitative interpretation of these experiments is usually based on the statistical theory of unimolecular reactions.

In Section 7.3 we consider one more possibility of unimolecular transformation of vibrationally excited molecules, that is the process of isomerization.

In the end of this chapter, in Section 7.4, we think it useful to consider the questions connected with collisional processes. The influence of collisions on molecular phototransformations may be very essential. Their inclusion is often necessary for the correct interpretation of the data obtained in an experiment.

\subsection{Characteristics of IR multiple photon dissociation}

Dissociation in a sufficiently strong IR field has been observed in a large number of polyatomic molecules with the laser radiation frequency tuned to the absorption band active in IR absorption. For some molecules this effect also took place as the excitation was done through the overtones or combination modes. Table 7.1 list(s) molecules in which the effect of MP dissociation has been observed.

The basic parameters which characterize quantitatively the process of MP dissociation are dissociation yield $\beta$ and its quantum efficiency $\varphi=\beta D / \bar{\varepsilon}(\bar{\varepsilon}$ is the average value of absorbed energy, $D$ is the bond breaking energy). The dissociation yield is defined as a fraction of molecules dissociating per laser pulse on the gas volume under irradiation. In many works consideration has been given to the dependence of $\beta$ and $\varphi$ on laser radiation frequency $\Omega$, pulse energy fluence $\Phi$, energy absorbed by a molecule $\bar{\varepsilon}$, etc. The effect of MP dissociation for the molecules acted upon with two successive IR pulses of different 
TABLE 7.1

The molecules, for which the effect of MP dissociation has been observed

\begin{tabular}{|c|c|}
\hline $\begin{array}{l}\text { 4-atomics: } \\
\mathrm{BCl}_{3}{ }_{2,87,105-108} \\
\mathrm{D}_{2} \mathrm{CO}^{109} \\
\mathrm{H}_{2} \mathrm{CO}^{110} \\
\mathrm{HDCO}^{109-111} \\
\mathrm{DN}_{3}{ }_{112} \\
\mathrm{HN}_{3} 112\end{array}$ & $\begin{array}{l}\mathrm{SeF}_{6}{ }^{143} \\
\mathrm{MoF}_{6}{ }^{144} \\
\mathrm{UF}_{6}{ }_{145-147} \\
\mathrm{CH}_{3} \mathrm{NO}_{2}{ }^{6,25,148} \\
\mathrm{CH}_{3} \mathrm{NH}_{2}{ }_{6,43,127} \\
\mathrm{CH}_{3} \mathrm{ONO}^{149} \\
\mathrm{CH}_{2} \mathrm{CHCN}^{60,102}\end{array}$ \\
\hline 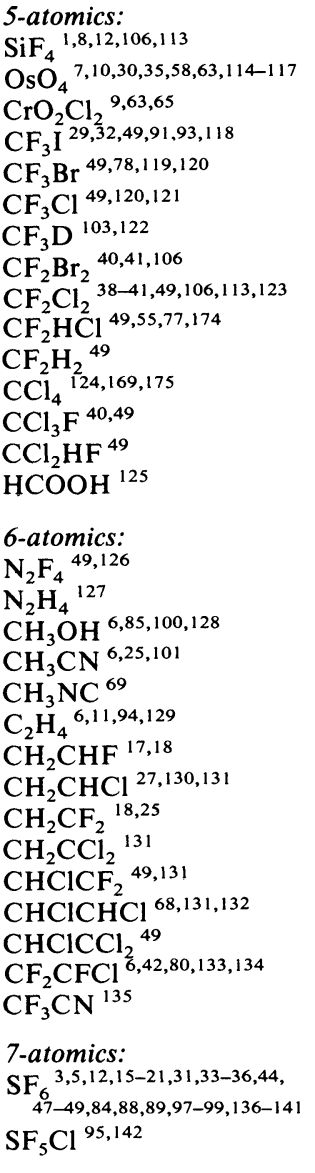 & 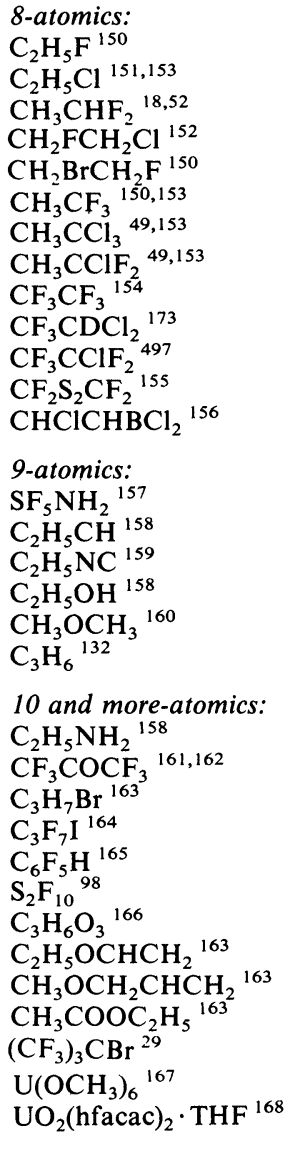 \\
\hline
\end{tabular}


frequencies has also been studied. The characteristics of the process considered below have been taken from a large number of specific typical cases. When choosing cases we took into consideration the completeness of the studies of a particular molecule. The $\mathrm{SF}_{6}$ molecule is beyond competition here since it has been studied in several dozens of works. The molecules $\mathrm{OsO}_{4}, \mathrm{CF}_{3} \mathrm{I}, \mathrm{SiF}_{4}$ have been also covered completely enough. In some cases, however, we shall be using the results on some molecules studied in single works.

Let us begin with a short review of the basic methods of study of molecular photodissociation by IR radiation.

\subsubsection{Experimental methods of IR multiple photon dissociation investigation}

The simplest method of measuring the dissociation yield $\beta$ is to measure the decrease of initial substance amount in the cell after irradiation by recording, for example, IR absorption spectra. ${ }^{3}$ In this case the change in the linear absorption coefficient $\kappa$ is related to $\beta$ by

$$
\kappa=\kappa_{0}(1-\beta \Gamma)^{n}
$$

or at small yields $(\beta \Gamma \ll 1)$ by

$$
\kappa=\kappa_{0} \exp (-n \beta \Gamma)
$$

where $\kappa_{0}$ is the absorption coefficient before irradiation, $n$ is the number of irradiation pulses, $\Gamma$ is the ratio of the exposed volume to the total volume of the cell.

The determination of the dissociation yield directly from the consumption of the primary species correctly reflects the result of IR pulse action only in the cases when we may neglect the reverse process of fragment recombination. The search for a suitable radical acceptor for each molecule is an independent problem and, as a rule, acceptors are usually found. This point will be studied in more detail in Section 7.4. Nevertheless, even though it is very simple to measure dissociation yields by monitoring the primary species, much attention has been given to the development of other direct and indirect methods which allow the elimination of secondary processes.

The most universal of these methods is based on a molecular beam technique. ${ }^{4,5}$ In this technique (see Figure 7.1) the laser beam crosses the molecular beam and dissociates the molecules. Even if the 


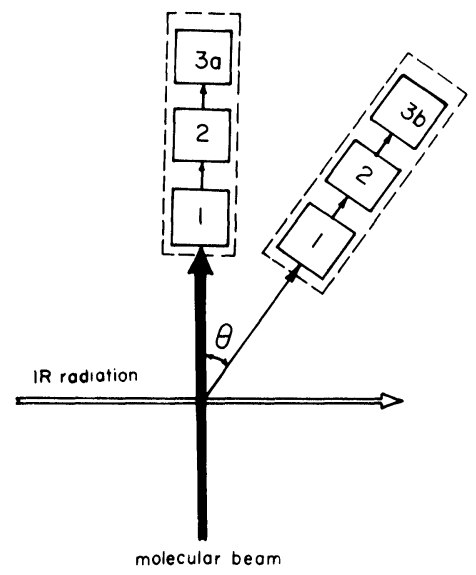

FIGURE 7.1 Schematic diagram of the molecular beam method for observing the effect of MP dissociation. (a) The dissociation yield is measured from the decrease of molecular density in the beam. (b) Angular distribution of radicals is measured. Diagram elements: (1) ionization chamber, (2) quadrapole mass filter, (3a) ion-counting system, ${ }^{97}$ (3b) multiplier. ${ }^{5}$

molecular energy exceeds the dissociation energy just by one IR photon the radicals formed must have an energy that would be much higher than the translational energy of a molecule in the beam. A part of this energy is transferred to translational degrees of freedom, and by virtue of conservation laws the radicals must acquire a momentum component in a transverse direction. Thus, the radicals can be spatially separated from the molecules. Dissociation yield can be measured both from the decrease of number of molecules in the beam itself and by recording the radicals leaving the beam. Detection is carried out with an ionization mass spectrometer which enables the arising fragments to be identified. An important advantage of the method is that it is possible to measure the angular distribution of radicals by changing the angle between the beam direction and the mass spectrometer axis. From the angular distribution function we can determine the translational energy distribution of the fragments. ${ }^{4}$

The methods dependent on detection of the visible luminescence which often follows MP dissociation are less universal but nevertheless in some cases are very effective (see Chapter 1 and below Section 7.2). It should be noted that it is just because of this phenomenon that the 
effect of collisionless MP dissociation was revealed in the very first works. ${ }^{1,2}$ The luminescence source may be radicals if they are formed in the electronically excited state. ${ }^{1,6}$ In a number of cases ${ }^{7-9}$ luminescence is from the molecules themselves. Visible luminescence is convenient for measurements since it can be easily detected. By studying the luminescence quenching on the walls (time-of-flight spectroscopy) it is possible in some cases to determine the mass of emitting particles. ${ }^{10}$ Before using visible luminescence to measure the dissociation yield, however, their direct proportional dependence must be determined. In some cases, for example in Refs. 11 and 12, it has been proven that this requirement is fulfilled.

When it is known that in the process of MP dissociation radicals with line absorption spectra in the visible or near UV regions are formed, they can be recorded using the laser induced fluorescence (LIF) method. ${ }^{13,61}$ The typical scheme of the LIF experiment is shown in the Figure 7.2. In this method excitation of the radicals from the ground state is performed by a tunable dye laser, and the fluorescence of electronically-excited radicals thus induced is measured. An important advantage of this method is its high sensitivity which enables radical concentrations from $10^{8}$ to $10^{9} \mathrm{~cm}^{-3}$ to be detected.

One more possibility of studying the process of MP dissociation is connected with recording the chemiluminescence signal. This method

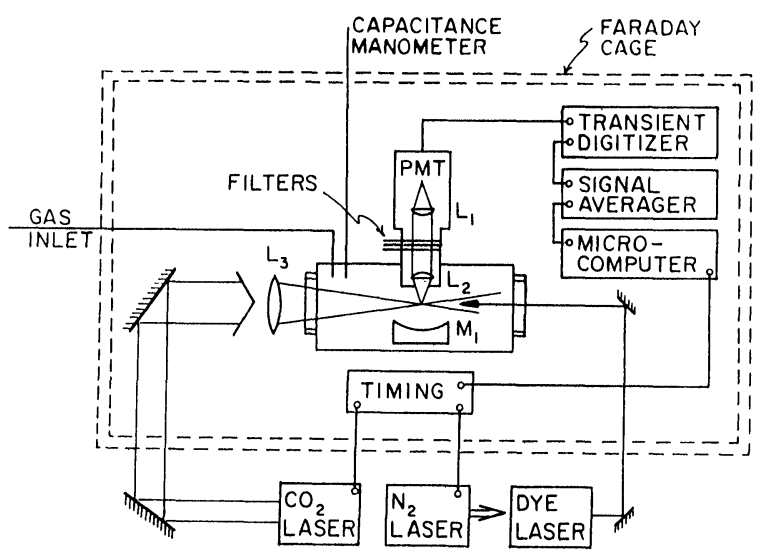

FIGURE 7.2 Scheme of experiment to detect radicals by the method of laser induced fluorescense. $L_{1}, L_{2}, L_{3}$ and $M_{3}$ are lenses and a mirror. (From Ref. 61.) 
was used ${ }^{14}$ when the isotopic selectivity of MP dissociation was first discovered. In this work the mixture $\mathrm{BCl}_{3}+\mathrm{O}_{2}$ was irradiated and the chemiluminescence of $\mathrm{BO}^{*}$ radicals in the visible region was observed. A modification of this method was used ${ }^{15,16}$ to detect $F$ atoms. They were recorded by the IR fluorescence of the vibrationally-excited HF* molecules formed by the fast reaction $\mathrm{F}+\mathrm{H}_{2} \rightarrow \mathrm{HF}^{*}+\mathrm{H}$.

An interesting study has been ${ }^{17,18}$ the MP dissociation of mixed halogenated ethanes and ethylenes. In this case, by unimolecular dissociation, vibrationally-excited $\mathrm{HF}^{*}$ molecules are formed which allow them to be detected by IR fluorescence.

All the above-listed methods were used to demonstrate molecular photodissociation as well as to measure the dependence of dissociation yield $\beta$ on laser pulse energy fluence $\Phi$ and laser radiation frequency $\Omega$. The molecular beam method and the laser induced fluorescence method have obtained in some cases direct information on dissociation products and their energy distribution. These results will be discussed in the succeeding sections.

In conclusion it should be noted that the probing of radicals with Raman scattering may turn out to be one more promising method for studying the process of MP dissociation. In this case it would be possible to measure both the radical concentration and their internal energy. ${ }^{96}$

\subsubsection{Dependence of MP dissociation yield and quantum efficiency on laser pulse energy fluence and absorbed energy}

Dissociation threshold. Even in the first works, ${ }^{1,2}$ on MP molecular dissociation, where the visible luminescence of molecules in strong IR fields was studied, particular attention was concentrated on a fast dependence of luminescence signal on laser pulse intensity. This property was treated, as usual, as the presence of a threshold for the effect observed.

The threshold properties of the effect of MP dissociation were first studied in detail ${ }^{19}$ for the $\mathrm{SF}_{6}$ molecule. The results of this work have been discussed already in Chapter 4 (see Section 4.4.1).

The threshold properties of MP dissociation were also studied in other works both for the $\mathrm{SF}_{6}$ molecule and other molecules. Some results are presented in Table 7.2. It should be noted, however, that the data presented are conventional in character and the very concept 
TABLE 7.2

Threshold values of fluence for MP dissociation of some molecules

\begin{tabular}{lcl}
\hline \multicolumn{1}{c}{ Molecule } & $\Phi_{\mathrm{th}}{ }^{\mathrm{a}}, \mathrm{J} \cdot \mathrm{cm}^{-2}$ & \multicolumn{1}{c}{ References and comments } \\
\hline $\mathrm{BCl}_{3}$ & 20 & 2,105 \\
$\mathrm{SiF}_{4}$ & 4.5 & for $\nu_{3}$ mode excitation, 113 \\
& 15 & for $\nu_{1}+2 \nu_{2}-\nu_{4}$ excitation, 1 \\
$\mathrm{~N}_{2} \mathrm{H}_{4}$ & 0.1 & 127 \\
$\mathrm{OsO}_{4}$ & $<0.2$ & The threshold is dependent on laser frequency, 30 \\
$\mathrm{SF}_{6}$ & $0.5-5$ & $5,18,19,47,84,113,136-138$. The threshold is \\
& & dependent on laser frequency, 137, 138 \\
$\mathrm{SF}_{5} \mathrm{Cl}$ & 0.7 & 95 \\
$\mathrm{SF}_{5} \mathrm{NF}_{2}$ & $<10^{-2}$ & 157 \\
$\mathrm{~S}_{2} \mathrm{~F}_{10}$ & $<10^{-2}$ & 98 \\
$\mathrm{CF}_{3} \mathrm{D}$ & $<10$ & 103 \\
$\mathrm{CF}_{3} \mathrm{Br}$ & $<1.0$ & 119 \\
$\mathrm{CF}_{3} \mathrm{I}$ & $<0.3$ & 32 \\
$\mathrm{CCl}_{4}$ & 1.2 & for $\nu_{3}$ mode excitation, 169 \\
& 120 & for $\nu_{1}+\nu_{2}+\nu_{4}$ excitation, 169 \\
$\mathrm{CH}_{3} \mathrm{CN}$ & 60 & The threshold is dependent on radiation intensity \\
& & $I_{\text {th }} \approx 10^{9} \mathrm{~W}$ cm \\
& 11,25 \\
$\mathrm{CH}_{3} \mathrm{OH}$ & $10-20$ & 100 \\
$\mathrm{C}_{2} \mathrm{H}_{4}$ & 2 & 170 \\
$\mathrm{C}_{2} \mathrm{H}_{3} \mathrm{~F}$ & $20-40$ & 130 \\
$\mathrm{C}_{2} \mathrm{H}_{3} \mathrm{Cl}$ & 0.7 & 162 \\
$\mathrm{CF}_{3} \mathrm{COCF}$ & & 130 \\
\hline
\end{tabular}

${ }^{a}$ The threshold was estimated when its value was not given by authors.

of a threshold depends on the sensitivity of the method we use to detect the dissociation products. Some appropriate studies have shown that near the threshold the dependence $\beta=\beta(\Phi)$ is steep but continuous. The value of $\beta$ drops quickly to $10^{-4}-10^{-5}$ with an insignificant decrease of $\Phi$ in a region below "the threshold" which is understood as the existence of some threshold for MP dissociation. Even though the term "dissociation threshold" is used, one should keep in mind its conventional nature.

Some differences in determining the threshold value, particularly for $\mathrm{SF}_{6}$, may be caused by different experimental conditions. For example, it has been demonstrated [7.20,7.21] the necessity of a correct determination of the spatial energy distribution across the laser beam. The geometric factor is of particular importance when the laser radiation is focused. ${ }^{22-24}$

We have discussed earlier in Chapter 4 (see Section 4.4.1) the physical nature of the MP dissociation threshold. In most cases the 
threshold of MP dissociation is caused by excitation of the molecules through the region of vibrational quasi-continuum. The energy distribution function formed as a result of this excitation has a sharp exponential tail in the region of high energies. The position of this tail becomes higher as the laser pulse fluence increases, and the dissociation threshold is apparently related physically to the value of $\Phi_{\text {th }}$ when the distribution tail reaches the dissociation limit. Such an explanation of threshold is generally used for molecules whose quasicontinuum limit is low enough, and therefore they dissociate at rather moderate pulse energy fluences $\left(1\right.$ to $\left.10^{2} \mathrm{~J} / \mathrm{cm}^{2}\right)$.

MP dissociation for molecules which call for high energy fluences $\left(\geqslant 10^{2} \mathrm{~J} / \mathrm{cm}^{2}\right)$ is not so unambiguous. It has been pointed out that radiation intensity is essential in MP dissociation of vinyl chloride, ethyl vinyl ether and acetone nitrile. ${ }^{25-27}$

Dependence of $\beta$ and $\varphi$ on laser energy fluence and absorbed energy. As the energy fluence exceeds the threshold the dissociation yield increases rapidly. When the value of $\beta$ becomes significant, more than approximately fifteen per cent, the increase of the dependence slows down and saturation begins. Usually as the energy fluence is increased by about an order as compared to its threshold value the saturation of the dependence becomes appreciable. Some characteristic examples of such dependences are given in Figure 7.3.

MP dissociation is apparently an extension of the effect of molecular MP excitation with IR radiation considered in detail in the foregoing chapters. Among other things it has been shown there that in the process of MP excitation two molecular ensembles are often formed: molecules excited to the quasi-continuum with their fraction $q$ and molecules left on the lower levels. So the character of the dependence of $\beta$ on $\Phi$ (Figure 7.3) is affected by two factors: variation of the fraction of excited molecules $q$ and variation of the average energy of excited molecules $\bar{\varepsilon}_{q}$.

Physically it is more justified to describe the process of molecular dissociation with the value of quantum dissociation efficiency $\varphi=$ $\beta(D / \bar{\varepsilon})$ that, as noted in Chapter 6, is independent of the $q$ factor. The dependence of $\varphi$ on $\Phi$ for the $\mathrm{CF}_{3} \mathrm{I}$ molecule is given in Figure 4.10. In essence, the quantum efficiency of MP dissociation shows what part of the energy absorbed in the gas is consumed to break the molecular bonds. For $\mathrm{CF}_{3} \mathrm{I}$, for example, the dissociation yield being 


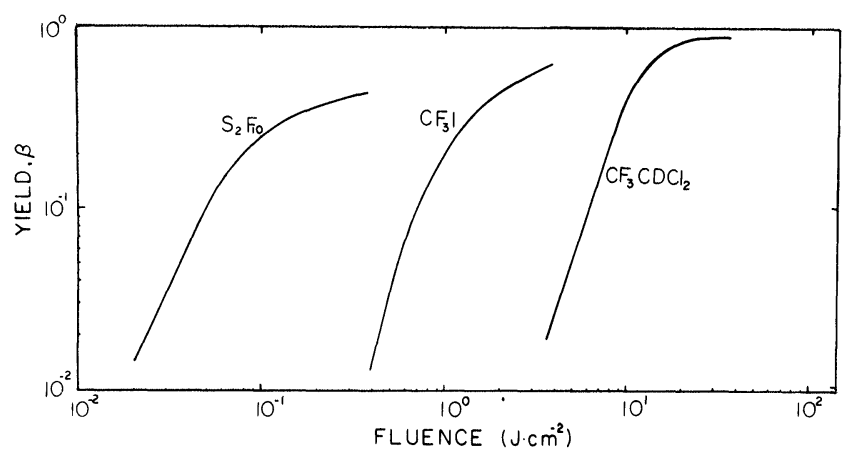

FIGURE 7.3 Characteristic dependences $\beta=\beta(\Phi)$ for different molecules (no account taken of the possible recombination). (From Refs. 98, 32, 173.)

unity, $\varphi \simeq 0.9$. The fact that $\varphi$ differs from unity is caused by overexcitation of the molecules beyond the dissociation limit. In the case of $\mathrm{CF}_{3} \mathrm{I}$ the value of overexcitation $\bar{\varepsilon}_{q}-D \simeq 1700 \mathrm{~cm}^{-1}$. The overexcitation level is determined by the ratio of the rate of laser excitation of the molecules and the rate of their dissociation. As it follows from the RRKM theory, for small (five or six-atom) molecules the dissociation rate becomes rather high even with a small excess (0.1-0.2 D) over the dissociation limit. Therefore, at the typical laser pulse duration $\tau_{p} \simeq 10^{-7} \mathrm{~s}$ no further molecular overexcitation takes place. The situation is different for large molecules (over 10-12 atoms). Much higher overexcitation is possible here. In the experiments with $\left(\mathrm{CF}_{3}\right)_{3} \mathrm{CI}$, for example, $\bar{\varepsilon}_{q} \geqslant 2 \mathrm{D}$ was observed. So the value of $\varphi$ for these molecules cannot be higher than 0.5 .

An intimate relation between dissociation yield and absorbed energy has been found..$^{28}$ In this work it was assumed that in the process of MP excitation a Boltzmann distribution is formed, its average energy determined by the energy absorbed by the molecules. It was also assumed that dissociation would take place only for the molecules having energy above the dissociation limit. Although in this work the gas temperature was not calculated quite correctly, the experimental and calculated curves for $\mathrm{SF}_{6}$ are in qualitative agreement. A similar approach within the limits of such a thermal model has been used in. $^{29,21}$ The dissociation yield here is calculated in the form

$$
\beta=Z_{\mathrm{vib}}^{-1} \int_{D}^{\infty} \rho(E) e^{-E / k T} d E
$$


where $Z_{\text {vib }}$ is the vibrational statistical sum and $\rho(E)$ is the vibrational state density. Correct calculation of temperature from the values of absorbed energy as well as inclusion of additional absorption by $\mathrm{SF}_{5}$ radicals formed in the dissociation of $\mathrm{SF}_{6}$ made it possible to consider ${ }^{21}$ quantitative (within 50\%) agreement between the calculated and measured values of dissociation yield. As one would expect, the maximum inconsistency occurs with $\beta \ll 1$ when the Boltzmann distribution tail must give (Figure 6.13) an excessive value of the yield.

Such agreement is due to the fact that, even though the energy distribution function formed in the process of MP excitation is not the Boltzmann one (see Chapter 6), its difference from the Boltzmann function for excited molecules is not cardinal. Therefore, in estimations we may use a simple thermal model. In this case, however, we should take into account one important factor, that is, a possibility of the separation of the molecules into two ensembles. Here account must be taken only of the excited molecules, and expression (7.2) can be used only for them. Otherwise the error may be as great as several orders. This can be clearly seen from Figure 6.12 when the calculated data within the frameworks of thermal model (curve 3 ) is compared with the experimental ones in two cases when the measured parameters are averaged over all molecules (curve 1) or are concerned only with the upper ensemble (curve 2).

\subsubsection{Dependence of dissociation yield on IR laser frequency}

The relationship between the effects of MP dissociation and absorption vividly manifests itself in the similarity of the spectral dependences of dissociation yield and absorbed energy. Figure 7.4a shows the dependence of dissociation yield on laser radiation frequency as the $\mathrm{SF}_{6}$ molecule is excited within the limits of the $\nu_{3}$ band. ${ }^{19}$ The maximum of this dependence like that of the corresponding dependence of absorbed energy (see Figure 4.4a) is shifted towards the long-wave edge of the spectrum, and the spectral curves, as a whole, are very alike. More striking consistency of the spectral characteristics of the two effects follows from the spectral dependence of the dissociation yield of $\mathrm{OsO}_{4}$ molecules ${ }^{30}$ presented in Figure 7.41. The structure observed is consistent with the structure in the MP absorption spectrum of this molecule (see Figure 4.4b). 

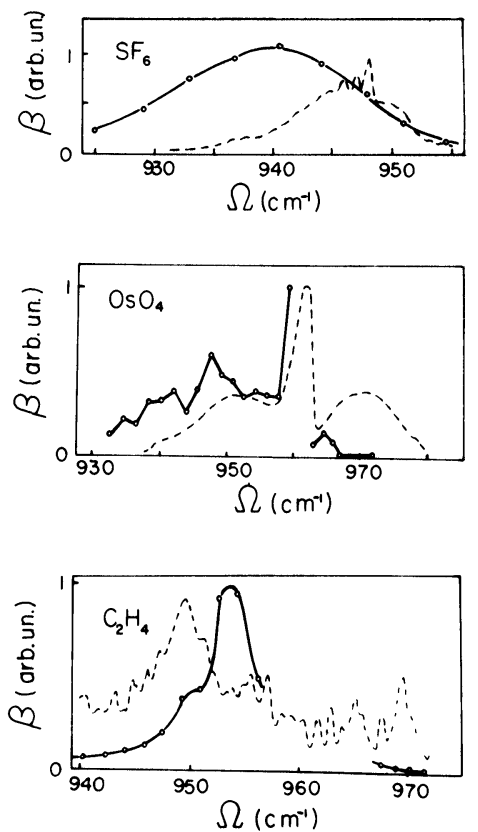

FIGURE 7.4 Dependence of the dissociation yield $\beta$ on the radiation frequency for some molecules. (a) $\mathrm{SF}_{6},{ }^{19}$ (b) $\mathrm{OsO}_{4},{ }^{30}$ (c) $\mathrm{C}_{2} \mathrm{H}_{4} \cdot{ }^{11}$ The corresponding spectra of linear absorption are shown with dashed curves.

The deformation of the MP excitation spectrum caused by changes in the excitation conditions usually brings about corresponding changes in the spectral characteristics of MP dissociation. This may be illustrated by experiments with gas cooling. The narrowing of the MP excitation spectrum caused by the temperature drop brings about a corresponding decrease in the width of dissociation yield spectral curve. $^{31}$ (See Chapter 8 about the influence of gas temperature on dissociation yield.)

It should be pointed out, however, that the consistency between the spectral dependences of absorbed energy and dissociation yield is empirical by nature. There are disparities, and the characteristic example for this is $\mathrm{C}_{2} \mathrm{H}_{4}$. In the MP dissociation spectrum of this molecule (see Figure 7.4c) there is only one maximum manifesting itself clearly, whereas there are three maxima in the MP absorption spectrum. ${ }^{11}$ 
Localization of the MP dissociation spectrum near the linear absorption spectrum of the corresponding vibration of the molecule under excitation (see Figure 7.4a, b, c) makes possible selective dissociation of molecules in a mixture, for example containing a certain isotope. Although the MP dissociation spectrum, like the MP absorption spectrum, is usually wider than the linear absorption spectrum, its width in many cases, nevertheless, is smaller than the isotope shift between the absorption bands of different isotopic molecules. The isotopic selectivity of MP dissociation has found wide application in isotope separation and in other selective processes. The data on this point are given in Chapters 8 and 9.

Let us discuss one more aspect of the relationship between the characteristics of MP dissociation and the exciting radiation frequency. The dependence of dissociation yield on laser radiation frequency causes the dependence $\beta=\beta(\Phi)$ to change with changes in $\Omega$. This can be clearly seen from Figure 7.5a which presents the dependences of $\beta$ on $\Phi$ as $\mathrm{SF}_{6}$ is excited at two different $\mathrm{CO}_{2}$ laser lines. A natural question arises whether the dependence of dissociation yield on absorbed energy changes as the radiation frequency varies. In other words, whether the dissociation yield depends only on absorbed energy or with the same $\bar{\varepsilon}$, it also depends on radiation frequency. This question has been studied. ${ }^{21}$ Figure $7.5 \mathrm{~b}$ gives the values of MP dissociation yield for $\mathrm{SF}_{6}$ varying with the value of absorbed energy
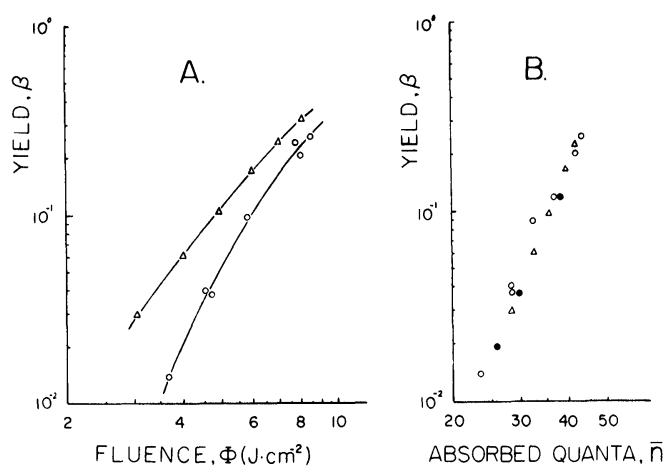

FIGURE 7.5 MP dissociation of $\mathrm{SF}_{6}{ }^{2}$ (a) Dependence of $\beta$ on $\Phi$ at the $P(16)$ and $P(24)$ lines of $\mathrm{CO}_{2}$ laser. (b) Dependence of $\beta$ on the number of absorbed quanta for the $P(16), P(24)$ and $P(28)$ lines of $\mathrm{CO}_{2}$ laser. $\bigcirc P(26) ; \triangle P(24) ; \bigcirc P(28)$. 
during excitation at three different $\mathrm{CO}_{2}$ laser frequencies. It may be seen that the values of $\beta$ at all three lines coincide for the same values of the number of absorbed quanta $n$ within the experimental limits.

Thus, in the case of $\mathrm{SF}_{6}$ the dissociation yield is actually determined only by the value of absorbed energy, at least when excitation takes place within the linear absorption band $\nu_{3}$. This fact enabled the authors ${ }^{21}$ to conclude that the vibrational distribution function, too, does not apparently depend on excitation frequency and is fully determined by the average energy of the excited molecules $\bar{\varepsilon}$. A similar assumption on the universal character of the distribution function in the MP excitation of $\mathrm{CF}_{3} \mathrm{I}$ had been made before. ${ }^{32}$ It is consistent with the experimental measurement of the MP dissociation parameters of this molecule.

It is quite possible that in MP excitation of molecules, at least at not very large shifts from the center of the linear absorption band of the vibration chosen, the form of the vibrational distribution function really depends a little on radiation frequency. The data available is not enough, however, for unambiguous conclusions. In measurements of dissociation yield the results may be affected by the collisional equilibration towards a Boltzmann description of the distribution function caused by collisions after MP excitation and before a major number of excited molecules has dissociated. The question on the universality of the distribution function remains to be solved. Only direct measurements of the form of the function of vibrational distribution of excited molecules can apparently provide the answer.

\subsubsection{MP dissociation in two-frequency IR field}

According to the presently accepted theory, there are two main stages in the process of MP excitation: (1) excitation through the lower vibrational levels and (2) subsequent absorption of energy in the vibrational quasi-continuum. This is confirmed by experiments on two-frequency irradiation of molecules. The first experiment of this kind was published in $1976 .^{33}$ The idea of two-frequency irradiation is that the function of the radiation for the first and second stages of the process may be different and can be divided. First the molecule is acted upon by the first pulse with a $\Omega_{1}$ frequency within the absorption band. The second pulse with a different frequency $\Omega_{2}$ provides subsequent excitation of the molecules in the quasi- 
continuum. This frequency can be even brought out beyond the MP absorption contour observed for one-frequency irradiation.

The first work ${ }^{33}$ revealed such an important property of the effect of two-frequency dissociation as the absence of a threshold for the energy fluence of the first pulse. The dependence $\beta\left(\Phi_{1}\right)$ measured $^{34}$ is shown in Figure 7.6. The $\mathrm{SF}_{6}$ molecule was excited at the frequency $\Omega_{1}=942.4 \mathrm{~cm}^{-1}$, that corresponded approximately to the maximum of multiphoton IR absorption, and $\Omega_{2}=954.5 \mathrm{~cm}^{-1}$. The energy fluence of the second pulse was constant $\Phi_{2}=7.5 \mathrm{~J} / \mathrm{cm}^{2}$. It may be seen that

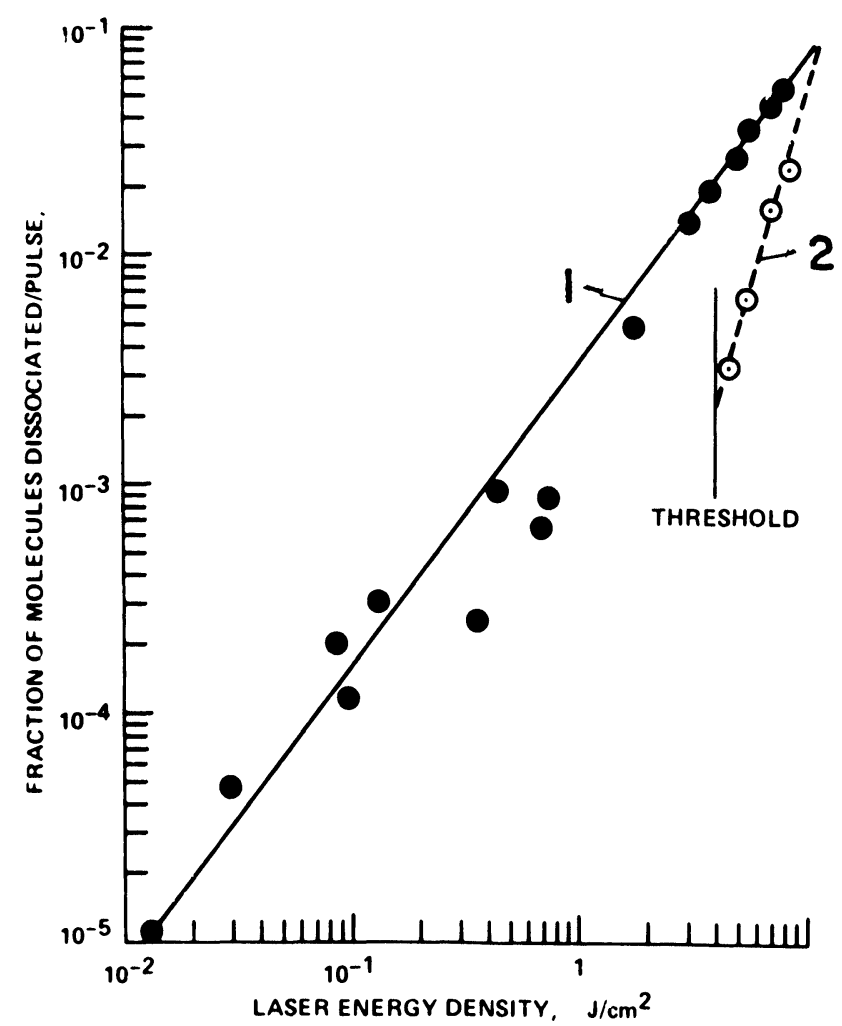

FIGURE 7.6 Two-frequency MP dissociation of $\mathrm{SF}_{6}$ molecules. ${ }^{34}$ Dependence $\beta=$ $\beta\left(\Phi_{1}\right)$ with excitation performed by the first field at the frequency $\Omega_{1}=942.4 \mathrm{~cm}^{-1}$ in the presence of the second field at $\Omega_{2}=945.5 \mathrm{~cm}^{-1}$ with the second pulse fluence $\Phi_{2}=7.5 \mathrm{~J} / \mathrm{cm}^{2}$ (curve 1). The dependence $\beta=\beta\left(\Phi_{2}\right)$ in the absence of the second pulse (curve 2) is given for comparison. 
the dissociation yield increases almost linearly as $\Phi_{1}$ increases. This dependence differs greatly from that dependence measured in the absence of the second pulse which has a threshold character. This result shows once again that the interpretation that the threshold property of the MP dissociation effect is related to molecular excitation in the vibrational quasi-continuum is correct.

The studies of the frequency dependences $\beta=\beta\left(\Omega_{1}, \Omega_{2}\right)^{33,12,35}$ have shown that the resonant character of MP dissociation is basically related to the passage through the lower vibrational levels. As far as the transitions in the vibrational quasi-continuum are concerned, their character is also resonant, although it is much weaker. This conclusion follows from measuring the dependence $\beta=\beta\left(\Omega_{2}\right)$ with $\Omega_{1}=$ const in experiments on such molecules as $\mathrm{OsO}_{4},{ }^{35} \mathrm{SiF}_{4}{ }^{12} \mathrm{SF}_{6}{ }^{12,34,36}$ In all these cases the spectral dependence $\beta=\beta\left(\Omega_{2}\right)$ is shifted considerably to the red spectral region (see Figure 6.7). The results obtained enable us to conclude that the vibrational quasi-continuum in the vicinity of the normal vibration under consideration is a wide resonance shifted towards the low-frequency region because of vibration anharmonicity. The spectral properties of the quasi-continuum are discussed in more detail in Chapter 6.

Optimalizing conditions for molecular excitation in the quasicontinuum, as one laser pulse is divided into two pulses and one of them is shifted towards the long-wave region, has two important consequences. First, the MP dissociation threshold is reduced. This can be seen from comparing the dependences $\beta=\beta\left(\Phi_{2}\right)$ for different frequencies $\Omega_{2}$ of the second pulse presented in Figure 7.7. Second, the use of two-frequency excitation above the dissociation threshold may result in a considerable (about an order and more) increase in yield $\beta$ with the total energy of two pulses being constant. The value of this increase in $\beta$ depends on the second pulse frequency $\Omega_{2}$, which can be clearly seen from Figures 6.7 and 7.7, and on radiation energy fluence when $\Omega_{1}$ and $\Omega_{2}$ are fixed. The latter follows from the data presented in Figure 7.8. The dependence of the dissociation yield of $\mathrm{SF}_{6}$ on energy fluence $\Phi_{2}$ here is given for several fixed values of $\Phi_{1}$. It may be seen that even a small addition of radiation at the $\Omega_{1}$ frequency causes an appreciable increase in yield as compared to one-frequency excitation. If we reconstruct curves 2,3 and 4 in Figure 7.8 as the function of the total energy fluence in both fields $\Phi_{1}+\Phi_{2}$ they fall on the same curve (the dashed one). If we compare the latter 


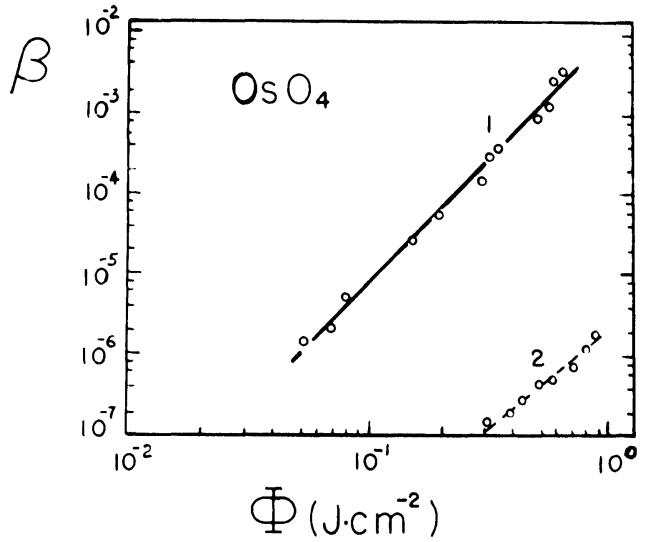

FIGURE 7.7 Two-frequency MP dissociation of $\mathrm{OsO}_{4}$ molecules. ${ }^{35}$ The dependences $\beta=\beta\left(\Phi_{2}\right)$ for two different frequencies of the second field: $\Omega_{2}=929.02 \mathrm{~cm}^{-1}$ (curve 1) and $\Omega_{2}=966.25 \mathrm{~cm}^{-1}$ (curve 2). In the both cases $\Omega_{1}=954.55 \mathrm{~cm}^{-1}, \Phi_{2}=0.2 \mathrm{~J} / \mathrm{cm}^{2}$.

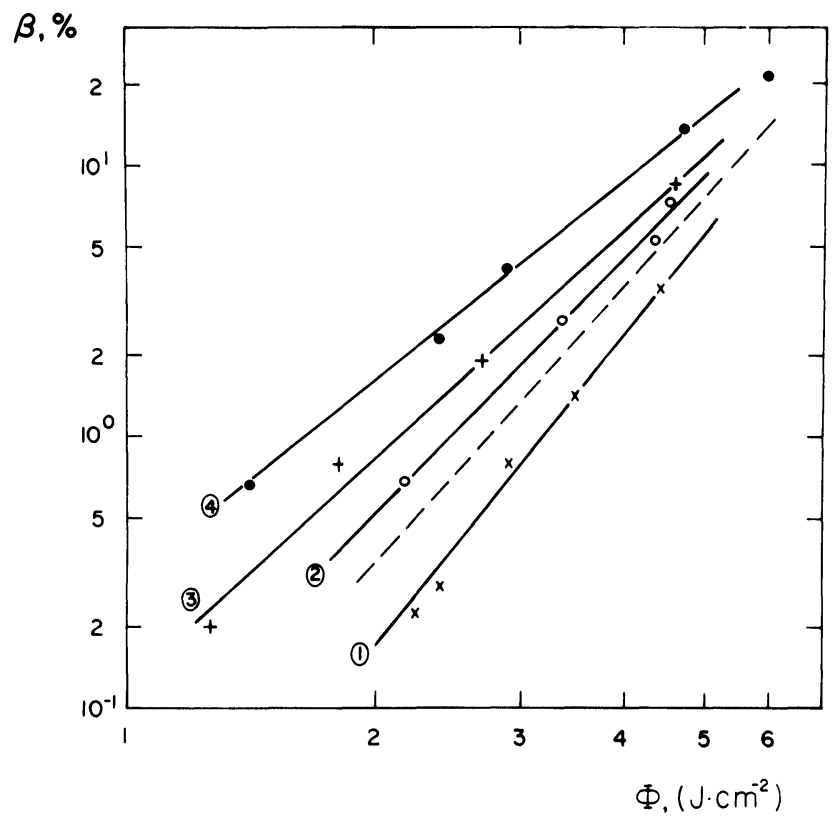

FIGURE 7.8 Dependence of the dissociation yield of $\mathrm{SF}_{6}$ on fluence $\Phi=\Phi_{1}+\Phi_{2}$ (dashed line) and $\Phi=\Phi_{2}$ (solid lines) with $\Phi_{1}=0(1) ; 0.23(2), 0.57(3)$ and $1.1(4) \mathrm{J} / \mathrm{cm}^{2} .{ }^{99}$ 
with curve 1 we can determine the pure gain obtained from dividing the energy of the $\Phi_{2}$ field into twc parts. In the region of low energy fluence $\left(\sim 2 \mathrm{~J} / \mathrm{cm}^{2}\right)$ the gain in the two-frequency method, no matter in which way the field is divided, comes to about $100 \%$. As the energy fluence increases the gain becomes decreased.

Besides the possibility of increasing the dissociation yield, the two-frequency method has one more property important in practice. When the energy fluence of the first pulse which is responsible for resonant excitation of molecules through the lower levels is reduced this causes the MP excitation spectrum to get narrower (see Chapter 5 ) and, as a result, the dissociation selectivity of the desired molecules to increase. This property of two-frequency dissociation has found application in laser isotope separation (see Chapter 8).

\subsection{Products of IR multiple photon dissociation and their energy distribution}

In this section we are going to consider the products formed by the process of molecular MP dissociation as well as their energy distribution. We can use several methods to determine the primary dissociation products. In some cases they can be found from the final stable dissociation products if we know the secondary chemical reactions. In most cases, however, indirect determination of primary fragments cannot replace the direct diagnostic methods. The largest progress can be achieved with the use of the molecular beam technique as well as the laser induced fluorescence of radicals technique. These methods enable us not only to identify directly the primary dissociation fragments but they also give important information on the form of the energy distribution of these products.

In discussing the results stated in this section we shall widely use the statistical theory of unimolecular reactions (RRKM). This theory forms the basis for understanding the process of dissociation of the vibrationally excited molecules as a result of their interaction with the IR radiation. All necessary information from the RRKM theory can be found in Section 2.4.

\subsubsection{MP dissociation channels and primary fragments}

Table 7.3 presents data on products of molecular MP dissociation by IR radiation. This data was obtained in some cases from indirect 
considerations but in most cases the products formed were detected using direct diagnostic methods.

In the second column of Table 7.3 there are main dissociation channels observed in the process of MP excitation of molecules. The data presented shows that the resulting products, as a rule, correspond to the breaking of the weakest molecular bond.

In molecules with two close dissociation channels dissociation is possible by both channels. This can be illustrated with the $\mathrm{C}_{2} \mathrm{H}_{4} \mathrm{FCl}$ molecule which has the following dissociation channels.

$$
\begin{array}{ll}
\mathrm{C}_{2} \mathrm{H}_{4} \mathrm{FCl}{ }_{k(2)}^{k(1)} \mathrm{C}_{2} \mathrm{H}_{3} \mathrm{Cl}+\mathrm{HF} & \left(E_{A}=21260 \mathrm{~cm}^{-1}\right) \\
\mathrm{C}_{2} \mathrm{H}_{3} \mathrm{~F}+\mathrm{HCl} & \left(E_{A}=22300 \mathrm{~cm}^{-1}\right)
\end{array}
$$

( $E_{A}$ is the activation energy). The dissociation rates ratio estimated ${ }^{37}$ according to the RRKM theory is in good agreement with the concentration ratio of $\mathrm{C}_{2} \mathrm{H}_{3} \mathrm{~F}$ and $\mathrm{C}_{2} \mathrm{H}_{3} \mathrm{Cl}$ measured experimentally. It should be noted that the relative contribution of different channels may depend on the radiation intensity since the dependences of the dissociation rate constants $k(1)$ and $k(2)$ on molecular excitation level may be different.

In the presence of two close dissociation channels one of which is followed by elimination of atoms and the other one by elimination of molecules the first channel often turns out to be more probable even if energetically less advantageous. We can illustrate this with the $\mathrm{CF}_{2} \mathrm{Cl}_{2}$ molecule which has the following dissociation channels

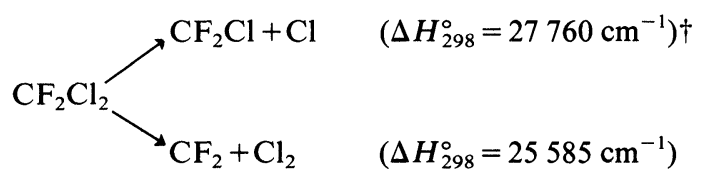

In this case the dissociation probability by the first channel is about an order higher than by the second one. ${ }^{38-40}$ Dissociation by this energetically "disadvantageous" channel has been also observed in MP excitation of $\mathrm{CF}_{2} \mathrm{Br}_{2},{ }^{41} \mathrm{CF}_{2} \mathrm{CFCl}^{43}$ and $\mathrm{CH}_{3} \mathrm{NH}_{2} \cdot{ }^{43}$ For the first

† In many cases the value of dissociation energy is determined from the thermal effect of reaction, i.e., from the difference $\Delta H^{\circ}$ in enthalpies of formation of primary and final products. The values $\Delta H_{0}^{\circ}$ and $\Delta H_{298}^{\circ}$ are given for zero and room temperatures respectively. When there is no barrier for the reverse reaction $\Delta H_{0}^{\circ}=D$. 
TABLE 7.3

Primary fragments of MP dissociation for some molecules

\begin{tabular}{|c|c|c|c|}
\hline Molecule & $\begin{array}{l}\text { Main channel } \\
\text { of MP } \\
\text { dissociation }\end{array}$ & References & Comments \\
\hline $\mathrm{NH}_{3}$ & $\mathrm{NH}_{2}+\mathrm{H}$ & 13 & \\
\hline $\mathrm{N}_{2} \mathrm{H}_{4}$ & $2 \mathrm{NH}_{2}$ & 127 & \\
\hline $\mathrm{N}_{2} \mathrm{~F}_{4}$ & $2 \mathrm{NF}_{2}$ & $40,49,126$ & \\
\hline $\mathrm{SF}_{6}$ & $\mathrm{SF}_{5}+\mathrm{F}$ & $15,16,46,48$ & $\mathrm{SF}_{5} \stackrel{n \hbar \Omega}{\longrightarrow} \mathrm{SF}_{4}+\mathrm{F}^{45,48}$ \\
\hline $\mathrm{SF}_{5} \mathrm{Cl}$ & $\mathrm{SF}_{5}+\mathrm{Cl}$ & 142 & $\mathrm{SF}_{5} \stackrel{n \hbar \Omega}{\longrightarrow} \mathrm{SF}_{4}+\mathrm{F}(?){ }^{142}$ \\
\hline $\mathrm{SF}_{5} \mathrm{NF}_{2}$ & $\mathrm{SF}_{5}+\mathrm{NF}_{2}$ & 157 & \\
\hline $\mathrm{S}_{2} \mathrm{~F}_{10}$ & $2 \mathrm{SF}_{5}$ & 98 & \\
\hline $\mathrm{CHFCl}_{2}$ & $\mathrm{CFCl}+\mathrm{HCl}$ & 49,50 & \\
\hline $\mathrm{CF}_{2} \mathrm{HCl}$ & $\mathrm{CF}_{2}+\mathrm{HCl}$ & $49,50,55,171$ & $\begin{array}{l}\text { Other channels: } \mathrm{CFCl}+\mathrm{HF} \text {, } \\
\mathrm{CHCl}+\mathrm{F}_{2}, \mathrm{CHF}+\mathrm{ClF} \text {-less } \\
\text { than } 1 \% \text { of the main } \\
\text { channel }\end{array}$ \\
\hline $\mathrm{CF}_{3} \mathrm{D}$ & $\mathrm{CF}_{2}+\mathrm{DF}$ & 103 & \\
\hline $\mathrm{CF}_{3} \mathrm{Cl}$ & $\mathrm{CF}_{3}+\mathrm{Cl}$ & 40,49 & $\mathrm{CF}_{3} \stackrel{n \hbar \Omega}{\longrightarrow} \mathrm{CF}_{2}+\mathrm{F}$ \\
\hline $\mathrm{CF}_{3} \mathrm{Br}$ & $\mathrm{CF}_{3}+\mathrm{Br}$ & 40,49 & \\
\hline $\mathrm{CF}_{3} \mathrm{I}$ & $\mathrm{CF}_{3}+\mathrm{I}$ & $40,49,93$ & \\
\hline $\mathrm{CF}_{2} \mathrm{Cl}_{2}$ & $\mathrm{CF}_{2} \mathrm{Cl}+\mathrm{Cl}$ & $38,40,49$ & $\begin{array}{l}\text { The other channel } \mathrm{CF}_{2}+ \\
\mathrm{Cl}_{2}{ }^{41}\end{array}$ \\
\hline $\mathrm{CF}_{2} \mathrm{Br}_{2}$ & $\mathrm{CF}_{2} \mathrm{Br}+\mathrm{Br}$ & 40,49 & $\begin{array}{l}\text { The other channel } \mathrm{CF}_{2}+ \\
\mathrm{Br}_{2}{ }^{41}\end{array}$ \\
\hline $\mathrm{CFCl}_{3}$ & $\mathrm{CFCl}_{3}+\mathrm{Cl}$ & 40,49 & $\mathrm{CFCl}_{2} \stackrel{n \hbar \Omega}{\longrightarrow} \mathrm{CFCl}+\mathrm{Cl}^{40}$ \\
\hline $\mathrm{C}_{2} \mathrm{H}_{4}$ & $\mathrm{H}_{2} \mathrm{CC}+\mathrm{H}_{2}(?)$ & 129,170 & $\begin{array}{l}\mathrm{H}_{2} \mathrm{CC} \stackrel{n \hbar \Omega}{\longrightarrow} \mathrm{C}_{2}+\mathrm{H}_{2}{ }^{129} \\
\text { Visible luminescence of } \mathrm{C}_{2} \\
\text { radical }^{6,129}\end{array}$ \\
\hline $\mathrm{CH}_{2} \mathrm{CHF}$ & $\mathrm{C}_{2} \mathrm{H}_{2}+\mathrm{HF}$ & 56 & \\
\hline $\mathrm{CH}_{2} \mathrm{CHCl}$ & $\mathrm{C}_{2} \mathrm{H}_{2}+\mathrm{HCl}$ & 30 & \\
\hline $\mathrm{CH}_{2} \mathrm{CF}_{2}$ & $\mathrm{C}_{2} \mathrm{HE}+\mathrm{HF}$ & 52,56 & \\
\hline $\mathrm{CHClCCl}_{2}$ & $\mathrm{C}_{2} \mathrm{HCl}_{2}+\mathrm{Cl}$ & 49 & \\
\hline $\mathrm{CF}_{2} \mathrm{CFCl}$ & $\longrightarrow \mathrm{CF}_{2}+\mathrm{CFCl}$ & $\begin{array}{l}42 \\
133\end{array}$ & $\begin{array}{l}\text { Visible luminescence of } \mathrm{C}_{2} \\
\text { radical }^{6}\end{array}$ \\
\hline
\end{tabular}


TABLE 7.3 (continued)

\begin{tabular}{|c|c|c|c|}
\hline $\mathrm{CF}_{3} \mathrm{CN}$ & $\mathrm{CF}_{3}+\mathrm{CN}$ & 135 & \\
\hline $\mathrm{CH}_{2} \mathrm{DCH}_{2} \mathrm{Cl}$ & {$\left[\begin{array}{l}\mathrm{C}_{2} \mathrm{H}_{3} \mathrm{D}+\mathrm{HCl} \\
\rightarrow \mathrm{C}_{2} \mathrm{H}_{4}+\mathrm{DCl}\end{array}\right.$} & 172 & $\begin{array}{r}\mathrm{C}_{2} \mathrm{H}_{3} \mathrm{D} \stackrel{n \hbar \Omega}{\longrightarrow} \mathrm{C}_{2} \mathrm{H}_{2}+\mathrm{HD}^{172} \\
\mathrm{C}_{2} \mathrm{HD}+\mathrm{H}_{2} \\
\mathrm{C}_{2} \mathrm{H}_{4} \stackrel{n \hbar \Omega}{\longrightarrow} \mathrm{C}_{2} \mathrm{H}_{2}+\mathrm{H}_{2}{ }^{172}\end{array}$ \\
\hline $\mathrm{CH}_{2} \mathrm{FCH}_{2} \mathrm{Cl}$ & $\stackrel{\mathrm{C}_{2} \mathrm{H}_{3} \mathrm{~F}+\mathrm{HCl}}{\mathrm{C}_{2} \mathrm{H}_{3} \mathrm{Cl}+\mathrm{HF}}$ & 37 & $\mathrm{C}_{2} \mathrm{H}_{3} \mathrm{Cl} \stackrel{n \hbar \Omega}{\longrightarrow} \mathrm{C}_{2} \mathrm{H}_{2}+\mathrm{HCl}^{37}$ \\
\hline $\mathrm{CH}_{2} \mathrm{FCH}_{3}$ & $\mathrm{C}_{2} \mathrm{H}_{4}+\mathrm{HF}$ & 56 & \\
\hline $\mathrm{CHF}_{2} \mathrm{CH}_{3}$ & $\mathrm{C}_{2} \mathrm{H}_{3} \mathrm{~F}+\mathrm{HF}$ & 56 & \\
\hline $\mathrm{CH}_{3} \mathrm{CF}_{2} \mathrm{Cl}$ & $\mathrm{C}_{2} \mathrm{H}_{2} \mathrm{~F}_{2}+\mathrm{HCl}$ & 49 & \\
\hline $\mathrm{CH}_{3} \mathrm{CCl}_{3}$ & $\mathrm{C}_{2} \mathrm{H}_{2} \mathrm{Cl}_{2}+\mathrm{HCl}$ & 49 & \\
\hline $\mathrm{CF}_{3} \mathrm{CF}_{2} \mathrm{Cl}$ & {$\left[\begin{array}{l}\mathrm{C}_{2} \mathrm{~F}_{5}+\mathrm{Cl} \\
\rightarrow \mathrm{CF}_{3}+\mathrm{CF}_{2} \mathrm{Cl}\end{array}\right.$} & 49 & \\
\hline $\mathrm{C}_{3} \mathrm{H}_{7} \mathrm{Br}$ & $\mathrm{C}_{3} \mathrm{H}_{6}+\mathrm{HBr}$ & 163 & \\
\hline $\mathrm{CH}_{3} \mathrm{OH}$ & $\stackrel{\longrightarrow \mathrm{CH}_{3}+\mathrm{OH}}{\longrightarrow \mathrm{CH}_{2} \mathrm{OH}+\mathrm{H}(?)}$ & $\begin{array}{l}100 \\
85\end{array}$ & $\begin{array}{l}\text { Visible luminescence of } \mathrm{OH} \\
\text { and } \mathrm{CH} \text { radicals }\end{array}$ \\
\hline $\mathrm{CH}_{3} \mathrm{CN}$ & $\mathrm{CH}_{3}+\mathrm{CN}$ & 25,101 & $\begin{array}{l}\text { Visible luminescence of } \mathrm{CH} \\
\text { and } \mathrm{C}_{2} \text { radicals }^{6}\end{array}$ \\
\hline
\end{tabular}

two molecules the dissociation by this channel is preferential. The most probable cause of this effect consists in the presence of a potential barrier for the "advantageous" channel. Indeed, in all these cases the "advantageous" channel is associated with elimination of molecules and rearrangement of molecular bonds. It is well known (see Section 2.4) that for such a process there may be a barrier the height of which $E_{A}$ for the direct reaction to a greater or lesser degree exceeds the value of $H$ for this process. Also, $E_{A}$ may exceed the dissociation energy of the next channel and so the dissociation here may be more effective than by the first channel. We shall return to this point in the next section where we consider experimental proof of the presence of a barrier for $\mathrm{CF}_{2} \mathrm{Cl}_{2}$ based on measuring the vibrational energy in the $\mathrm{CF}_{2}$ radical. $^{41}$

Much research has been directed on the MP dissociation products of the $\mathrm{SF}_{6}$ molecule. Energetically the most advantageous dissociation channel is the reaction

$$
\mathrm{SF}_{6} \rightarrow \mathrm{SF}_{5}+\mathrm{F}
$$


Nevertheless, on the basis of an analysis of the final dissociation products ${ }^{44,45}$ it was proposed the possibility of dissociation by the channel $\mathrm{SF}_{6} \rightarrow \mathrm{SF}_{4}+\mathrm{F}_{2}$ even though energetically it was less advantageous. Later ${ }^{15-16,46-47}$ different methods of diagnostics (see Section 7.1.1) were used to show conclusively that, in $\mathrm{MP}$ dissociation of $\mathrm{SF}_{6}$, $F$ atoms rather than $F_{2}$ molecules are formed.

Direct diagnostics of the radicals formed by MP dissociation of $\mathrm{SF}_{6}$ and other molecules became possible when the molecular beam technique was introduced into these experiments. The radicals acquiring a transverse momentum component in the process of dissociation were studied $^{4,40,48-49,5}$ by the research group at the University of California, Berkeley, USA. Particular attention was focused on diagnostics of the MP dissociation of the $\mathrm{SF}_{6}$ molecule. The measurements were taken using the mass peaks of the $\mathrm{SF}_{3}^{+}$and $\mathrm{SF}_{2}^{+}$ions formed in the ionization chamber (see Figure 7.1). The theoretical ratios of these peaks in the ionization of $\mathrm{SF}_{6}, \mathrm{SF}_{5}$ and $\mathrm{SF}_{4}$ are approximately equal to 6,4 and 2 respectively. Figure 7.9 shows angular distributions of the ions in

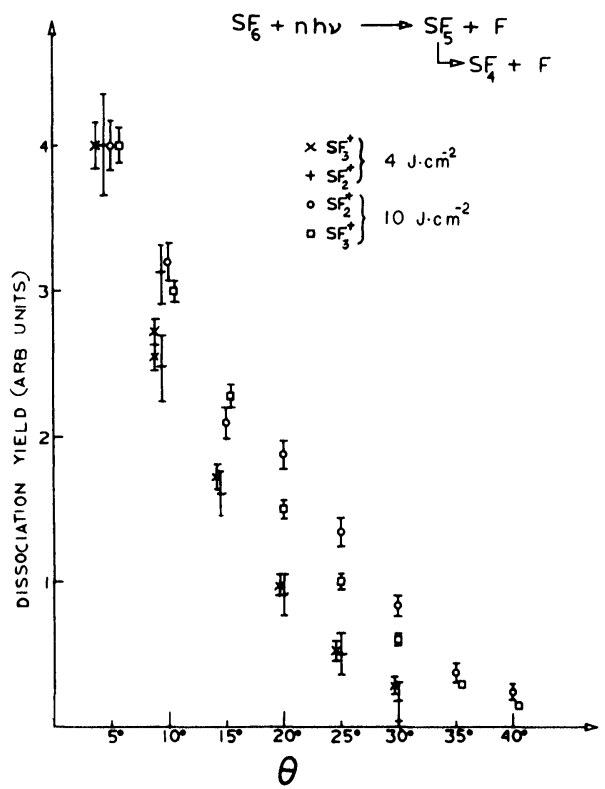

FIGURE 7.9 MP dissociation of $\mathrm{SF}_{6}$ molecules in a molecular beam. ${ }^{48}$ The angular distributions of the $\mathrm{SF}_{3}^{+}$and $\mathrm{SF}_{4}^{+}$ions are given for two values of laser pulse fluence. 
arbitrary units. At a relatively small excess of laser pulse energy fluence $\left(\Phi=4 \mathrm{~J} / \mathrm{cm}^{2}\right)$ over its threshold value the ratio observed for all angles was $\left[\mathrm{SF}_{3}^{+}\right] /\left[\mathrm{SF}_{2}^{+}\right] \simeq 4$ within the limits of experimental error, which corresponded to the $\mathrm{SF}_{5}$ radical. When the fluence is increased $(\Phi=$ $10 \mathrm{~J} / \mathrm{cm}^{2}$ ) the ratio $\left[\mathrm{SF}_{3}^{+}\right] /\left[\mathrm{SF}_{2}^{+}\right]$in the region of large angles decreases considerably. The authors of the experiments believe that this fact is explained by the second stage of MP dissociation as a result of which the $\mathrm{SP}_{5}$ radicals formed by reaction (7.4) further dissociate under the action of the radiation.

The case of $\mathrm{SF}_{6}$ is quite typical. The process in which successive breaking of two and even more bonds takes place can be observed rather frequently. The polyatomic products formed at the first stage of MP dissociation can go on absorbing the radiation particularly when they are vibrationally excited. Such successive dissociation can last as long as the laser pulse, and so long as the fragments can absorb energy. Successive dissociation of a molecule and the resulting fragments is responsible for the birth of such diatomic radicals as $\mathrm{C}_{2}, \mathrm{CH}$, and $\mathrm{CN}$ in the MP dissociation of some hydrocarbons. For illustration, we may present the sequence of the basic processes resulting in simultaneous formation of the $\mathrm{C}_{2}$ and $\mathrm{CN}$ radicals in MP dissociation of $\mathrm{C}_{2} \mathrm{H}_{3} \mathrm{CN}^{51}$

$$
\begin{array}{ll}
\mathrm{C}_{2} \mathrm{H}_{3} \mathrm{CN} \rightarrow \mathrm{H}_{2}+\mathrm{C}_{2} \mathrm{HCN} & \left(\Delta H^{\circ}=14716 \mathrm{~cm}^{-1}\right) \\
\mathrm{C}_{2} \mathrm{H}_{3} \mathrm{CN} \rightarrow \mathrm{H}+\mathrm{C}_{2} \mathrm{H}_{2} \mathrm{CN} & \left(\Delta H^{\circ}=38043 \mathrm{~cm}^{-1}\right) \\
\mathrm{C}_{2} \mathrm{H}_{2} \mathrm{CN} \rightarrow \mathrm{H}+\mathrm{C}_{2} \mathrm{HCN} & \left(\Delta H^{\circ}=13879 \mathrm{~cm}^{-1}\right)
\end{array}
$$

The radical $\mathrm{C}_{2} \mathrm{HCN}$ formed by $(7.5)$ and (or) $(7.6,7.7)$ is subjected to further dissociation

$$
\begin{aligned}
\mathrm{C}_{2} \mathrm{HCN} & \rightarrow \mathrm{H}+\mathrm{C}_{2} \mathrm{CN} \quad\left(\Delta H^{\circ}=46070 \mathrm{~cm}^{-1}\right) \\
\mathrm{C}_{2} \mathrm{CN} & \rightarrow \mathrm{C}_{2}+\mathrm{CN}
\end{aligned}
$$

The authors ${ }^{51}$ note that, despite a great difference in the values of $\Delta H^{\circ}$ in reactions (7.5) and (7.6), the latter can occur since reaction (7.5) has an activation barrier $E_{A} \simeq 28000 \mathrm{~cm}^{-1}$.

It is also of interest to clarify the temporal behayior of the reaction radicals in time. We may point out conventionally two processes. The first of them is connected with MP dissociation during a laser pulse, and the main contribution to this process is given by those molecular 
states excited over the dissociation limit the decay rate of which $k\left(E_{\mathrm{vib}}\right)$ exceeds the reverse duration of laser pulse $k\left(E_{\mathrm{vib}}\right)$. The research using the LIF method that provides rather high time resolution shows that in some cases this process is dominant (see Figure 7.10). The second process is connected with the dissociation of the molecules excited by a laser pulse to the states which lie comparatively not high above the dissociation limit so that their dissociation rate is less than $\tau_{p}^{-1}$. Thus, the contribution of these two processes depends on the level of molecular excitation over the dissociation limit $E_{\mathrm{vib}}-D$ and the relation between $\tau_{p}$ and $k\left(E_{\mathrm{vib}}\right)$. The possibility of experimental separation of these two processes was demonstrated ${ }^{52}$ where the MP dissociation of the $\mathrm{C}_{2} \mathrm{H}_{4} \mathrm{~F}_{2}$ molecule was studied by recording with time resolution the IR fluorescence of the resultant HF molecules. As a result, estimates were obtained for the decay times of the levels contributing to dissociation during a laser pulse and after its action, along with the relative contributions of the two processes. This experiment in its essense was similar to experiments on the direct study of the energy distribution of the fragments. Such experiments are discussed in the next section. It should be noted that recording of MP dissociation products of molecular ions with time has been achieved..$^{53,54}$

The cases considered show to what extent the effects arising in molecular dissociation changes as a result of their MP excitation with varied IR radiation. Nevertheless, with all this variety all the results obtained so far are consistent with the statistical model the main assumption of which is fast redistribution of vibrational energy over all molecular modes. There have been so far no reliable experiments which would be indicative of an observation of mode-selective dissoci-

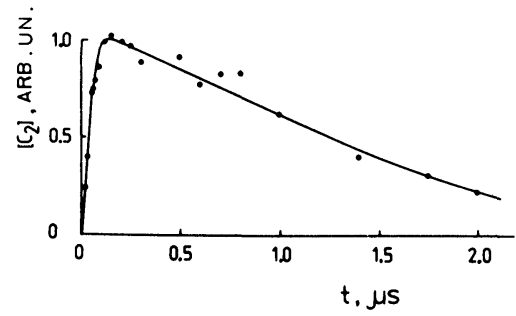

FIGURE 7.10 MP dissociation of $\mathrm{C}_{2} \mathrm{H}_{4}$ molecules. ${ }^{129}$ The dependence of the concentration of $\mathrm{C}_{2}$ radicals formed in the process of MP dissociation on time. (The decrease of the signal is connected with the radical flight from the zone of probing.) 
ation in MP excitation of molecules. $\dagger$ And conversely, the available results are in agreement with the hypothesis on stochasticity of vibrational motion for highly excited molecules.

\subsubsection{Energy distribution of MP dissociation products}

Molecules excited as they absorb IR radiation dissociate if their energy $E_{\text {vib }}$ exceeds the dissociation energy $D$. In this case the energy excess $\Delta E=E_{\text {vib }}-D$ is distributed between different degrees of freedom of the resulting fragments. Before considering the particular distributions of this energy we are going to review briefly what is predicted by the RRKM theory on the value of the energy $\Delta E$. The estimations done in Chapter 4 (see Section 4.4.2) show that for typical experimental conditions with the laser pulse duration $\tau_{p} \simeq 10^{-7} \mathrm{~s}$ the real excitation over the dissociation limit doesn't exceed $\Delta E \leqslant(0.1-0.3) \mathrm{D}$ when the number of atoms in the molecule is $\leqslant 7$.

The results of experiments with molecular beams agree with these estimations. In the $\mathrm{SF}_{6}$ molecule, ${ }^{48}$ for example, it was concluded that the value of excess over the dissociation limit, with the $\mathrm{CO}_{2}$ laser radiation fluence $\Phi=(4-10) \mathrm{J} / \mathrm{cm}^{2}$, ranged from 6 to 10 quanta. For molecules with a smaller number of atoms the overexcitation is lower which also agrees with the conclusions of the RRKM theory. For the $\mathrm{CF}_{3} \mathrm{Br}$ molecule at the same values of $\Phi$ the maximum excess over the dissociation limit comes to three quanta.

Now consider how the energy excess $\Delta E$ is distributed over the degrees of freedom of fragments. Let us begin with the translational degrees.

Translational energy distribution. According to the RRKM theory, the probability of conversion of the excessive molecular energy $E_{\mathrm{vib}}-$ $D$ equal to $E_{\text {TRANS }}$ will be transformed into translational energy is described by the distribution function

$$
g\left(E_{\text {TRANS }}\right)=\text { const. } \frac{d}{d E_{\text {TRANS }}} R\left(E_{\mathrm{vib}}-\mathrm{D}-\mathrm{E}_{\text {TRANS }}\right)
$$

where $R$ is the number of activated complex states in the energy range between $D$ and $E_{\mathrm{vib}}-E_{\text {TRANS. }}$. Since the density of activated complex

† We are not considering here a special case of "site-selective" excitation of large molecules (see Section 6.1.2). 
states $d R / d E$ grows quickly with increasing energy the maximum of the distribution function $g\left(E_{\text {TRANS }}\right)$ corresponds to the zero value of recoil energy.

The conclusions of the RRKM theory are in good agreement with the results of experiments in molecular beams when the molecular dissociation is a simple two-center reaction. The measured angular and velocity distributions of dissociation fragments of $\mathrm{SF}_{6}, \mathrm{C}_{2} \mathrm{~F}_{5} \mathrm{Cl}$, $\mathrm{N}_{2} \mathrm{~F}_{4}$ and a number of halogenated methanes ${ }^{40,48}$ are consistent with the calculated functions. This enables us to estimate the level of molecular excitation over the dissociation limit. The characteristic feature of these distributions is the presence of a maximum at zero recoil energy. (For angular distribution this corresponds to the zero value of angle $\theta$ (see Figure 7.9).) At two-center bond breaking only a small part of excess energy $\Delta E$ (less than $20-30 \%$ ) is converted to the translational energy of fragments. In MP dissociation of $\mathrm{SF}_{6}$ (reaction (7.4)) of the total overexcitation energy $\Delta E \simeq 9000 \mathrm{~cm}^{-1}$ only about $1000 \mathrm{~cm}^{-1}$ is converted to translational energy. The rest of the energy remains in the vibrational and rotational degrees of freedom of the $\mathrm{SF}_{5}$ radical.

The presence of a barrier along the reaction coordinate as often exists for three- or four-center molecular decay, changes the behavior of the recoil energy distribution. The maximum of this distribution can be observed, for example, at some non-zero finite value of energy. The experiments with $\mathrm{CHF}_{2} \mathrm{Cl}, \mathrm{CHFCl}_{2}, \mathrm{CH}_{3} \mathrm{CCl}_{3}, \mathrm{CH}_{3} \mathrm{CF}_{2} \mathrm{Cl}^{50}$ where elimination of the $\mathrm{HCl}$ molecule was observed show that, besides the possible transfer of a part of excess energy $\Delta E, \dagger$ a considerable part (up to $20 \%$ ) of potential energy of reverse reaction barrier is directly transferred to translational degrees of freedom. In the four-center dissociation of the $\mathrm{CH}_{3} \mathrm{CCl}_{3}$ molecule ${ }^{50}$

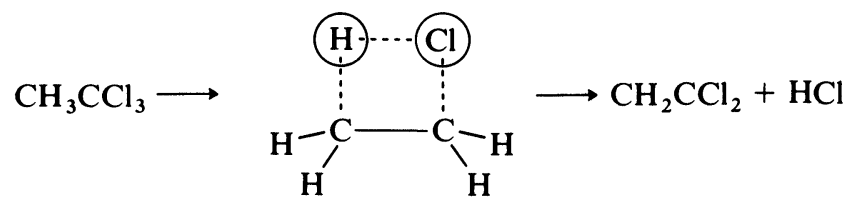

the reverse reaction barrier equals $14680 \mathrm{~cm}^{-1}$ and thus the fragments acquire considerable translational energy $\bar{E}_{\text {TRANS }} \simeq 2800 \mathrm{~cm}^{-1}$.

$\dagger$ We mean here an excess over the direct reaction barrier. 
The formation of a barrier is a result of the interaction of the fragments even after they have formed critical reactive configurations. Therefore it is impossible to predict correctly how the energy is distributed over all degrees of freedom of fragments without additional assumptions on the form of the potential surfaces along the reaction coordinate. The value of $E_{\text {TRANS }}$ calculated with the use of the RRKM theory ${ }^{50}$ within the frameworks of statistical distribution over degrees of freedom may differ considerably from the experimental value; in the case of $\mathrm{CH}_{3} \mathrm{CCl}_{3}$ it is smaller by four times.

Distribution over vibrational and rotational degrees of freedom. The basic results have been obtained using the laser induced fluorescence method. The possibility of its application in measuring vibrational and rotational distribution is obvious enough and consists in probing different vibrational-rotational states in the electronic absorption spectra of dissociation products with a tunable laser. The typical results of this kind of experiment for $\mathrm{C}_{2}$ radicals is shown in Figure 7.11. Knowing the values of Frank-Condon factors, it is possible to determine the population ratio for any pair of vibrational-rotational levels from the ratio of fluorescence signals and, as a result, to obtain information on the distribution function.

The distribution of vibrational energy in the $\mathrm{CF}_{2}$ radical formed by MP dissociation of $\mathrm{CF}_{2} \mathrm{Cl}_{2}, \mathrm{CF}_{2} \mathrm{Br}_{2}$ and $\mathrm{CF}_{2} \mathrm{HCl}$ was studied ${ }^{41,55}$ and it was shown that the distribution of vibrational energy in the $\mathrm{CF}_{2}$ radical for these three molecules is Boltzmann-like. For illustration, we will consider the $\mathrm{CF}_{2} \mathrm{Cl}_{2}$ molecule. In Section 7.2.1 it has been

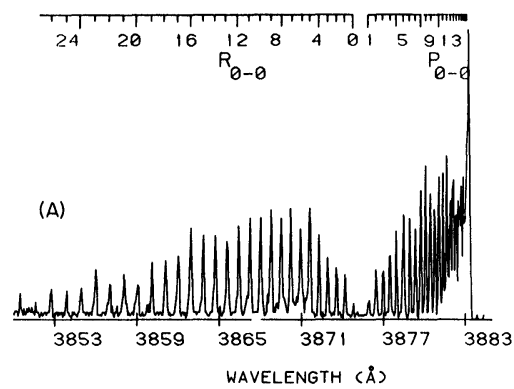

FIGURE 7.11 Spectrum of laser-induced fluorescence of the $C_{2}$ radical during MP dissociation of $\mathrm{C}_{2} \mathrm{H}_{3} \mathrm{CN}$. The signal is observed at the transition $\mathrm{B} \rightarrow \mathrm{X}(0,1)$ with the dye laser radiation scanned along the band $\mathrm{X} \rightarrow \mathrm{B}(0,0)$. 
already noted that the preferential role of the channel of elimination of the $\mathrm{Cl}$ atom, as compared to that of the $\mathrm{Cl}_{2}$ molecule, is apparently caused by the presence of a barrier for the second reaction. In this case one would expect that the dissociation of $\mathrm{CF}_{2} \mathrm{Cl}_{2}$ into the $\mathrm{CF}_{2}$ radical and the $\mathrm{Cl}_{2}$ molecule mainly occurs near the barrier top. The measurements of the vibrational distribution in the $\mathrm{CF}_{2}$ radical ${ }^{41}$ fully support these conclusions. It was found that as the laser pulse energy fluence varied from 1.5 to $6 \mathrm{~J} / \mathrm{cm}^{2}$ the vibrational temperature $(T=$ $1050 \pm 100 \mathrm{~K}$ ) remained almost constant even though the radical yield changed by about an order. Based on these results ${ }^{41}$ it was concluded that molecular dissociation actually comes about mainly near the top of the barrier the height of which is estimated by them to be $6900 \mathrm{~cm}^{-1}$.

Measurements have been taken of the rotational energy distribution in the radicals formed by MP dissociation with no variations from the Boltzmann rotational distribution observed. The results are presented in Table 7.4, where the rotational temperatures of the radicals investigated are given as well as their vibrational temperatures for the cases when they were measured in parallel. It may be seen that in most cases the vibrational and rotational temperatures differ greatly. For the $\mathrm{CF}_{2}$ radical formed by $\mathrm{MP}$ dissociation of $\mathrm{CF}_{2} \mathrm{CClF}$ the temperatures differ even in different vibrational modes. The experiments with $\mathrm{CF}_{2} \mathrm{CClF}$ enable us to conclude that the rotational distribution as well as the average translational energy $\dagger$ of the dissociation products of this molecule do not correlate with their vibrational energy.

The results presented in Table 7.4 demonstrate vividly the potential of the fluorescence method. The results, particularly the Boltzmann type distribution as well as the difference between vibrational and rotational temperatures, have not however been interpreted yet.

Interesting results were obtained ${ }^{56}$ when the energy distribution of the vibrationally excited $\mathrm{HF}$ molecules formed as a result of their elimination from $\mathrm{CH}_{2} \mathrm{CHF}, \mathrm{CH}_{3} \mathrm{CH}_{2} \mathrm{~F}, \mathrm{CH}_{3} \mathrm{CHF}_{2}, \mathrm{CH}_{2} \mathrm{CF}_{2}$ was studied observing their IR fluorescence spectra. For these molecules, too, the rotational distribution of fragments was Boltzmann-like. Study

$\dagger$ The translational energy of the fragments may be determined from the measurements of their velocities at which they leave the region of probing (see e.g., Figure 7.10 and the caption to it). It is important that this method gives an opportunity to determine the translational energies for particular quantum states of the fragments. Some results of this kind of experiments are presented in Table 7.4. 
TABLE 7.4

Energy distribution of MP dissociation fragments

\begin{tabular}{|c|c|c|c|c|c|c|}
\hline Molecule & Fragment & $\begin{array}{c}\text { Laser } \\
\text { pulse } \\
\text { energy } \\
\text { fluence, } \\
\mathrm{J} / \mathrm{cm}^{2}\end{array}$ & $\begin{array}{c}\text { Vibrational } \\
\text { temperature, } \\
\text { K }\end{array}$ & $\begin{array}{c}\text { Rotational } \\
\text { temperature, } \\
\text { K }\end{array}$ & $\begin{array}{c}\text { Average } \\
\text { translational } \\
\text { energy, }{ }^{\mathrm{a}} \\
\mathrm{kcal} / \mathrm{mol}\end{array}$ & Refs. \\
\hline $\begin{array}{l}\mathrm{CF}_{2} \mathrm{Cl}_{2} \\
\mathrm{CF}_{2} \mathrm{Br}{ }_{2} \\
\mathrm{CF}_{2} \mathrm{HCl}\end{array}$ & $\begin{array}{l}\mathrm{CF}_{2} \\
\mathrm{CF}_{2} \\
\mathrm{CF}_{2}\end{array}$ & $\begin{array}{l}1.5-6 \\
1.5-6 \\
2.4-26\end{array}$ & $\begin{array}{c}1050 \pm 100 \\
790 \pm 70 \\
1160 \pm 100\end{array}$ & $\begin{array}{r}550 \\
450 \\
2000\end{array}$ & & $\begin{array}{l}41,55 \\
55 \\
55\end{array}$ \\
\hline $\mathrm{CH}_{3} \mathrm{OH}$ & $\left\{\begin{array}{l}\mathrm{OH} \\
\mathrm{CH}\end{array}\right.$ & $\begin{array}{l}150 \\
150\end{array}$ & & $\begin{array}{l}1250 \pm 400 \\
1200 \pm 400\end{array}$ & 100 & 100 \\
\hline $\begin{array}{l}\mathrm{CH}_{3} \mathrm{CN} \\
\mathrm{C}_{2} \mathrm{H}_{3} \mathrm{CN}\end{array}$ & $\left\{\begin{array}{l}\mathrm{CN} \\
\mathrm{C}_{2} \\
\mathrm{CN}\end{array}\right.$ & $\begin{array}{c}300 \\
10-80 \\
10-80\end{array}$ & 425 & $\begin{array}{c}664 \\
700 \pm 200 \\
1000 \pm 200\end{array}$ & $\begin{array}{c}101 \\
1.1 \pm 0.3\end{array}$ & $\begin{array}{c}51,102 \\
51\end{array}$ \\
\hline $\mathrm{CF}_{2} \mathrm{CFCl}$ & $\left\{\begin{array}{l}\mathrm{CFCl} \\
\mathrm{CF}_{2}\end{array}\right.$ & $\begin{array}{l}50 \\
50\end{array}$ & $\left\{\begin{array}{c}1550 \pm 300\left(\nu_{2}\right) \\
1860 \pm 250\left(\nu_{2}\right) \\
1100\left(\nu_{1}, \nu_{3}\right)\end{array}\right.$ & 400 & $\begin{array}{l}0.3 \\
0.4\end{array}$ & $\begin{array}{l}42 \\
42\end{array}$ \\
\hline
\end{tabular}

${ }^{a}$ Measured in center-of-mass system.

of the vibrational distribution, however, shows that it cannot be adequately described only by the temperature of the corresponding Boltzmann distribution. The results of the measurements show that from $16 \%$ to $31 \%$ (depending on the molecule) of potential energy of the reverse reaction barrier is directly spent on vibrational excitation of HF. This is about one order higher than the result calculated by the RRKM theory on the assumption of statistical energy distribution.

These results as well as the results of translational energy distribution considered before show once again that in the presence of a potential barrier we cannot calculate correctly the energy distribution of the fragments without knowing the potential surface along the reaction coordinate. At the same time the available data shows that the character of the energy distribution over different degrees of freedom of the fragments apparently depends only slightly on laser IR excitation parameters and is governed predominantly by the level of molecular excitation. This makes possible wide application of IR multiphoton molecular excitation in combination with proper diagnostic methods of radical determination in studying the process of unimolecular dissociation of highly excited molecules and the potential surface structure of these molecules. 


\subsubsection{Visible luminescence in the case of MP IR excitation of molecules}

We considered above the characteristics of the dissociation of molecules resulting from their MP excitation followed by the formation of fragments in the ground electronic state. But in MP excitation of some molecules it was possible to observe visible luminescence which was associated with the luminescence of electronically excited radicals (see column 4 in Table 7.3). As a matter of fact, the effect of collisionless molecular dissociation by IR radiation was disclosed by detecting visible luminescence in experiments with $\mathrm{SiF}_{4}{ }^{1}$ and $\mathrm{BCl}_{3}{ }^{2}$ Nevertheless, the mechanism of the formation of electronically excited fragments has remained until recently probably the least understood point in the entire problem of MP excitation and dissociation of molecules.

Recent studies have clarified this point to some extent. The origination of the visible luminescence is apparently caused by a nonadiabatic coupling of the ground electronic state with the excited electronic term when the energy of the latter is lower than the dissociation limit from the ground term (see the end of Section 2.7). This effect is often called inverse electronic relaxation (IER). ${ }^{57}$ It results from a breakdown of the Born-Oppenheimer approximation within which the vibrational states for different electronic terms are not interacting. Interaction, however, causes the real eigenvalues of the molecules to be an isoenergetic state superposition of the ground and excited electronic terms. In multiple photon IR excitation of such states their one-photon decay to the ground state becomes possible, with a quantum of visible radiation emitted.

One of the main predictions of the IER theory ${ }^{57}$ is a considerable increase in the luminescence lifetime $\tau_{0}^{l}$ as compared to the typical time of dipole-allowed transition $\tau_{1}^{a d}$ in an adiabatic approximation. This increase depends (see Section 2.128) on the ratio of vibrational state densities in the ground and excited electronic states and may be as high as several orders. So, long luminescence, in a range from several tens of microsecond to milliseconds (for $\mathrm{OsO}_{4} \tau_{0}^{l} \simeq$ $1.5 \cdot 10^{-3} \mathrm{~s}^{58}$ ), is a characteristic feature which in many cases has made it possible to ascertain the relation of this luminescence to the IER effect.

Long-lived luminescence was observed in IR multiple photon excitation of tetramethyldioxetane ${ }^{.9} \quad \mathrm{~S}_{2} \mathrm{C}_{2} \mathrm{~F}_{4} ;^{60} \quad \mathrm{C}_{2} \mathrm{H}_{4}, \mathrm{C}_{3} \mathrm{H}_{8}, \mathrm{C}_{2} \mathrm{H}_{3} \mathrm{Cl}$, $\mathrm{C}_{2} \mathrm{H}_{3} \mathrm{CHO} ;{ }^{61-62} \mathrm{OsO}_{4} ;{ }^{7} \mathrm{CrO}_{2} \mathrm{Cl}_{2}{ }^{9}$ 
Rather well studied is visible luminescence arising in MP excitation of $\mathrm{OsO}_{4}$. It follows from the results ${ }^{7,58,63}$ that the most probable mechanism of luminescence is as follows

$$
\begin{aligned}
& \mathrm{OsO}_{4}+n \hbar \Omega \rightarrow \mathrm{OsO}_{4}{ }^{*} \mathrm{OsO}_{3}+\mathrm{O} \\
& \mathrm{OsO}_{3}+m \hbar \Omega \mathrm{OsO}_{4}+\hbar \Omega_{\text {vis }}^{(1)} \\
& \mathrm{OsO}_{3}{ }^{*} \mathrm{OsO}_{2}+\mathrm{O}(?) \\
& \mathrm{OsO}_{3}+\hbar \Omega_{\text {vis }}^{(2)}
\end{aligned}
$$

The highly vibrationally excited $\mathrm{OsO}_{4}^{*}$ molecules formed by MP excitation can emit a visible radiation quantum or dissociate to form $\mathrm{OsO}_{3}$ (7.12). The latter, when absorbing then the IR radiation, can be highly excited and emit another visible radiation quantum as a result of one more process of IER (7.13).

Processes (7.12) and (7.13) in $\mathrm{OsO}_{4}$ are separated on the laser radiation energy fluence scale (Figure 7.12). With $\Phi<3 \mathrm{~J} / \mathrm{cm}^{2}$ the main contribution to the luminescence signal is made by the luminescence of the $\mathrm{OsO}_{4}$ molecule itself (reaction (7.12)). With $\Phi>3 \mathrm{~J} / \mathrm{cm}^{2}$ luminescence "quenching" arises which is caused by decreasing concentration of $\mathrm{OsO}_{4}^{*}$ because of their dissociation. With $\Phi>4 \mathrm{~J} / \mathrm{cm}^{2}$ a new type of luminescence arises which is apparently connected with luminescence of the $\mathrm{OsO}_{3}^{*}$ radical according to reaction (7.13). Further increase in laser radiation energy causes the luminescence signal, with $\Phi>$ $10 \mathrm{~J} / \mathrm{cm}^{2}$, to decrease again, because of dissociation of the $\mathrm{OsO}_{3}$ radical this time.

The dependence of the luminescence signal on $\Phi$ in MP excitation of $\mathrm{CrO}_{2} \mathrm{Cl}_{2}$ (curve 2, Figure 7.12) is a little different in character but from the results ${ }^{63-65}$ it follows that in this case, too, the luminescence source is the $\mathrm{CrO}_{2} \mathrm{Cl}_{2}$ molecule itself, as well as $\mathrm{CrO}_{2} \mathrm{Cl}$ radicals, and then probably CrO.

The fact that visible luminescence is associated with the intermediate products of MP dissociation has been established in time-of-flight experiments ${ }^{62}$ for $\mathrm{C}_{2} \mathrm{H}_{4}\left(\tau_{0}^{l} \simeq 8 \mu \mathrm{s}\right), \mathrm{C}_{2} \mathrm{HCl}_{3}\left(\tau_{0}^{l} \simeq 40 \mu \mathrm{s}\right), \mathrm{C}_{2} \mathrm{H}_{3} \mathrm{CN}\left(\tau_{0}^{l} \simeq\right.$ $22 \mu \mathrm{s})$. In the last case the luminescence source is the $\mathrm{C}_{2} \mathrm{HCN}$ or $\mathrm{C}_{2} \mathrm{CN}$ radicals formed by reactions (7.6), (7.7) or (7.8). 


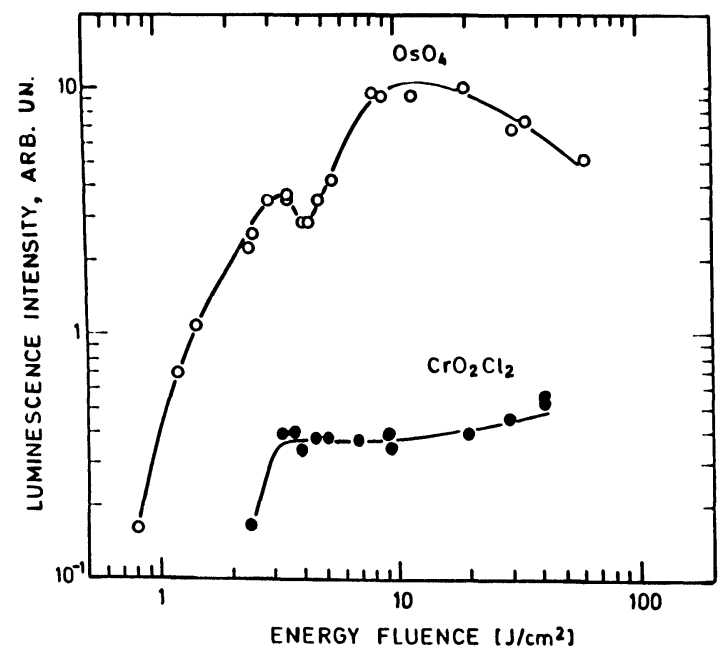

FIGURE 7.12 Dependence of the luminescence signal intensity on the $\mathrm{CO}_{2}$ pulse fluence in the process of MP dissociation of $\mathrm{OsO}_{4}$ and $\mathrm{CrO}_{2} \mathrm{Cl}_{2}{ }^{63}$

In conclusion of this short section we must note that studies into visible luminescence in MP excitation are actually far from complete. It is only recently that inverse electron relaxation has been accepted. This effect is the most probable physical mechanism of the formation of electronically excited fragments. This conclusion can be apparently applied to the results of early experiments. The IER effect still requires further research. Specifically, the measured values of luminescence life time $\tau_{0}^{l}$ are only in qualitative agreement with those predicted theoretically. The difference may be rather significant. For example, the measured luminescence life time of the $\mathrm{CrO}_{2} \mathrm{Cl}_{2}$ molecule $\tau_{0}^{l} \simeq$ $160 \mu \mathrm{s}^{65}$ which is much larger than $\tau_{1}^{a d} \sim 1 \mu \mathrm{s}$ but almost two orders less than the calculated value. The role of different channels of quenching, including that from IR fluorescence, remains to be seen. Future studies will probably enable us to understand better this interesting effect.

\subsection{Isomerization of multiple photon excited molecules}

Besides the processes related to fragmentation of polyatomic molecules, there is one more unimolecular photoprocess, that is, the 
isomerization of molecules following their excitation. Photoisomerization of electronically excited molecules is a well known effect. ${ }^{66}$ This process is of great importance in photobiology, for example in the molecular mechanism of vision (photoisomerization of rhodapsin molecules). One may expect that strong vibrational excitation of a polyatomic molecule by an intense IR field can also cause rearrangement of atoms and (or) bonds stably. It is interesting, in principle, to observe such a process in a strong IR field since it allows another probe of the effect of multiphoton absorption of IR radiation by molecules when the excited levels are lower than the level essential for molecular dissociation. It may also be important to achieve the selective isomerization of molecules in a strong IR field for widening the scope of isotope separation by IR laser radiation for the following reasons. First, isomerization gives a stable molecule with no need for chemical scavenging of dissociation fragments. It is also beneficial for the conservation of selectivity from secondary photochemical processes. Second, the isomerization energy may be much lower than the dissociation energy which allows the isomerization process to be performed with lower consumption of energy. On the other hand, because of a comparatively low energy barrier the process of isomerization is more sensitive to thermal heating than the process of dissociation.

There is one more factor which distinguishes dissociation from isomerization. In the first case the energy stored by the molecule is spent to break one of the molecular bonds, and this process is rather fast. In the second case the molecule with its energy being higher than the potential barrier (Figure 7.13) is able to change its structure. But, unlike the process of dissociation, the molecule can for a long time oscillate between two isomeric forms in the ground electronic state. To stabilize the molecule in one of the forms we should take away a part of the energy. Since the rate of radiative transition in the IR range is small $\left(\tau=10^{-3}-10^{-2} \mathrm{~s}\right)$ stabilization of the molecule in most cases results from vibrational deexcitation during collisions. So the effect of collisions on the characteristics of isomerization in MP excitation is expected to be stronger than in case of dissociation.

Particular emphasis has been given so far to molecular dissociation, whereas there is a much smaller body of work concerning isomerization. In the first work ${ }^{67}$ consideration was given to isomerization of trans-2-butene by $\mathrm{CO}_{2}$ laser pulses. In addition to dissociation of 


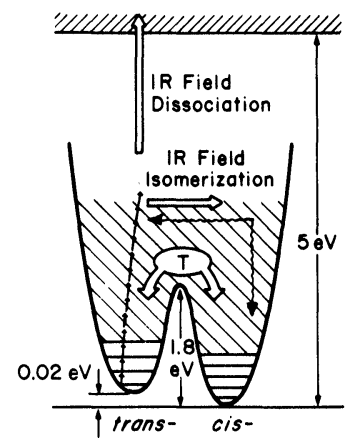

FIGURE 7.13 Dissociation and isomerization resulting from MP excitation of molecules. Example of $\mathrm{C}_{2} \mathrm{H}_{2} \mathrm{Cl}_{2}$.

trans-2-butene, the formation of cis-2-butene was observed in the same work, that is, the processes of dissociation and isomerization occurred approximately with the same rate.

The effect of trans-cis-isomerization in MP excitation of $\mathrm{C}_{2} \mathrm{H}_{2} \mathrm{Cl}_{2}$ molecules has been studied ${ }^{68}$ in detail. Particular emphasis was placed upon a search for experimental conditions under which the threshold for isomerization would be much lower than for dissociation. In the molecule concerned, however, this aim was not attained, and after comprehensive studies the experimental conditions were found under which the isomerization rate was several times higher than the dissociation rate. It was also proved that the isomerization of the $\mathrm{C}_{2} \mathrm{H}_{2} \mathrm{Cl}_{2}$ molecule was nonthermal. Some molecules have been found that under intense IR radiation only isomeric transformations occur without the formation of any other products. They are $\mathrm{CH}_{3} \mathrm{NC},{ }^{69}$ and deuterated 1,5 -hexadien $\mathrm{e}^{70}$ for which the possibility of isotope-selective isomerization was proved. The isomeric transformation ${ }^{71-72}$ of hexafluorocyclobutene to hexafluorobutadiene has been observed, and the separation of the carbon isotopes was observed through such isomerization. $^{72}$

\subsubsection{Experimental results for various molecules}

We will consider below the basic experimental results on molecular isomerization by $\mathrm{CO}_{2}$ laser pulses.

2-butene. In the experiments ${ }^{67}$ with trans- and cis-isomers of 2 butene, they were irradiated with focused $\mathrm{CO}_{2}$ laser pulses. Both isomers absorb the $\mathrm{CO}_{2}$ laser radiation, the absorption factor of the 
trans-isomer is 11.5 times larger. The activation energy of cis-transisomerization is estimated to be about 20 quanta of $\mathrm{CO}_{2}$ laser radiation. In the experiments pure trans- and pure cis-isomers as well as their mixture were irradiated. When irradiated the trans-isomer gives the following products: methane, ethylene, propane, cis-2-butene and butadiene. The product distribution and the relative conversion rates depended slightly both on the pressure of parent molecules and the pressure of inert gases. The same final products were produced from irradiating the cis-isomer, and the formation of trans-isomer was observed. But the rate of formation of trans-isomer was five times lower than in the previous experiments. When the equimolar mixture of the both isomers was irradiated at a total pressure of 4 Torr $15 \%$ enrichment of the mixture with cis-isomer was obtained. This result is considered by the authors to be basic proof of the nonthermal nature of isomerization.

The results obtained may also be explained, however, by preferential dissociation of trans-2-butene due to MP absorption.

Dichlorethylene. The isomerization of trans-dichlorethylene ${ }^{68}$ to the cis-form under the action of $\mathrm{CO}_{2}$ laser pulses has been considered:<smiles>ClC=CCl</smiles>

The energy barrier of trans-cis-isomerization (Figure 7.13) equals $1.8 \mathrm{eV}$. The energy of the $\mathrm{C}=\mathrm{C}$ bond breaking in the $\mathrm{C}_{2} \mathrm{H}_{2} \mathrm{Cl}_{2}$ molecule equals $5.0 \mathrm{eV}$. The $\mathrm{CO}_{2}$ laser radiation excited the trans $-\mathrm{C}_{2} \mathrm{H}_{2} \mathrm{Cl}_{2}$ molecule whose absorption band $\nu_{6}$ lies in the region of $10.6 \mu$. The initial content of cis-isomer varied from 10 to $40 \%$ and the total pressure from 0.4 to 1.0 Torr.

As the energy fluence was over $50 \mathrm{~J} / \mathrm{cm}^{2}$ there were three processes observed at the same time: (1) reaction of trans-cis-isomerization of $\mathrm{C}_{2} \mathrm{H}_{2} \mathrm{Cl}_{2}$; (2) dissociation of trans $-\mathrm{C}_{2} \mathrm{H}_{2} \mathrm{Cl}_{2}$ yielding fragments in the ground electronic state and acetylene, the main end product; (3) dissociation yielding electronically-excited radicals $\mathrm{CH}^{*}$ and $\mathrm{C}_{2}^{*}$. Figure 7.14 shows the dependence of the relative concentrations of trans- and cis $-\mathrm{C}_{2} \mathrm{H}_{2} \mathrm{Cl}_{2}$ on the number of irradiation pulses. Even though at the beginning of irradiation the amount of cis-isomer increases, the process of trans-cis-transformation does not continue to the end. After attaining its maximum concentration of about 0.5 


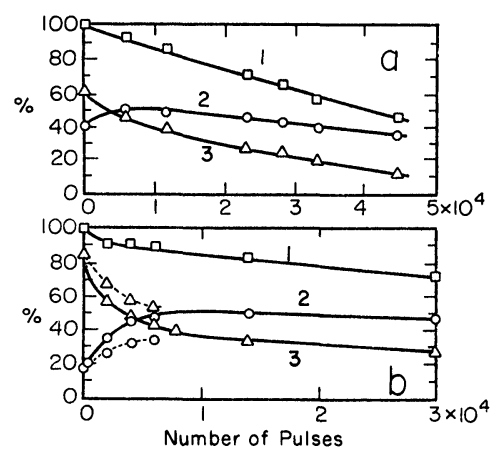

FIGURE 7.14 Dependence of the content of cis- and trans-isomers of $\mathrm{C}_{2} \mathrm{H}_{2} \mathrm{Cl}_{2}$ on the number of irradiation pulses. (1) cis +trans, (2) cis, (3) trans. (a) $\Omega=934.9 \mathrm{~cm}^{-1}$, $\Phi=100 \mathrm{~J} / \mathrm{cm}^{2}, p=0.4$ Torr. (b) $\Omega=980.9 \mathrm{~cm}^{-1}, \Phi=250 \mathrm{~J} / \mathrm{cm}^{2}, p=1.0$ Torr. The addition of 44 Torr Xe is shown with a dashed line. ${ }^{68}$

the amount of cis-isomer in the mixture begins to drop gradually. But the cis-trans concentration ratio in the mixture increases continuously and at the end of irradiation may range from 2 to 3 with the initial ratio being 0.67 (Figure 7.14a) and 0.18 (Figure 7.14b). The mixture was being enriched with cis-isomer all the time. The curves presented also illustrate the dependence of the total number of dissociated molecules on the number of pulses. It may be seen that it is mainly the trans $-\mathrm{C}_{2} \mathrm{H}_{2} \mathrm{Cl}_{2}$ molecules that dissociate.

To determine how the laser pulse tail affects the processes concerned some experiments were carried out with a "shortened" laser pulse without a tail (the duration of such a pulse was $250 \mathrm{~ns}$ with respect to base and $60 \mathrm{~ns}$ with respect to half-height). It was found that neither fluorescence nor isomerization were observed at the same intensity of IR field, but only dissociation giving unexcited products took place. When a pulse with a long tail is used all three processes occur in the same energy range. They all stop when the energy fluence drops below 50 to $100 \mathrm{~J} / \mathrm{cm}^{2}$.

Experiments with buffer gases are important for distinguishing the applicable isomerization mechanism for a particular chemical system. Adding a buffer gas produces several effects. First, a buffer gas affects rotational, vibrational and translational relaxation thus acting on the process of MP excitation. Second, as stressed above, adding a buffer gas must have a strong effect on the stabilization rate of molecules in one of the isomeric forms. It also increases the heat capacity of the 
gas mixture. All other experimental conditions being equal, this causes the maximum temperature to decrease and thus suppresses thermal processes. In this case it is only buffer gas heat capacity that is of importance. In the experiments with dichlorethylene the role of different buffer gases ( $\mathrm{Xe}, \mathrm{He}, \mathrm{H}_{2}, \mathrm{~N}_{2}$ ) was studied with the following results:

(1) With the addition of a buffer gas the absorbed energy increases. Adding 33 Torr of Xe increases the absorbed energy by more than 10 times which almost completely compensates for the increase of heat capacity. It is clear that in this case the buffer gas cannot suppress thermal processes.

(2) Buffer gases partially suppress the process of isomerization. Measurements show that the suppression effect varies for different gases at the same heat capacity. It also varies for different "buffer gas-dichlorethylene" mixtures with the same heat capacity.

(3) Buffer gas does not have a pronounced effect on the dissociation yield (see Figure 7.13).

(4) At low pressures of $\mathrm{C}_{2} \mathrm{H}_{2} \mathrm{Cl}_{2}$ (0.3 Torr) all buffer gases increase the yield of electronically excited dissociation products almost by an order of magnitude.

1,5-hexadiene. The Cope rearrangement in 1,5-hexadiene by $\mathrm{CO}_{2}$ laser radiation ${ }^{70}$ was studied:

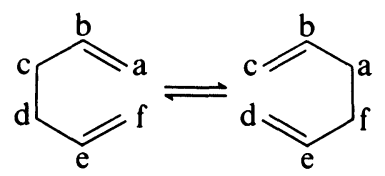

Into certain points of this molecules deuterium was substituted for hydrogen. The initial and final products are chemically the same; they have a zeropoint energy difference and differ in their vibrational spectra. This allows selective excitation of one of the forms. The activation energy of reaction (7.15) $E_{A}=12000 \mathrm{~cm}^{-1}$.

One example of the isomerization reaction of deuterated compounds ${ }^{70}$ is

(A)<smiles>[2H]C(=C)CCC([2H])=C([2H])C([2H])=C([2H])[2H]</smiles><smiles>[2H]C(=C)C([2H])([2H])C([2H])([2H])C([2H])=C</smiles>

The (A) compound: 1,5-hexadiene-1,1,2,5,6,6- $d_{6}$ was synthesized (the 
left part of (7.16)). The Cope rearrangement must give birth to the (B) compound: 1,5-hexadiene-2,3,3,4,4,5- $d_{6}$. Heating the initial (A) compound gave a mixture of the (A) and (B) compounds with their concentration ratio of 0.9 . The (A) compound is quite transparent at $10.8 \mu$ whereas the (B) compound features strong absorption at this wavelength $\left(\kappa=5.8 \cdot 10^{-3} \mathrm{~cm}^{-1} \mathrm{Torr}^{-1}\right)$. After the mixture was irradiated by $\mathrm{CO}_{2}$ laser pulses at $10.8 \mu$ the (B) compound disappeared without formation of any dissociation products. In the mixture only the (A) compound remained. The selectivity factor of the isomerization reaction was much higher than unity, and it decreased with increasing pressure. The same results were obtained in case of other deuterated 1,5-hexadiene molecules. These apparently were the first experiments in which the isomerization reaction observed had a rate much higher than the rate of dissociation reaction.

Methylisocyanide. The isomerization reaction of methylisocyanide ${ }^{69}$ by $\mathrm{CO}_{2}$ laser radiation to acetonitrile has been studied:

$$
\mathrm{CH}_{3} \mathrm{NC} \rightarrow \mathrm{CH}_{3} \mathrm{CN}
$$

The reaction barrier (7.17) equals $12500 \mathrm{~cm}^{-1} .{ }^{69}$ Isomerization of methylisocyanide was observed irradiated with $\mathrm{CO}_{2}$ laser pulses in the region of $10.6 \mu$ where the $\nu_{4}$ vibration of $\mathrm{CH}_{3} \mathrm{NC}$ lay. It was found that, with the radiation intensity over $70 \mathrm{MW} / \mathrm{cm}^{2}$, isomerization of the molecules occurred simultaneously with their dissociation, the latter followed by luminescence of the electronically excited radicals $\mathrm{CN}^{*}$ and $\mathrm{C}_{2}^{*}$. When the $\mathrm{CO}_{2}$ laser radiation intensity is lower only isomerization takes place. No direct dissociation products of $\mathrm{CH}_{3} \mathrm{NC}$ were observed.

Experiments at different pressures of $\mathrm{CH}_{3} \mathrm{NC}$ show that the isomerization yield increases as the gas pressure grows. It should be noted that isomerization of methylisocyanide was also observed at low gas pressures, up to $10^{-2}$ Torr.

Hexafluorocyclobutene. The transformation of hexafluorocyclobutene (HFCB) ${ }^{71,72}$ to hexafluorobutadiene (HFB) under $\mathrm{CO}_{2}$ laser pulses has been studied

(HFCB)<smiles>FC1=C(F)C(F)(F)C1(F)F</smiles>

(HFB)<smiles>FC(F)=C(F)C(F)=C(F)F</smiles> 
The reaction barrier (7.18) equals $16400 \mathrm{~cm}^{-1}$ and that of the reverse reaction of HFB cyclization $12200 \mathrm{~cm}^{-1}$.

In irradiating 0.5 Torr of pure $\mathrm{HFCB}^{71}$ the formation of $\mathrm{HFB}$ molecules was observed. With focused radiation the process of isomerization was also followed by the dissociation of the compound under exposure. The isomerization in a non-focused laser beam was the basic reaction but the maximum transformation of HFCB to HFB obtained under such conditions was no higher than $60 \%$ which $^{71}$ was due to the equilibrium established during the process of irradiation between the direct reaction (7.18) and the reverse reaction of cyclization.

Adding a buffer gas $(\mathrm{He})$ radically changes the course of the reaction. Although at the very beginning of irradiation the isomerization yield of reaction (7.18) decreases, the reverse reaction is suppressed to a greater degree. At a helium pressure of 16 Torr, the reverse reaction was suppressed fully. As a result, the isomerization yield in each pulse during the entire time of irradiation was constant. Therefore, in the presence of the buffer gas it was possible to practically fully convert HFCB to HFB under the action of $\mathrm{CO}_{2}$ laser pulses.

Carbon isotope separation ${ }^{72}$ has been performed using reaction (7.18). By choosing a proper frequency of $\mathrm{CO}_{2}$ laser radiation it was possible to establish preferential conditions of reaction (7.18) for the molecules containing only ${ }^{12} \mathrm{C}$ or also ${ }^{13} \mathrm{C}$ atoms. As a result, the HFB molecules were enriched with the ${ }^{12} \mathrm{C}$ or ${ }^{13} \mathrm{C}$ isotopes respectively. At the $10.64 \mu$ wavelength, for example, the resulting HFB molecules were enriched with the ${ }^{13} \mathrm{C}$ isotope. With the initial pressure of $\mathrm{HFCB}$ 0.5 Torr and the He pressure 16 Torr, the isomerization yield of the $\mathrm{HFCB}$ molecules containing ${ }^{13} \mathrm{C}$ was $\beta_{13}=6.4 \cdot 10^{-4}$ and was 12.8 times higher than the isomerization yield $\beta_{12}$ of the molecules containing only ${ }^{12} \mathrm{C}$.

As the HFCB molecules were irradiated at $10.3 \mu$, preferential isomerization of the molecules containing only ${ }^{12} \mathrm{C}$ was observed and consequently the HFB molecules were enriched with the ${ }^{12} \mathrm{C}$ isotope.

It is worthy of notice that the isotopically selective isomerization of HFCB took place only at a comparatively low pressure of these molecules ( 0.5 to 1.0 Torr) and with the addition of a buffer gas. In the absence of a buffer it was impossible to achieve isotopically selective isomerization. 


\subsubsection{Mechanisms of IR laser-induced isomerization}

There are several potential mechanisms which may be responsible for molecular isomerization in a strong IR field (see Figure 7.13).

(1) Multiphoton excitation of the high-lying vibrational levels of a molecule with the result that the molecules acquire energy exceeding the isomerization barrier. If the stored energy is localized at the degree of freedom responsible for isomerization the structural form of the molecule is subjected to changes. This mechanism employs molecular excitation only within the ground electronic state.

(2) Excitation of the electronic state of the molecule due to vibrational energy transfer, i.e., the $V \rightarrow E$ process, with subsequent rearrangement of the molecule in the excited electronic state. In this case the isomerization mechanism is a usual one (see Ref. 66), the only difference consisting in a new way of electronic state excitation.

(3) Selective dissociation of the molecular isomeric form due to MP excitation. The mixture can be enriched with one of the isomers by dissociating the other one. In this case the molecular concentration of the unexcited isomer (for example, cis $-\mathrm{C}_{2} \mathrm{H}_{2} \mathrm{Cl}_{2}$ ) may also increase due to recombination of the radicals formed by dissociation of trans$\mathrm{C}_{2} \mathrm{H}_{2} \mathrm{Cl}_{2}$ resulting in all possible isomeric forms. This mechanism causes one isomer to burn out and the others to accumulate.

(4) Thermal isomerization due to an increase of temperature in the region under irradiation after the absorbed energy is thermalized. Such a process is nonselective, and a strong IR field is necessary only to provide a high level of absorbed energy.

In all the above experiments on molecular isomerization by IR radiation-pulses the thermal mechanism has been eliminated. The most direct proof of the nonthermal nature of the mechanism is observation of the considerable enrichment of the mixture with one of the isomers to levels exceeding their equilibrium concentration. In the experiments with $\mathrm{C}_{2} \mathrm{H}_{2} \mathrm{Cl}_{2}$, for example, the ratio between the initial and final ratios of trans- and cis-forms was 10 . By varying the radiation frequency it was possible to reverse the ratio (from the initial one- 0.18 to the final one-2).

Observation of isotopic selectivity in isomerization of HFCB molecules is also direct proof of the nonthermal mechanism of isomerization. The simple estimations of the temperature conditions after the pulse action in these experiments also reject thermal isomerization. In the experiments with $\mathrm{C}_{2} \mathrm{H}_{2} \mathrm{Cl}_{2}$, for example, the temperature 
in the zone under irradiation was no higher than $650 \mathrm{~K}$. The thermal isomerization time at this temperature ${ }^{66}$ is about $10^{3} \mathrm{~s}$, and the characteristic time of its cooling down was $10^{-4}$ to $10^{-5} \mathrm{~s}$. This excludes the possibility of any essential contribution of the thermal mechanism of $\mathrm{C}_{2} \mathrm{H}_{2} \mathrm{Cl}_{2}$ isomerization. This conclusion can be also applied to other molecules.

It is difficult to choose between the other selective mechanisms $1^{\circ}$ to $3^{\circ}$. In the experiments with 1,5 -hexadiene ${ }^{70}$ the absence of appreciable dissociation enables us to turn down recombination-dissociation mechanism $3^{\circ}$. This also applies to the experiments with methylisocyanide $^{69}$ and hexafluorocyclobutene ${ }^{71-72}$ where, at least under some experimental conditions, only isomerization reaction took place. It is difficult, however, to make an unambiguous choice between possible mechanisms $1^{\circ}$ and $2^{\circ}$ without additional experiments which would define the nature of the molecular states excited by $\mathrm{CO}_{2}$ laser radiation. It is particularly important to measure the average level of excitation of the molecules interacting with the radiation.

In the experiments ${ }^{68}$ with dichlorethylene the threshold energy (or power) for the processes of dissociation, isomerization and luminescence was the same. This points to the fact that the contribution of mechanisms $2^{\circ}$ and $3^{\circ}$ may be essential. In case of $\mathrm{C}_{2} \mathrm{H}_{2} \mathrm{Cl}_{2}$ one more rather specific isomerization channel is possible. If the dissociation of these molecules gives birth to atomic chlorine, which is quite probable since the $\mathrm{C}_{2}$ and $\mathrm{CH}$ radicals are identified from the luminescence spectra, ${ }^{68}$ the atoms of chlorine in this case can induce a chain reaction through the formation of vibrationally excited radicals $\mathrm{C}_{2} \mathrm{H}_{2} \mathrm{Cl}_{3}^{*}:^{73}$

$$
\mathrm{Cl}+\text { trans }-\mathrm{C}_{2} \mathrm{H}_{2} \mathrm{Cl}_{2}
$$

The $\mathrm{C}_{2} \mathrm{H}_{2} \mathrm{Cl}_{3}^{*}$ radicals quickly dissociate to trans- or cisdichlorethylene with detachment of the $\mathrm{Cl}$ atom which continues the chain. As the experiments show, ${ }^{74}$ the length of this chain may be very significant (about $10^{3}$ ). Thus, even at small amounts of resultant $\mathrm{Cl}$ the contribution of this process may be great.

Once again we want to stress the necessity of fast stabilization of molecules in one of the isomeric forms after they have acquired energy higher than the isomerization barrier in the proces of MP excitation. 
This has been vividly demonstrated by experiments on isomerization of HFCB. ${ }^{72}$ The isotopic selectivity of this process was observed only with adding a buffer gas. The collisions with the buffer gas enable the excited molecules to give away the energy excess and prevent $V-V$ exchange with other molecules thus preserving laser excitation selectivity.

\subsection{Role of collisional processes in IR multiple photon dissociation}

The results of studying the excitation and dissociation of molecules by IR radiation discussed in the foregoing sections have been obtained on the assumption that the effect of collisions on the process of excitation may be neglected. In most cases this was achieved by choosing a rather low gas pressure so that the laser pulse duration could be shorter than the time between two successive gas-kinetic collisions $\tau_{p}<\tau_{\text {coll }}$. The stringency of this condition is discussed below. When this condition is not fulfilled collisions can affect the character of excitation of molecules in the IR field and their subsequent dissociation.

Studying the role of collisional processes is important from several standpoints. First, it allows obtaining additional important information on the mechanism of excitation and dissociation of an isolated molecule. Secondly, experiments of this kind are of special interest to us since they make it possible to study the relaxation processes in highly vibrationally excited molecules.

The experimental results below show how the collisions of molecules both between themselves and with buffer gases affect the dissociation yield. Information on secondary chemical processes following molecular dissociation and the effect they can produce on the measurement of MP dissociation parameters will also be discussed.

Questions concerning the effect of collisions on the selectivity of MP excitation and dissociation are considered in Chapter 8.

\subsubsection{Collisions with a buffer gas}

The collisions of the excited molecules with the buffer gas may give rise to several relaxation processes whose effect on MP excitation and dissociation of molecules is schematically shown in Figure 5.18. First, the rotational state of the molecule in the ground or excited vibrational 
state can be changed. The effect of rotational relaxation at the initial stage of MP excitation was considered in detail in Section 5.3.1. We will just recall that this process cannot change substantially the molecular energy but it can involve new molecules into the process of MP excitation populating the resonant states devastated by the field. This, of course, brings about an increase in dissociation yield if a small fraction of molecules $q$ takes place originally in MP excitation (see Figure 5.18). The characteristic pressures, at which the rotational relaxation caused by collisions with a small (few-atom) buffer gas begins to have an effect on the process of MP excitation, range approximately from 0.1 to 1 Torr at $\tau_{p} \simeq 10^{-7} \mathrm{~s}$.

Secondly, during collisions with the buffer gas the vibrational state of the molecule can be changed. As a result, the energy is transferred to other degrees of freedom (vibrational, rotational, translational) of the molecule itself or the buffer gas. Therefore, as opposed to rotational relaxation, the $V-V$ and $V-T / R$ processes decrease the vibrational energy of excited molecules and hence the dissociation yield. The influence of rotational and $V-T / R$ relaxation on the MP dissociation process is rather well seen from Figure 7.15, where the dependence of $\mathrm{CF}_{3} \mathrm{D}$ dissociation yield on Ar pressure is presented. Initial growth of $\beta$ with increasing Ar pressure is caused by new molecules involved in the process of MP excitation due to rotational relaxation. ${ }^{103}$ Subsequent decrease in $\beta$ is a result of $\mathrm{CF}_{3} \mathrm{D}$ deexcitation due to $V-T / R$ relaxation. It must be said that the effect of molecular deexcitation takes place both during a laser pulse and after it. The latter should be always kept in mind since the fulfillment of the condition of collisionless MP excitation $\tau_{p}<\tau_{\text {coll }}$ does not guarantee the collisionless character of the entire process of MP dissociation.

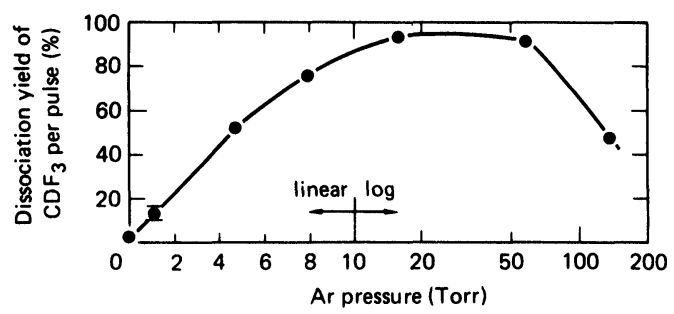

FIGURE 7.15 Dependence of the dissociation yield of $\mathrm{CF}_{3} \mathrm{D}$ on $\mathrm{Ar}$ pressure. The pressure of $\mathrm{CF}_{3} \mathrm{D}$ is $6.5 \cdot 10^{-2}$ Torr. $\Phi=25 \mathrm{~J} / \mathrm{cm}^{2} .{ }^{103}$ 
The real contribution of each of the relaxation processes listed $\dagger$ depends on many factor. It is determined by the parameters of the molecule under excitation, the conditions of its excitation, the type of buffer gas and its pressure.

The role of rotational relaxation was considered in Section 5.3, and so in this section we are going to dwell on the contribution of vibrational deexcitation caused by the $V-T$ process. In Section 2.5.6 it was noted that the typical value of $V-T$ relaxation rate $k_{V-T} \simeq$ $10^{3}-10^{5} \mathrm{~s}^{-1} \mathrm{Torr}^{-1}$. This value of $k_{V-T}$, however, corresponds to small excitation levels of molecules when the relaxing system slightly deviates from equilibrium. At strong excitation of molecules, when they are in the quasi-continuum the $V-T$ process cannot be described only by the rate constant $k_{V-T}$ because it also begins to depend on molecular energy ${ }^{75}$ In describing the relaxation of highly excited molecules it is more convenient to apply another parameter, that is, the average energy $\langle\Delta E\rangle$ transferred in one gas-kinetic collision. The value $\langle\Delta E\rangle$ depends on the type of colliding partners, and in case of highly excited molecules it usually varies between 150 and $1000 \mathrm{~cm}^{-1} .{ }^{76}$ As the number of atoms in a buffer gas molecule increases the $\langle\Delta E\rangle$ value tends to increase. For example, in case of deexcitation of cycloheptatriene with $\mathrm{C}_{2} \mathrm{~F}_{6}$ molecules the $\langle\Delta E\rangle$ value comes to $1700 \mathrm{~cm}^{-1}$. $^{76}$

Let us estimate now the pressure at which $V-T$ relaxation must affect the MP excitation of molecules in the quasi-continuum. It is obvious that this takes place when the rate of energy outflow caused by the $V-T$ process is comparable with the rate of its inflow $d E / d t$ into the molecule by MP absorption, i.e., when

$$
\frac{d E}{d t} \simeq\langle\Delta E\rangle Z_{0} p
$$

where $Z_{0}$ is the frequency of gas-kinetic collisions, $p$ is the buffer gas pressure. Usually $Z_{0} \simeq 107 \mathrm{~s}^{-1} \mathrm{Torr}^{-1}$. For rough estimation we take $d E / d t=D / \tau_{p}$. From (7.19) it is easily obtained that for $D=$ $(20-40) \cdot 10^{4} \mathrm{~cm}^{-1}$ and $\tau_{p}=(1-2) \cdot 10^{-7} \mathrm{~s}$ the buffer gas pressure at which the vibrational deexcitation becomes essential ranges from ten to several hundreds of Torr. The experimental results agree with this estimate.

\footnotetext{
$\dagger$ Collisions may also disturb the coherence of MP excitation if it takes place. See Chapter 5.
} 
For illustration, let us consider the effect of vibrational deexcitation on the process of MP excitation and dissociation of $\mathrm{SF}_{6}$ that has been quite studied in detail. Figure 7.16 shows the dependence of the dissociation yield $\beta$ of $\mathrm{SF}_{6}$ on the pressure of some buffer gases. Ar and such molecular gases as $\mathrm{O}_{2}, \mathrm{C}_{2} \mathrm{H}_{4}$ and $\mathrm{NH}_{3}$ were used as buffers. It may be seen that in the pressure range under study for all gases there is an exponential decrease of $\beta$ with increasing pressure. The rate of this decrease can be characterized, for example, by the value $P_{\text {buf }}(1 / e)$, i.e., by the pressure at which $\beta$ drops by $e$ times. As seen from Figure 7.16, the value $P_{\text {buf }}(1 / e)$ depends on the type of buffer gas. A stronger dependence on the pressure of $\mathrm{NH}_{3}$ and $\mathrm{C}_{2} \mathrm{H}_{4}$, as compared to $\mathrm{O}_{2}$ and $\mathrm{Ar}$, is explained by resonant energy transfer in the first case brought about by the $V-V$ process since the $\mathrm{NH}_{3}$ and $\mathrm{C}_{2} \mathrm{H}_{4}$ molecules have vibrations close in frequency to the $\nu_{3}$ vibration of the $\mathrm{SF}_{6}$ molecule. A decrease in dissociation yield with the addition of a buffer gas was observed in all experiments of this kind. Even though at first there is an increase of $\beta$ caused by the effect of rotational relaxation, the effect of vibrational deexcitation, starting from some pressure, becomes dominant (see Figure 7.15). As in $\mathrm{SF}_{6}$, in most cases the decrease in $\beta$ is exponential or almost exponential. The difference lies only in the value $P_{\text {buf }}(1 / e)$. For example, $\mathrm{SF}_{6}$ (Figure 7.16) in the presence of $\mathrm{Ar} P_{\text {buf }}(1 / e) \simeq 12-15$ Torr, for $\mathrm{CF}_{2} \mathrm{HCl}$ mixed with $\mathrm{Ar}$ $P_{\text {buf }}(1 / e) \simeq 200$ Torr $^{77}$ and for $\mathrm{CF}_{3} \mathrm{Br}$ with $\mathrm{N}_{2} P_{\text {buf }}(1 / e) \simeq 400$ Torr. $^{78}$

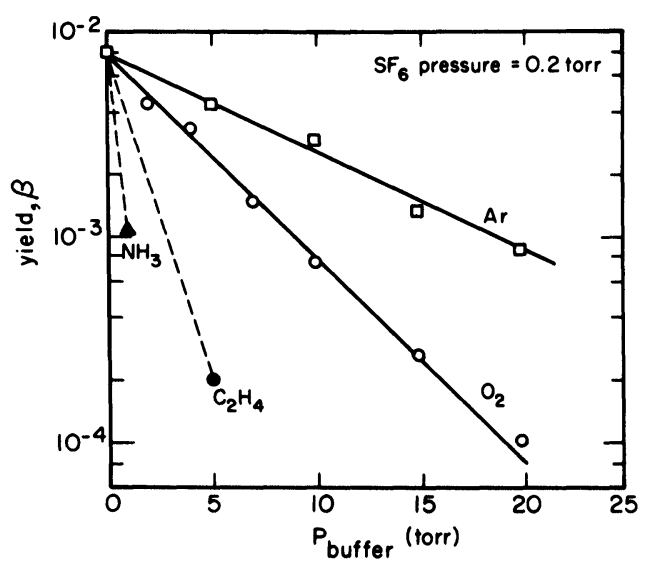

FIGURE 7.16 Dependence of the dissociation yield of $\mathrm{SF}_{6}$ on the buffer gas pressure. ${ }^{19}$ 
Such a difference is evidently caused by different values of the $\langle\Delta E\rangle$ parameter as well as by different excitation rates of these molecules. The latter can be clearly seen in experiments with $\mathrm{SF}_{6}$ mixed with Ar. The results presented in Figure 7.16 are obtained with $\Phi=2.5 \mathrm{~J} / \mathrm{cm}^{2}$, i.e., with a small excess of dissociation limit. The increase of radiation energy fluence up to $\Phi=5.1 \mathrm{~J} / \mathrm{cm}^{2}$ increases $P_{\text {buf }}(1 / e)$ almost by 2.5 times, approximately up to 40 Torr. $^{79}$ Comparing the experimental dependence $\beta=\beta$ ( $p_{\text {buf }}$ ) with those calculated by the model it is possible to find a number of excitation parameters including the value of $\langle\Delta E\rangle$. In the case of $\mathrm{CF}_{2} \mathrm{CFCl}$ mixed with $\mathrm{Ar}^{80}$ such comparison gives $\langle\Delta E\rangle \simeq 900 \mathrm{~cm}^{-1}$ which is consistent with typical values of this parameter.

At a very high pressure of buffer gas, when the inequality $d E / d t \ll$ $Z_{0}\langle\Delta E\rangle p$ is fulfilled, high MP excitation of molecules becomes impossible. The absorbed energy is transferred "instantaneously" to the buffer gas, and in this case a purely two-level excitation scheme can be realized. This effect for $\mathrm{SF}_{6}$ has been observed with $I \simeq 10^{7} \mathrm{~W} / \mathrm{cm}^{2}$ and helium pressure $20-40 \mathrm{~atm}^{81}$

The collisions of the molecules with a buffer affect not only their MP excitation during a laser pulse. The deexcitation after the pulse is over may also be an essential factor. This process is especially important for large molecules characterized by a long time of unimolecular dissociation $\tau_{\text {diss }} \gg \tau_{p}$. So their deexcitation after the pulse is over is a decisive factor. To confirm this conclusion Figure 7.17 shows the dependence of the dissociation yield $\beta$ for $\mathrm{CF}_{3} \mathrm{Br}$ and $\left(\mathrm{CF}_{3}\right)_{3}$ $\mathrm{CBr}$ on the $\mathrm{I}_{2}$ pressure. ${ }^{82}$ These molecules have almost the same energies of breaking of the $\mathrm{C}-\mathrm{Br}$ bond $\left(D \simeq 25000 \mathrm{~cm}^{-1}\right)$. In the experiment with the $\mathrm{CF}_{3} \mathrm{Br}$ molecule the average absorbed energy was

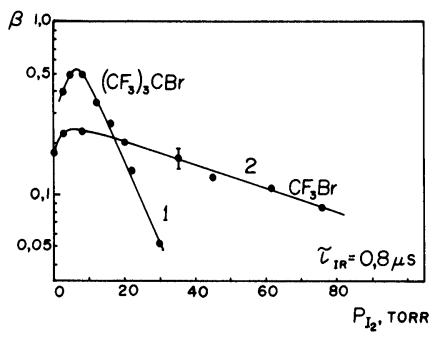

FIGURE 7.17 Dependence of the dissociation yield of $\left(\mathrm{CF}_{3}\right)_{3} \mathrm{CBr}$ (curve 1) and $\mathrm{CF}_{3} \mathrm{Br}$ (curve 2) on $\mathrm{I}_{2}$ pressure ${ }^{82} \tau_{p}=8 \cdot 10^{-7} \mathrm{~s}$. The initial increase in curves 1 and 2 is connected with recombination suppression. 
$\bar{\varepsilon} \leqslant D$ and the dissociation basically occurred within a laser pulse. The situation differed for the $\left(\mathrm{CF}_{3}\right)_{3} \mathrm{CBr}$ molecule when considerable overexcitation took place. The molecules dissociated in a longer time. With $E_{\mathrm{vib}}-D=4 \cdot 10^{4} \mathrm{~cm}^{-1}$, for example, $\tau_{\text {diss }}=10^{-4} \mathrm{~s}$, and therefore the main contribution to deexcitation is given by collisions after MP excitation. Because of this the dependence of $\beta$ on buffer gas pressure for these molecules is much steeper despite the MP excitation rate being almost twice as much for $\left(\mathrm{CF}_{3}\right)_{3} \mathrm{CBr}$. The value of $P_{\text {buf }}(1 / e)$ in this case is almost an order less than that for $\mathrm{CF}_{3} \mathrm{Br}$.

The results of a great number of experiments show that the collisions between the excited molecules and the buffer gas can importantly affect the process of MP dissociation. There are two types of dependence of $\beta$ on buffer gas pressure (Figure 5.18). When the radiation interacts with almost all the molecules and $q=1\left(\mathrm{SF}_{6}, \mathrm{CF}_{3} \mathrm{I}\right)$ the role of rotational relaxation is modest, and the addition of the buffer gas only causes $\beta$ to decrease due to vibrational deactivation. When $q \ll 1$, with addition of the buffer gas $\beta$ increases at first due to rotational relaxation, and only then vibrational deactivation begins to manifest itself.

In conclusion it should be noted that, with a buffer gas added, there may be also a decrease in total heating of the gas after the absorbed energy is thermalized. This, in its turn, can have influence on the course of secondary chemical reactions (see Section 7.4.3).

\subsubsection{Collisions between excited molecules}

When the excited molecules collide with each other we can observe, of course, the same processes which take place as they collide with the buffer gas (rotational relaxation and vibrational energy deactivation). Furthermore, a resonant process of $V-V$ exchange can take place

$$
M(v=n)+M(v=m) \rightarrow M(v=n+1)+M(v=m-1)+\Delta E^{\prime}
$$

This process, unlike the case of collisions with the buffer gas, does not bring about a decrease in the average vibrational energy of molecules since the defect of energy $\Delta E^{\prime}$ is usually small. Exchange (7.20), however, can affect the process of molecular excitation, particularly at its initial stage, when the radiation intensity, for example, does not suffice to excite the molecules to the quasi-continuum. In this case the molecules can reach the quasi-continuum limit due to 
energy exchange by scheme (7.20), and then they can be effectively excited by IR radiation. Such a process was applied ${ }^{83}$ to explain experiments on dissociation of $\mathrm{SiH}_{4}$ molecules by pulsed IR radiation. In the case of $\mathrm{SiH}_{4}$ the dissociation threshold increased as the gas pressure was decreased. It was impossible to cause dissociation of $\mathrm{SiH}_{4}$ under collisionless conditions with the radiation energy fluence up to $\Phi=150 \mathrm{~J} / \mathrm{cm}^{2}$. As a result of $V-V$ exchange at the first stage of excitation the dissociation yield of $\mathrm{SiH}_{4}$ significantly depends on its pressure and increases as the latter rises. The same situation takes place in the dissociation of $\mathrm{CH}_{3} \mathrm{CN}$ molecules ${ }^{25}$ in the collisional regime.

For a lot of molecules the dissociation yield is independent of their own pressure. Thorough measurements for $\mathrm{SF}_{6}{ }^{84,2}$ when the recombination was suppressed show that the dissociation yield does not depend on the pressure in the range from 0.06 to 3.75 Torr. The same results were obtained ${ }^{85}$ for $\mathrm{CH}_{3} \mathrm{OH}$ with the pressure increased up to 10 Torr. This can be explained if the fraction of molecules excited to the quasi-continuum $q \simeq 1$ so that the $V-V$ exchange with the cold ensemble is negligible.

But even when all the molecules are in the hot ensemble their relaxation from the nonstationary distribution to the Boltzmann one may change the number of molecules over the dissociation limit and, as a result, change the dissociation yield. This process becomes particularly significant after the laser pulse. As early as in the very first works on MP dissociation of $\mathrm{SiF}_{4}{ }^{1}$ and $\mathrm{BCl}_{3}{ }^{2}$ the collisional "phase" of dissociation was explained by collisions of highly excited molecules.

Another important consequence of resonant $V-V$ exchange is the loss of selectivity of MP excitation and dissociation. $V-V$ exchange leads to energy transfer from the excited molecules, for example with a particular isotope, to the nonexcited molecules with a different isotope. As a result, these molecules reach the quasi-continuum and then may be excited up to the dissociation limit by IR radiation. The influence of $V-V$ exchange on the selectivity of MP dissociation will be discussed in detail in Chapter 8 .

\subsubsection{Secondary chemical reactions}

The fragments of MP dissociation in most cases are atoms and radicals. These fragments are not chemically stable, therefore subsequent col- 
lisions lead to the formation of the final stable molecules. $\dagger$ In some cases these stable molecules may be the initial substance. To suppress this recombination an additional reagent acting as a scavenger of the radicals formed by dissociation is usually introduced into the gas under irradiation.

In some cases recombination is a dominant process, for example in dissociation of $\mathrm{BCl}_{3},{ }^{87}$ and the expenditure of the gas occurs only in the presence of a scavenger. In other cases the contribution of recombination is not so great, and the addition of a scavenger brings about only some increase of the measured dissociation yield. The role of recombination is determined by the ratio of rates of competing processes-recombination and reactions giving rise to products differing from the original substance. So, by adding a sufficient amount of scavenger it is always possible, in principle, to completely suppress recombination.

The role of secondary chemical processes has been studied in more detail in experiments on dissociation of $\mathrm{SF}_{6}$. The decrease of $\mathrm{SF}_{6}$ in the cell under irradiation was observed both in the presence and the absence of scavenger. The latter made it possible to conclude ${ }^{88}$ that the dissociation of $\mathrm{SF}_{6}$ was irreversible. The subsequent experiments, ${ }^{89}$ however, show that in the cell containing only $\mathrm{SF}_{6}$, after irradiation, the content of $\mathrm{SF}_{6}$ will return with time. With a scavenger added into the cell, such chemical reduction does not occur. Analysis of the end products of dissociation of pure $\mathrm{SF}_{6}$ shows ${ }^{83}$ that they are $\mathrm{SOF}_{2}, \mathrm{SOF}_{4}$ and $\mathrm{SO}_{2} \mathrm{~F}_{2}$ oxifluorides. These compounds are formed by the reactions of $\mathrm{SF}_{5}$ and $\mathrm{SF}_{4}$ arising in dissociation of $\mathrm{SF}_{6}$ with the water adsorbed on the cell walls. Thus, the measured dissociation yield of $\mathrm{SF}_{6}$ may materially depend on the condition of the cell walls. It has been shown ${ }^{80}$ that in irradiating pure $\mathrm{SF}_{6}$, with the cell walls thoroughly cleaned, there was no expenditure of $\mathrm{SF}_{6}$ observed. Under the same conditions but with addition of $\mathrm{H}_{2}$ as a scavenger the dissociation yield was $\beta=0.15$. These experiments point to the fact that the fragments formed by dissociation of $\mathrm{SF}_{6}$ are unstable and when there is no scavenger their full recombination to the original molecule is possible.

† The formation of ions was also observed in this type of secondary reactions of MP dissociation fragments (Section 7.8.6). 
When a scavenger is added to the gas it should be taken into account that the scavenger can simultaneously act as neutral buffer gases and in a proper way affect both the process of molecular excitation and their subsequent dissociation. The introduction of a scavenger, in particular, can bring about partial deexcitation of excited molecules as illustrated by experiments with $\mathrm{CF}_{3} \mathrm{I} \mathrm{I}^{91,92}$ The dissociation of this molecule by $\mathrm{CO}_{2}$ laser radiation is followed by detachment of the iodine atom. ${ }^{91}$ Subsequent reactions cause some part of radicals, around $40 \%,{ }^{91}$ in the absence of scavenger to turn to original $\mathrm{CF}_{3} \mathrm{I}$ as a result of recombination. The rest form the final product $\mathrm{C}_{2} \mathrm{~F}_{6}$. The addition of the $\mathrm{O}_{2}$ as a scavenger increases the dissociation yield as is shown by curve 1 in Figure 7.18. But at the same time the addition of $\mathrm{O}_{2}$ leads to deactivation of $\mathrm{CF}_{3} \mathrm{I}$ which leads to the subsequent decrease of $\beta$. This is confirmed by the results of the experiment with the neutral buffer gas $\mathrm{N}_{2}$ where a monotonous decrease of $\beta$ can be observed at once (curve 2, Figure 7.18). The experiment with $\mathrm{CF}_{3} \mathrm{I}$ allowed taking into account the contribution of deactivation and determining the primary value of $\beta$. These results show that in experiments with scavengers it is always possible to determine their influence on excitation and subsequent dissociation of molecules.

It is well known that the rate of many chemical reactions is greatly affected by the temperature of the medium. This, accordingly, must affect the character of secondary chemical processes. In the aboveconsidered case of $\mathrm{CF}_{3} \mathrm{I}$, for example, the reduction in initial gas temperature caused displacement of the equilibrium towards recombination. ${ }^{92}$ The gas heating, after the laser pulse has passed, which is determined by the value of absorbed energy may result in thermal dissociation of some compounds. For example, it has been concluded ${ }^{39}$

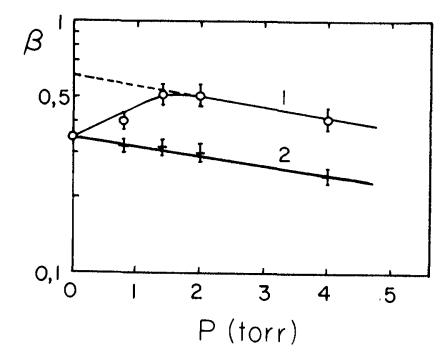

FIGURE 7.18 Dependence of the dissociation yield of $\mathrm{CF}_{3} \mathrm{I}$ on the pressure of $\mathrm{O}_{2}$ (curve 1) and $\mathrm{N}_{2}$ (curve 2). ${ }^{92}$ 
that in dissociation of $\mathrm{CF}_{2} \mathrm{Cl}_{2}$ a part of $\mathrm{CF}_{2}$ radicals can be formed by thermal dissociation of $\mathrm{CF}_{2} \mathrm{Cl}$ another primary fragment.

Studies into secondary chemical reactions and analysis of their end products with the addition of different scavengers enable in some cases drawing a conclusion on primary products of molecular dissociation. We can refer particularly to experiments with $\mathrm{CH}_{3} \mathrm{OH},{ }^{85} \mathrm{C}_{2} \mathrm{H}_{4},{ }^{94}$ $\mathrm{SF}_{5} \mathrm{Cl},{ }^{95}$ etc. In Ref. 39 such studies made it possible to estimate the contribution of each of the two dissociation channels for the $\mathrm{CF}_{2} \mathrm{Cl}_{2}$ molecule-detachment of the chlorine atom or molecule.

It should be noted that the understanding of secondary chemical processes is important not only for estimating the true primary characteristics of MP dissociation. The study of these proceses has another important aspect. With proper choice of scavengers and excitation conditions it is possible to control chemical processes and produce wanted end products. This is important for the practical application of the effect of MP dissociation and particularly for isotope separation and chemical synthesis.

\section{References}

1. N. R. Isenor, V. Merchant, R. S. Hallsworth and M. C. Richardson, Canad. J. Phys. 51, 1281 (1973).

2. R. V. Ambartzumian, N. V. Chekalin, V. S. Doljikov, V. S. Letokhov and E. A. Ryabov, Chem. Phys. Lett. 25, 515 (1974).

3. R. V. Ambartzumian, Yu. A. Gorokhov, V. S. Letokhov and G. N. Makarov, $Z h$. Eksp. Teor. Fiz. (Russian) 69, 1956 (1975) [Sov. Phys. JETP 42, 993 (1975)].

4. M. J. Coggiola, P. A. Schulz, Y. T. Lee and Y. R. Shen, Phys. Rev. Lett. 38, 17 (1977).

5. F. Brunner, T. P. Cotter, K. L. Kompa and D. Proch, J. Chem. Phys. 67, 1547 (1977).

6. R. V. Ambartzumian, N. V. Chekalin, V. S. Letokhov and E. A. Ryabov, Chem. Phys. Lett. 36, 301 (1975).

7. R. V. Ambartzumian, G. N. Makarov and A. A. Puretzky, Pis'ma Zh. Eksp. Teor. Fiz. (Russian) 28, 696 (1978) [Sov. Phys. JETP Lett. 28, 647 (1978)].

8. V. S. Doljikov, V. N. Lokhman, N. V. Chekalin and A. N. Shibanov, Kvantovaya Electron. (Russian) 5, 648 (1978).

9. Z. Karny, A. Gupta, R. N. Zare, S. T. Lin, J. Nieman and A. M. Ronn, Chem. Phys. 37, 15 (1979).

10. R. V. Ambartzumian, G. N. Makarov and A. A. Pyretzky, Appl. Phys. 22, 77 (1980).

11. O. N. Avatkov, V. N. Bagratashvili, I. N. Knyazev, Yu. R. Kolomiisky, V. S. Letokhov, V. V. Lobko and E. A. Ryabov, Kvantovaya Electron. (Russian) 4, 741 (1977).

12. V. M. Akulin, S. S. Alimpiev, N. V. Karlov, A. M. Prokhorov, B. G. Sartakov and E. M. Khokhlov, Pis'ma Zh. Eksp. Teor. Fiz. (Russian) 25, 428 (1977).

13. J. D. Campbell, G. Hancock, J. B. Halpern and K. H. 44, 404 (1976).

Chem. Phys. Lett. 
14. R. V. Ambartzumian, V. S. Letokhov, E. A. Ryabov and N. V. Chekalin, Pis'ma Zh. Eksp. Teor. Fiz. (Russian) 20, 597 (1974) [Sov. Phys. JETP Lett. 20, 273 (1974)].

15. J. M. Preses, R. E. Weston, Jr. and G. W. Flynn, Chem. Phys. Lett. 48, 425 (1977).

16. C. R. Quick, Jr. and C. Wittig, Chem. Phys. Lett. 48, 420 (1977).

17. C. R. Quick, Jr. and C. Wittig, Chem. Phys. 32, 75 (1978).

18. C. R. Quick, Jr. and C. Wittig, J. Chem. Phys. 69, 4201 (1978).

19. R. V. Ambartzumian, Yu. A. Gorokhov, V. S. Letokhov, G. N. Makarov and A. A. Puretzky, Zh. Eks. Teor. Fiz. (Russian) 71, 440 (1976) [Sov. Phys. JETP 44, 231 (1976)].

20. J. G. Black, P. Kolodner, M. J. Shultz, E. Yablonovitch and N. Bloembergen, Phys. Rev. 19A, 404 (1979).

21. Yu. R. Kolomiisky, A. R. Kukudjanov and E. A. Ryabov, Kvantovaya Electron. (Russian) 7, 1499 (1980).

22. W. Fuss, Chem. Phys. 36, 135 (1979).

23. A. Galli, Lett. Nuove Cim. 21, 357 (1978).

24. S. Speiser and J. Jortner, Chem. Phys. Lett. 44, 399 (1976).

25. V. N. Lokhman, N. V. Chekalin and A. N. Shibanov, Kvantovaya Electron. (Russian) 6, 1911 (1979).

26. D. M. Brenner, Chem. Phys. Lett. 57, 357 (1978).

27. F. M. Lussier, J. I. Steinfeld and T. F. Deutsch, Chem. Phys. Lett. 58, 277 (1978).

28. N. Bloembergen and E. Yablonovitch, in: Laser Spectroscopy III, Springer Series in Optical Sciences, Vol. 7, eds. J. L. Hall and J. L. Carlsten (Springer-Verlag, Berlin, Heidelberg, New York, 1977) p. 86.

29. V. N. Bagratashvili, V. S. Doljikov, V. S. Letokhov and E. A. Ryabov, in: LaserInduced Processes in Molecules, Springer Series in Chemical Physics, Vol. 6, eds. K. L. Kompa and S. D. Smith (Springer-Verlag, Berlin, Heidelberg, New York, 1979, p. 179).

30. R. V. Ambartzumian, Yu. A. Gorokhov, G. N. Makarov, A. A. Puretzky and N. P. Furzikov, Chem. Phys. Lett. 45, 231 (1977).

31. V. Yu. Baranov, E. P. Velikhov, Yu. R. Kolomiisky, V. S. Letokhov, V. G. Niz'ev, V. D. Pismenny and E. A. Ryabov, Kvantovaya Electron. (Russian) 6, 1062 (1979).

32. V. N. Bagratashvili, V. S. Doljikov, V. S. Letokhov, A. A. Makarov, E. A. Ryabov and V. V. Tyakht, Zh. Eksp. Teor. Fiz. (Russian) 77, 2238 (1979).

33. R. V. Ambartzumian, Yu. A. Gorokhov, V. S. Letokhov, G. N. Makarov, A. A. Puretzky and N. P. Furzikov, Pis'ma Zh. Eksp. Teor. Fiz. (Russian) 23, 217 (1976) [Sov. Phys. JETP Lett. 23, 194 (1976)].

34. M. C. Gower and T. K. Gustafson, Sov. Phys. Optics Commun. 23, 69 (1977).

35. R. V. Ambartzumian, V. S. Letokhov, G. N. Makarov and A. A. Puretzky, Optics Commun. 25, 69 (1978).

36. R. V. Ambartzumian, N. P. Furzikov, Yu. A. Gorokhov, V. S. Letokhov, G. N. Makarov and A. A. Puretzky, Optics Commun. 18, 517 (1976).

37. A. V. Baklanov, A. K. Petrov and Yu. N. Molin, Doklady AN SSSR (Russian) 242, 633 (1978).

38. J. W. Hadgens, J. Chem. Phys. 68, 777 (1978).

39. G. Folcher and W. Braun, J. Photochem. 8, 341 (1978).

40. Aa. S. Sudbo, P. A. Shulz, E. R. Grant, Y. R. Sher and Y. T. Lee, J. Chem. Phys. 70, 912 (1979).

41. D. S. King and J. C. Stephenson, Chem. Phys. Lett. 51, 48 (1977).

42. J. C. Stephenson, S. E. Bialkowski and D. S. King, J. Chem. Phys. 72, 1161 (1980).

43. G. Hancock, R. J. Hennessy and T. Villis, J. Photochem. 10, 305 (1979).

44. R. V. Ambartzumian, Yu. A. Gorokhov, V. S. Letokhov, G. N. Makarov and A. A. Puretzky, Pis'ma Zh. Eksp. Teor. Fiz. (Russian) 28, 26 (1976). 
45. A. K. Petrov, Yu. N. Samsonov, A. V. Baklanov, V. V. Vizhin and A. M. Orshin, Izvestiya ANSSSR, Chemistry (Russian) 9, 2148 (1976).

46. G. J. Diebold, F. Engelke, D. M. Lubman, J. C. Whitehead and R. N. Zare, J. Chem. Phys. 67, 5407 (1977).

47. N. Rothschild, W. Tsay and D. O. Ham, Optics Commun. 24, 237 (1978).

48. E. R. Grant, M. J. Coggiola, Y. T. Lee, P. A. Schulz, Aa. S. Sudbo and Y. R. Shen, Chem. Phys. Lett. 52, 595 (1977).

49. Aa. S. Sudbo, P. A. Schulz, E. R. Grant, Y. R. Shen and Y. T. Lee, J. Chem. Phys. 68, 1306 (1978).

50. Aa. S. Sudbo, P. A. Schulz, Y. R. Shen and Y. T. Lee, J. Chem. Phys. 69, 2312 (1978).

51. M. H. Yu. M. R. Levy and C. Wittig, J. Chem. Phys. 72, 3789 (1980).

52. C. R. Quick, Jr., J. J. Tiee, T. A. Fischer and C. Wittig, Chem. Phys. Lett. 62, 435 (1979).

53. R. L. Woodin, D. S. Bomse and J. L. Beauchamp, J. Am. Chem. Soc. 100, 3248 (1978).

54. R. L. Woodin, D. S. Bomse and J. L. Beauchamp, Chem. Phys. Lett. 63, 630 (1979).

55. J. C. Stephenson and D. S. King, J. Chem. Phys. 69, 1485 (1978).

56. C. R. Quick and C. Wittig, J. Chem. Phys. 72, 1694 (1980).

57. A. Nitzan and J. Jortner, Chem. Phys. Lett. 60, 1 (1978); J. Chem. Phys. 71, 3524 (1979).

58. A. A. Makarov, G. N. Makarov, A. A. Puretzky and V. V. Tyakht, Appl. Phys. 23, 391 (1980).

59. Y. Haas and G. Yahav, Chem. Phys. Lett. 48, 63 (1977); Chem. Phys. Lett. 35, 41 (1978).

60. M. H. Yu., H. Reisler, M. Mangir and C. Wittig, Chem. Phys. Lett. 62, 439 (1979).

61. M. R. Levy, H. Reisler, M. S. Mangir and C. Wittig, Optical Engineering 19, N1, 029 (1980).

62. T. A. Watson, M. Mangir, C. Wittig and M. R. Levy, J. Phys. Chem. 75, 5311 (1981).

63. A. A. Puretzky and H. Schröder, in: European Conference on Atomic Physics, April 6-10, 1981. Book of Abstracts, Vol. 5A, part I (Ruprecht-Karls-Universität, Heidelberg) p. 288.

64. I. Burak and J. Y. Tsao, Chem. Phys. Lett. 77, 536 (1981).

65. J. Y. Tsao, N. Bloembergen and I. Burak, J. Chem. Phys. 75, 1 (1981).

66. J. G. Calvert and J. N. Pitts, Jr., Photochemistry (Wiley, New York, 1966).

67. A. Yogev and R. M. J. Loewenstein-Benmair, J. Am. Chem. Soc. 95, 8487 (1973).

68. R. V. Ambartzumian, N. V. Chekalin, V. S. Doljikov, V. S. Letokhov and V. N. Lokhman, Optics Commun. 18, 400 (1976); J. Photochem., 6, 55 (1977).

69. C. Kleinermans and H. Wagner, Ber. Bunsenges. Phys. Chem. 81, 1283 (1977).

70. I. Glatt and A. Yogev, J. Am. Chem. Soc. 98, 7087 (1976).

71. A. Yogev and R. M. J. Benmair, Chem. Phys. Lett. 46, 290 (1977).

72. A. Yogev and R. M. J. Benmair, Chem. Phys. Lett. 63, 558 (1979).

73. R. Ansubel and M. H. J. Wijnen, J. Zs. Phys. Chem. 100, 175 (1976).

74. I. N. Knyazev, Yu. A. Kudryavtzev, N. P. Kuzmina and V. S. Letokhov, Zh. Eksp. Teor. Fiz. (Russian) 74, 2017 (1978).

75. S. A. Akhmanov, V. M. Gordietz, V. V. Lazarev, A. V. Mikhoenko and V. Ya. Panchenko, JETPh (Russian) 78, 2171 (1980).

76. J. Troe, J. Chem. Phys. 66, 4745; 4758 (1977).

77. A. C. Baldwin and H. van den Bergh, J. Chem. Phys. 74, 1012 (1981).

78. V. S. Doljikov, Yu. R. Kolomiisky and E. A. Ryabov, Chem. Phys. Lett. 80, 433 (1981).

79. R. Duperrex and H. van den Bergh, Chem. Phys. 40, 275 (1979).

80. J. Stone, E. Thiele, M. F. Goodman, J. C. Stephenson and D. S. King, J. Chem. Phys. 73, 2259 (1980). 
81. A. V. Elezkii, V. D. Udolova and T. A. Udalova, Zh. Eksp. Teor. Fiz. (Russian) 80, 558 (1981).

82. V. N. Bagratashvili, Proceedings of II International School on Laser Applications in Atomic, Molecular and Nuclear Physics, Vilnus, Juli 1981 ("Nauka" Publ. House, Moscow, 1983).

83. T. F. Deutsch, J. Chem. Phys. 70, 1187 (1979).

84. W. Fuss and T. P. Cotter, Appl. Phys. 12, 265 (1977).

85. S. E. Bialkovski and W. A. Guillory, J. Chem. Phys. 67, 2061 (1977).

86. P. Avouris, T. Y. Chan and M. M. T. Loy, J. Chem. Phys. 72, 3522 (1980).

87. R. V. Ambartzumian, Yu. A. Gorokhov, V. S. Letokhov, G. N. Makarov, E. A. Ryabov and N. V. Chekalin, Kvantovaya Electron. (Russian) 2, 2197 (1975).

88. R. V. Ambartzumian, Yu. A. Gorokhov, V. S. Letokhov and G. N. Makarov, Fis'ma Zh. Eksp. Teor. Fiz. (Russian) 21, 375 (1975).

89. D. Tal, U. P. Oppenheim, G. Koren and M. Okon, Chem. Phys. Lett. 48, 67 (1977).

90. V. Yu. Baranov, E. P. Velikhov, S. A. Kazakov, Yu. R. Kolomiisky, V. S. Letokhov, V. D. Pismenny, E. A. Ryabov and A. I. Starodubtzev, Kvantovaya Electron. (Russian) 6, 823 (1979).

91. V. N. Bagratashvili, V. S. Doljikov, V. S. Letokhov and E. A. Ryabov, Pis'ma Zh. Techn. Fiz. (Russian) 4, 1181 (1978).

92. G. I. Abdushelishvili, O. N. Avatkov, V. N. Bagratashvili, V. M. Vezko, V. S. Doljikov, G. G. Esadze, V. S. Letokhov, E. A. Ryabov and G. I. Tkeshelashvili, Pis'ma Zh. Techn. Fiz. (Russian) 5, 849 (1979).

93. S. Bittenson and P. L. Houston, J. Chem. Phys. 67, 4819 (1977).

94. N. C. Peterson, R. G. Manning and W. Braun, J. Res. Nat. Bur. Stand. 83, 117 (1978).

95. K. M. Leary, J. L. Lyman, L. B. Asprey and S. M. Freund, J. Chem. Phys. 68, 1671 (1978).

96. V. N. Bagratashvili, Yu. G. Vainer, V. C. Doljikov, S. F. Koljakov, A. A. Makarov, L. P. Malyavkin, E. A. Ryabov, E. G. Silkis and V. D. Titov, Pis'ma Zh. Eksp. Teor. Fiz. (Russian) 30, 502 (1979) [Sov. Phys. JETP Lett. 30, 471 (1979)].

97. M. J. Coggiola, P. A. Schulz, Y. T. Lee and Y. R. Shen, Phys. Rev. Lett. 38, 17 (1977).

98. J. L. Lyman and K. M. Leary, J. Chem. Phys. 69, 1858 (1978).

99. V. N. Bagratashvili, Yu. R. Kolomiisky, E. A. Ryabov and A. I. Starodubtsev, Kvantovaya Electron. (Russian) 7, 1100 (1980).

100. S. E. Bialkowski and W. A. Guillory, J. Chem. Phys. 68, 3339 (1978).

101. M. L. Lesiecki and W. A. Guillory, J. Chem. Phys. 66, 4239 (1977).

102. J. D. Campbell, M. H. Yu., M. Mangir and C. Wittig, J. Chem. Phys. 69, 3854 (1978).

103. I. P. Herman and J. B. Marling, Chem. Phys. Lett. 64, 75 (1979).

104. D. Proch and H. Schröder, Chem. Phys. Lett. 61, 426 (1979).

105. R. V. Ambartzumian, V. S. Doljikov, V. S. Letokhov, E. A. Ryabov and N. V. Chekalin, Zh. Eksp. Teor. Fiz. 69, 72 (1975).

106. J. L. Lyman and S. D. Rockwood, J. Appl. Phys. 47, 595 (1976).

107. Yu. R. Kolomiisky and E. A. Ryabov, Kvantovaya Electron. (Russian) 5, 651 (1978).

108. V. N. Bourimov, V. S. Letokhov and E. A. Ryabov, J. Photochem. 5, 49 (1976).

109. G. Koren, M. Okon and U. P. Oppenheim, Opt. Commun. 22, 351 (1977).

110. D. K. Evans, R. D. McAlpine and F. K. McClusky, Chem. Phys. Lett. 65, 226 (1979).

111. G. Koren and U. P. Oppenheim, Opt. Commun. 26, 449 (1978).

112. A. Hartford, Chem. Phys. Lett. 57, 352 (1978).

113. M. C. Gower and K. W. Billman, Appl. Phys. Lett. 30, 514 (1977).

114. R. V. Ambartzumian, G. N. Makarov and A. A. Puretzkii, Optics Lett. 3, 103 (1978).

115. R. V. Ambartzumian, Yu. A. Gorokhov, G. N. Makarov, A. A. Puretzkii and N. P. Furzikov, Kvantovaya Electron. (Russian) 4, 1590 (1977). 
116. R. V. Ambartzumian, N. P. Furzikov, Yu. A. Gorokhov, V. S. Letokhov, G. N. Makarov and A. A. Puretzkii, Opt. Lett. 1, 22 (1977).

117. D. F. Heller and G. A. West, Chem. Phys. Lett. 69, 419 (1980).

118. M. J. Rossi, J. R. Barker and D. M. Golden, J. Chem. Phys. 71, 3722 (1979).

119. E. Würzberg, L. J. Kovalenko and P. L. Houston, Chem. Phys. 35, 317 (1978).

120. M. Gauthier, P. A. Hackett, M. Drouin, R. Pilon and C. Willis, Chem. Phys. Lett. 60, 16 (1978).

121. A. B. Horwitz, J. M. Preses, R. E. Weston, Jr. and G. W. Flynn, J. Chem. Phys. 74, 5008 (1981).

122. I. P. Herman and J. B. Marling, J. Chem. Phys. 72, 516 (1980).

123. R. S. Marrison and E. R. Grant, J. Chem. Phys. 71, 3537 (1979).

124. R. V. Ambartzumian, A. P. Dyad'kin, N. P. Fursikov, A. Z. Grasyuk and B. I. Vasilyev, Appl. Phys. 15, 27 (1978).

125. D. K. Evans, R. D. McAlpine and F. K. McClusky, Chem. Phys. 32, 81 (1978).

126. P. Lavigne, J. L. Lachambre and G. Otis, Optics Commun. 22, 75 (1977).

127. S. V. Filseth, J. Danon, D. Feldman, J. D. Campbell and K. H. Welge, Chem. Phys. Lett. 63, 615 (1979).

128. R. D. McAlpine, D. K. Evans and F. K. McClusky, Chem. Phys. 39, 263 (1979).

129. N. V. Chekalin, V. S. Letokhov, V. N. Lokhman and A. N. Shibanov, Chem. Phys. 36, 415 (1979).

130. M. Lussier and J. I. Steinfeld, Chem. Phys. Lett. 50, 175 (1977).

131. C. Reiser, F. M. Lussier, C. C. Jensen and J. I. Steinfeld, J. Am. Chem. Soc. 101, 350 (1979).

132. Z. Karny and R. N. Zare, Chem. Phys. 23, 321 (1977).

133. K. Nagai, M. Katayama, H. Mikuni and M. Takahashi, Chem. Phys. Lett. 62, 499 (1979).

134. J. C. Stephenson, S. E. Bialkowski, D. S. King, E. Thiele, J. Stone and M. F. Goodman, J. Chem. Phys. 74, 3905 (1981).

135. H. Reisler, F. Kong, M. M. Renlund and C. Wittig, J. Chem. Phys. 76, 997 (1982).

136. P. Kolodner, C. Winterfeld and E. Yablonovitch, Optics Commun. 20, 119 (1977).

137. F. Burner and D. Proch, J. Chem. Phys. 68, 4936 (1978).

138. M. C. Gower and K. W. Billman, Optics Commun. 20, 123 (1977).

139. G. Hancock, J. D. Campbell and K. H. Welge, Optics Commun. 16, 177 (1976).

140. S. T. Lin, S. M. Lee and A. M. Ronn, Chem. Phys. Lett. 53, 260 (1978).

141. R. Duperrex and H. van den Bergh, J. Chem. Phys. 70, 5672 (1979).

142. R. R. Karl and J. L. Lyman, J. Chem. Phys. 69, 1196 (1978).

143. J. J. Tiee and C. Wittig, Appl. Phys. Lett. 32, 236 (1978); J. Chem. Phys. 69, 4756 (1978).

144. S. M. Freund and J. L. Lyman, Chem. Phys. Lett. 55, 435 (1978).

145. J. J. Tiee and C. Wittig, Optics Commun. 27, 377 (1978).

146. R. Rabinovitz, A. Kaldor, A. Gnauck, R. L. Woodin and J. S. Gethner, Optics Lett. 7, 212 (1982).

147. J. A. Horsley, P. Rabinovitz, A. Stein, D. M. Cox, R. Brickman and A. Kaldor, IEEE J. Quant. Electr. QE-16, 412 (1980).

148. Ph. Avouris, I. Y. Chan and M. M. T. Loy, J. Chem. Phys. 70, 5315 (1979).

149. A. Hartford, Chem. Phys. Lett. 53, 503 (1978).

150. T. H. Richardson and D. W. Setser, J. Phys. Chem. 81, 2301 (1977).

151. H. L. Dai, A. H. Kung and C. B. Moore, Phys. Rev. Lett. 43, 761 (1979).

152. A. V. Baklanov, Yu. N. Molin and A. K. Petrov, Chem. Phys. Lett. 68, 329 (1979).

153. G. A. West, R. E. Weston and G. W. Flynn, Chem. Phys. 35, 275 (1978).

154. G. A. Fisk, Chem. Phys. Lett. 60, 11 (1978). 
155. C. N. Plum and P. L. Houston, Chem. Phys. 45, 159 (1980).

156. R. J. Jensen, J. K. Hayes, C. L. Cluff and J. M. Thorne, IEEE J. Quant. Electr. QE-16, 1352 (1980).

157. J. L. Lyman, W. C. Danen, A. C. Nilsson and A. V. Nowak, J. Chem. Phys. 71, 1206 (1979).

158. J. D. Campbell, M. H. Yu. and C. Wittig, Appl. Phys. Lett. 32, 413 (1978).

159. A. Hartford and S. A. Tuccio, Chem. Phys. Lett. 60, 431 (1979).

160. V. V. Vizhin, Yu. N. Molin, A. K. Petrov and A. R. Sorokin, Appl. Phys. 17, 385 (1978).

161. P. A. Hackett, M. Gauthier and C. Willis, J. Chem. Phys. 69, 2924 (1978).

162. O. N. Avatkov, E. B. Aslanidi, A. B. Bakhtadze, R. I. Zainullin and Yu. S. Turishchev, Kvantovaya Electron. (Russian) 6, 388 (1979).

163. D. Gutman, W. Braun and W. Tsang, J. Chem. Phys. 67, 4291 (1977).

164. M. J. Rossi, J. R. Barker and D. M. Golden, J. Chem. Phys. 76, 406 (1982).

165. V. V. Vizin, A. K. Petrov and Yu. N. Molin, Dokl. Acad. Nauk (Russian) 243, 129 (1978).

166. X. Zheng, Z. Xu and F. Kong, Chem. Phys. Lett. 85, 418 (1982).

167. S. S. Miller, D. D. DeFord, T. J. Marks and E. Weitz, J. Am. Chem. Soc. 101, 1036 (1979).

168. J. A. Horsley, D. M. Cox, R. B. Hall, A. Kaldor, E. T. Maas, Jr., E. B. Priestley and G. M. Kramer, J. Chem. Phys. 73, 3660 (1980).

169. B. I. Vasil'ev, N. A. Vishyakov, V. T. Galochkin, A. Z. Grasyuk, A. P. Dyadkin, A. K. Zigalkin, V. A. Kovalevsky, V. N. Kosinov, A. N. Oraevsky, A. N. Sukhanov and N. F. Starodubtsev, Pis'ma Zh. Eksp. Teor. Fiz. (Russian) 30, 29 (1979).

170. S. F. Filseth, J. Danon, D. Feldman, J. D. Campbell and K. H. Welge, Chem. Phys. Lett. 66, 329 (1979).

171. Yu. N. Samsonov and A. K. Petrov, Chem. Phys. Lett. 84, 183 (1981).

172. A. J. Colussi, S. W. Benson, R. J. Hwang and J. J. Tiee, Chem. Phys. Lett. 52, 349 (1977).

173. J. B. Marling and I. P. Herman, Appl. Phys. Lett. 34, 439 (1979).

174. J. C. Stephenson, D. S. King, M. F. Goodman and J. Stone, J. Chem. Phys. 70, 4496 (1979).

175. R. V. Ambartzumian, Yu. A. Gorokhov, V. S. Letokhov, G. N. Makarov and A. A. Puretzky, Phys. Lett. 56A, 183 (1976). 


\section{ISOTOPIC SELECTIVITY OF MULTIPLE PHOTON DISSOCIATION OF MOLECULES}

This chapter is concerned with one of the most important and beautiful properties of multiple photon dissociation of molecules, that is, its selectivity. As applied to the process of MP dissociation, the concept of selectivity can be considered in two aspects. One can speak of the selective excitation of a specific vibrational mode (or modes) and the breaking of the bond corresponding to that mode. The realization of such a type of selectivity was discussed in the foregoing chapters as well as in Chapter 9. The data available shows that the prospective in this case is rather limited. The second type of selectivity is selective excitation and dissociation of a specified sort of molecules in their mixture including the mixture of different isotopes. In the latter case irradiation of isotopic mixtures of molecules produced the most striking results which open up wide possibilities for different applications of the process of MP dissociation. It is just this property of isotopic selectivity that is the subject of the present chapter.

The very first experiments ${ }^{1,2}$ demonstrated the possibility of molecular dissociation by IR radiation for a time shorter than the characteristic times of all known collisional processes. This raised the hope for providing the selectivity of MP dissociation. However at that time it was not evident at all that the excitation was "soft" enough to obtain selectivity under such collisionless conditions. It was not clear whether the difference of the frequencies of the vibrational transition under excitation, say, within the limits of several dozens of $\mathrm{cm}^{-1}$, is sufficient for selective dissociation of specified molecules. The positive answer to this question was given by experiments with molecules containing different isotopes.

The isotopic selectivity of MP dissociation was first shown in experiments with the ${ }^{10} \mathrm{BCl}_{3}$ and ${ }^{11} \mathrm{BCl}_{3}$ molecules. ${ }^{3}$ In this case the isotope shift in the frequency of the vibration being excited was $39 \mathrm{~cm}^{-1}$. A great number of subsequent experiments with different molecules showed that the case of the $\mathrm{BCl}_{3}$ molecule was not unique and that the selectivity of MP dissociation was its characteristic property and resulted from the very mechanism of vibrational excitation of molecules by laser radiation. In the experiments on separation of the uranium isotopes ${ }^{235} \mathrm{U}$ and ${ }^{238} \mathrm{U}^{4}$ isotopic selectivity of dissociation was observed when the isotope shift in the excited state was just $0.7 \mathrm{~cm}^{-1}$. 
Laser isotope separation is one of the most important potential applications of the selective molecular MP dissociation. Therefore, the great majority of experiments dealing with the study of the kinetics of enrichment, the dependence of selectivity on the conditions of excitation, have been carried out with different isotopic molecules but the results obtained hold true for mixtures of any other species.

Multiple photon excitation and dissociation of a two-component mixture, for example a mixture of two isotopes, have a number of very essential specific features as compared to the case of a onecomponent medium. The study of the isotopic selectivity of MP dissociation has revealed the means of obtaining maximum selectivity of MP excitation and dissociation. These studies make it possible to gain some insight into the very process of multiphoton excitation of molecules including subsequent evolution of highly excited molecules. A brilliant example of this is the very discovery of the effect of isotopic selectivity of MP dissociation that was the final and convincing proof for the possibility of collisionless excitation and dissociation of molecules by IR radiation.

Many experiments on isotopic selectivity of MP molecular dissociation have thus been far performed. These experiments have covered many isotopes, from light ones (hydrogen, deuterium) to heavy ones (osmium, uranium) contained in very different molecules. Table 8.1 presents the basic results. It gives the isotopes and compounds used in the experiments as well as the values obtained for the parameters of selectivity.

The results of studies carried out for the isotopic selectivity of molecular MP dissociation are discussed below in more detail. Special consideration is given to the factors which determine the degree of selectivity of this process and to the possibilities of its increase. We are going to begin with defining the parameters describing the selectivity and their experimental measurement.

\subsection{Characteristics of isotopically selective MP dissociation}

\subsubsection{Kinetics of enrichment of a two-component mixture}

When a mixture of $A B$ type molecules with different $A$ isotopes is irradiated, there will be preferential dissociation of molecules of that isotopic composition of the absorption band to which the laser radi- 
TABLE 8.1

Isotope separation by multiplephoton dissociation of molecules

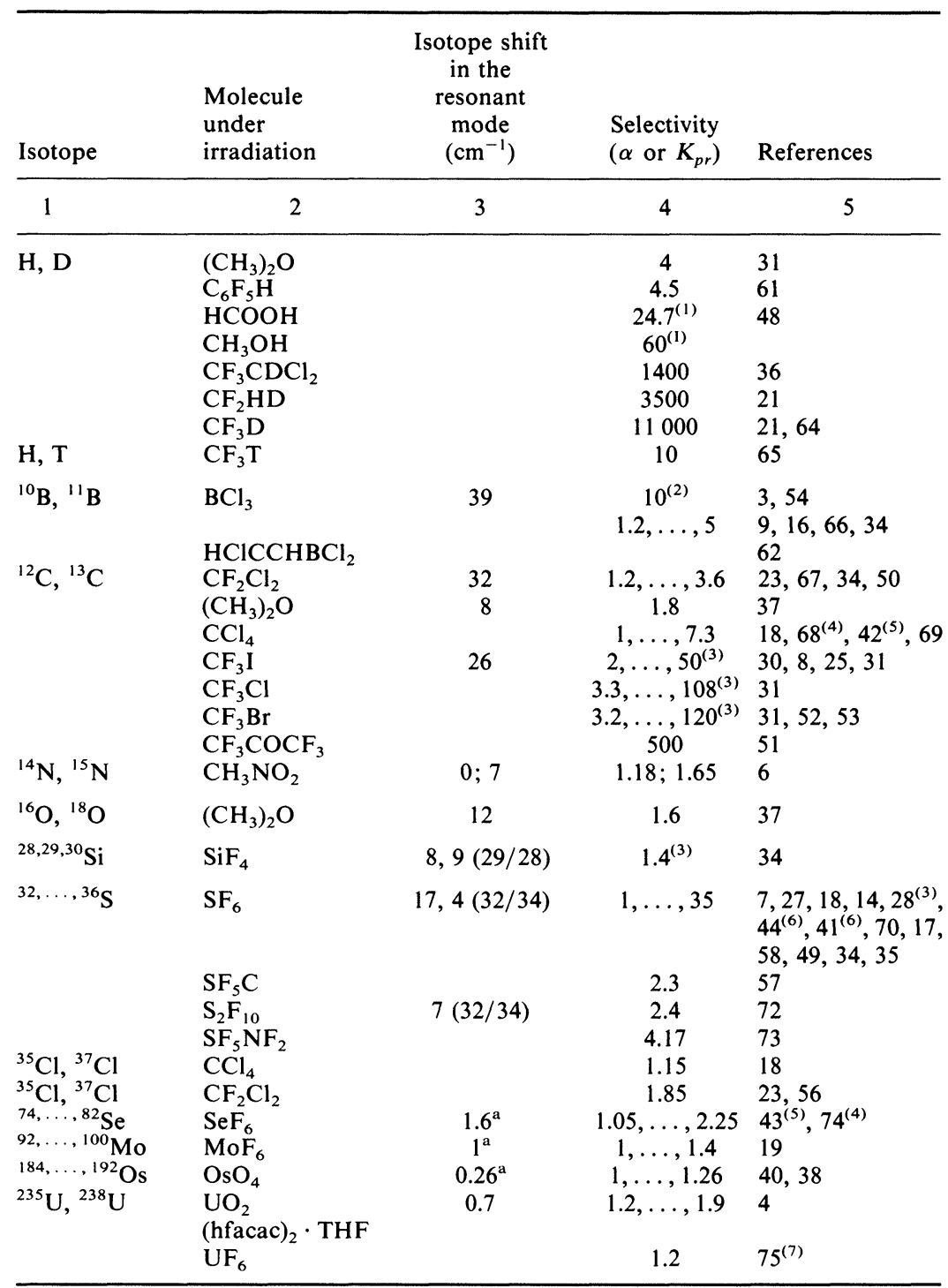

a) Isotopic shift per atomic mass.

(1) Dissociation by HF laser radiation. (2) Measurement in intermediate reaction products. (3) Cooling. (4) Dissociation by $\mathrm{NH}_{3}$ laser radiation. (5) Two-frequency dissociation by $\mathrm{NH}_{3}$ and $\mathrm{CO}_{2}$ lasers. (6) Two-frequency dissociation by $\mathrm{CO}_{2}$ laser. (7) Two-frequency dissociation by $16 \mu$ laser. 
ation frequency is tuned. For simplicity, we shall consider a twocomponent mixture containing ${ }^{i} A$ and ${ }^{k} A$ isotopes. Let the laser radiation perform preferential dissociation of ${ }^{i} A B$ molecules. It is quite obvious that, as the gas is exposed to radiation, the relative concentration of ${ }^{i} A B$ molecules in the volume will decrease and that of ${ }^{k} A B$ will increase. Thus the residual gas is enriched with the ${ }^{k} A$ isotope. The value of this enrichment increases with exposure time, and it may be quite high with a rather large expenditure of the gas under irradiation.

In the dissociation products, on the contrary, enrichment with the excited ${ }^{i} A$ isotope takes place. The value of enrichment in this case depends on the degree of dissociation selectivity of ${ }^{i} A B$ molecules relative to ${ }^{k} A B$ and drops with irradiation because the ${ }^{k} A B$ molecules dissociate, too, even though with a lower rate than ${ }^{i} A B$. Thus the value of enrichment of the dissociation products with the ${ }^{i} A$ isotope at large dissociation of the gas exposed will be small.

We shall now, to obtain quantitative relations, consider the kinetics of enrichment in more detail. Let the dissociation yield per pulse in the volume under irradiation be $\beta_{i}$ for ${ }^{i} A B$ and $\beta_{k}$ for ${ }^{k} A B$ molecules. Since there is preferential dissociation of ${ }^{i} A B, \beta_{i}>\beta_{k}$. We can characterize the selectivity of MP dissociation by the parameter

$$
\alpha=\frac{\beta_{i}}{\beta_{k}}
$$

In other words, $\alpha$ is the ratio of probabilities of dissociation in one pulse for the two species ${ }^{i} A B$ and ${ }^{k} A B$ in the volume irradiated. Such a definition of selectivity is similar to that used in the conventional methods of isotope separation. In laser isotope separation $\alpha$ also serves as a measure of selectivity for the elementary reaction.

The concentrations of ${ }^{i} A B$ and ${ }^{k} A B$ molecules in the cell $N_{i}$ and $N_{k}$ respectively can be written as

$$
N_{i}=N_{i}^{0}\left(1-\beta_{i} \Gamma\right)^{2} \quad N_{k}=N_{k}^{0}\left(1-\beta_{k} \Gamma\right)^{n}
$$

where $N_{i}^{0}, N_{k}^{0}$ denote the initial concentrations, $n$ is the number of radiation pulses, $\Gamma$ is the ratio of the exposed gas volume to the total volume of the cell. Expressions (8.2) are valid when $\beta_{i}$ and $\beta_{k}$ are independent of gas pressure. This takes place when the dissociation of ${ }^{i} A B$ and ${ }^{k} A B$ molecules results from their purely radiative excitation, particularly at low gas pressures (in more detail see Section 8.5). 
In the process of preferential dissociation of ${ }^{i} A B$ molecules the residual gas is enriched with the ${ }^{k} A$ isotope. The value of enrichment is characterized by the enrichment factor in residual gas $K_{\text {res }}$

$$
K_{\mathrm{res}}(k / i)=\frac{\left[{ }^{k} A B\right]_{n}}{\left[{ }^{i} A B\right]_{n}}: \frac{\left[{ }^{k} A B\right]_{0}}{\left[{ }^{i} A B\right]_{0}}
$$

where the index 0 relates to the unirradiated mixture and $n$ to the irradiated. Using (8.3) we can obtain

$$
K_{\mathrm{res}}(k / i)=\frac{\left(1-\beta_{k} \Gamma\right)^{n}}{\left(1-\beta_{i} \Gamma\right)^{n}}=\frac{\left(1-\beta_{i} \Gamma / \alpha\right)^{n}}{\left(1-\beta_{i} \Gamma\right)^{n}}
$$

In the dissociation products enrichment with the ${ }^{i} A$ isotope occurs and the enrichment factor in the dissociation products $K_{\mathrm{pr}}$ will be

$$
K_{\mathrm{pr}}(i / k)=\frac{\left[{ }^{i} A\right]_{\mathrm{pr}}}{\left[{ }^{k} A\right]_{\mathrm{pr}}}: \frac{\left[{ }^{i} A\right]_{0}}{\left[{ }^{k} A\right]_{0}}=\frac{\Delta\left[{ }^{i} A B\right]}{\Delta\left[{ }^{k} A B\right]}: \frac{\left[{ }^{i} A B\right]_{0}}{\left[{ }^{k} A B\right]_{0}}=\frac{1-\left(1-\beta_{i} \Gamma\right)^{n}}{1-\left(1-\beta_{k} \Gamma\right)^{n}}
$$

With the use of (8.4) and (8.5) we can get the dependence of the enrichment factor in the residual gas and in the dissociation products on the number of pulses as a portion of gas is irradiated in the cell (see Figure $8.1^{5}$ ). Accordingly one can see that in the residual gas the value of $K_{\text {res }}$ increases with irradiation and, with sufficient exposure, it is possible to get any desired value of $K_{\text {res. }}$. In the dissociation products the enrichment factor drops with irradiation. With a small

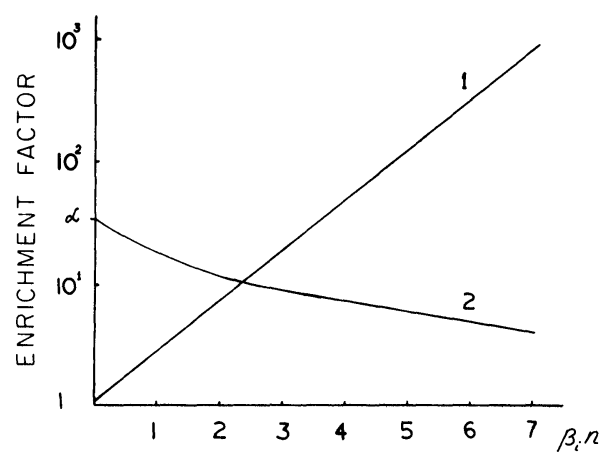

FIGURE 8.1 Dependence of the enrichment factor $K$ on irradiation time (in units of $\beta_{i} n$ ) in exciting the ${ }^{i} A$ isotope. Curve 1 denotes the enrichment in the residual gas, curve 2 in the dissociation products. ${ }^{5}$ 
expenditure of gas in the cell, i.e., when $\beta_{i} \Gamma n<1$, the value of $K_{\mathrm{pr}}$ reaches its maximum equal to the selectivity $K_{\mathrm{pr}}=\beta_{i} / \beta_{k}=\alpha$.

\subsubsection{Experimental methods for the measurement of selectivity parameters}

$K_{\mathrm{pr}}$ and $K_{\text {res }}$ are values directly measured in most experiments. By using the values obtained for $K_{\mathrm{pr}}$ and $K_{\text {res }}$ one can find the value of $\alpha$. The determination of $\alpha$ is analyzed comprehensively in. ${ }^{6}$

Figure 8.2 schematically shows the process of isotopically selective molecular dissociation and illustrates the available possibilities of the control of selectivity. In most of the experiments the measurements were taken by analyzing the final results of irradiation. In these cases, from (8.4) and (8.5), one needs to know the final content of the isotopes in the gas under irradiation and the dissociation products to evaluate $K_{\mathrm{pr}}$ and $K_{\text {res }}$. The simplest way to do this is by IR absorption spectroscopy. This method was first used ${ }^{7}$ for isotopically selective dissociation of $\mathrm{SF}_{6}$ but it is not always convenient to apply particularly when the isotope concentrations differ greatly.

A more universal method to determine $K_{\mathrm{pr}}$ and $K_{\text {res }}$ is measuring with the use of a mass spectrometer which provides a required measurement accuracy over a wide concentration range. It should be noted that, from (8.4) and (8.5), the simultaneous mass-spectroscopic measurement of $K_{\mathrm{pr}}$ and $K_{\text {res }}$ allows measuring the values of $\beta_{i}$ and $\beta_{k}$ as well. This seems to be the only method at present that enables the value of $\beta_{i}$ to be measured when the concentration of the ${ }^{i} A$ isotope $x_{0 i} \ll 1$.

The $K_{\mathrm{pr}}$ and $K_{\text {res }}$ values, however, are integral characteristics and depend on gas consumption (see Figure 8.1). The selectivity $\alpha$ is of great physical interest. Strictly speaking, it is the enrichment factor in products under single-shot irradiation. In practice it is not always possible to measure $K_{\mathrm{pr}}$ with $n=1$, especially with $\Gamma \ll 1$, because of the limited sensitivity of the measuring equipment. However at a low gas consumption, as shown above, i.e., with $\beta \Gamma n \ll 1, K_{\mathrm{pr}}=\alpha$. If the condition of a small consumption is not fulfilled, the value of $\alpha$ can be found, in principle, from relations (8.4) and (8.5).

The value of selectivity obtained in the primary dissociation products has been so far assumed not to decrease in subsequent chemical reactions to yield stable end products but this is not always so. In the 


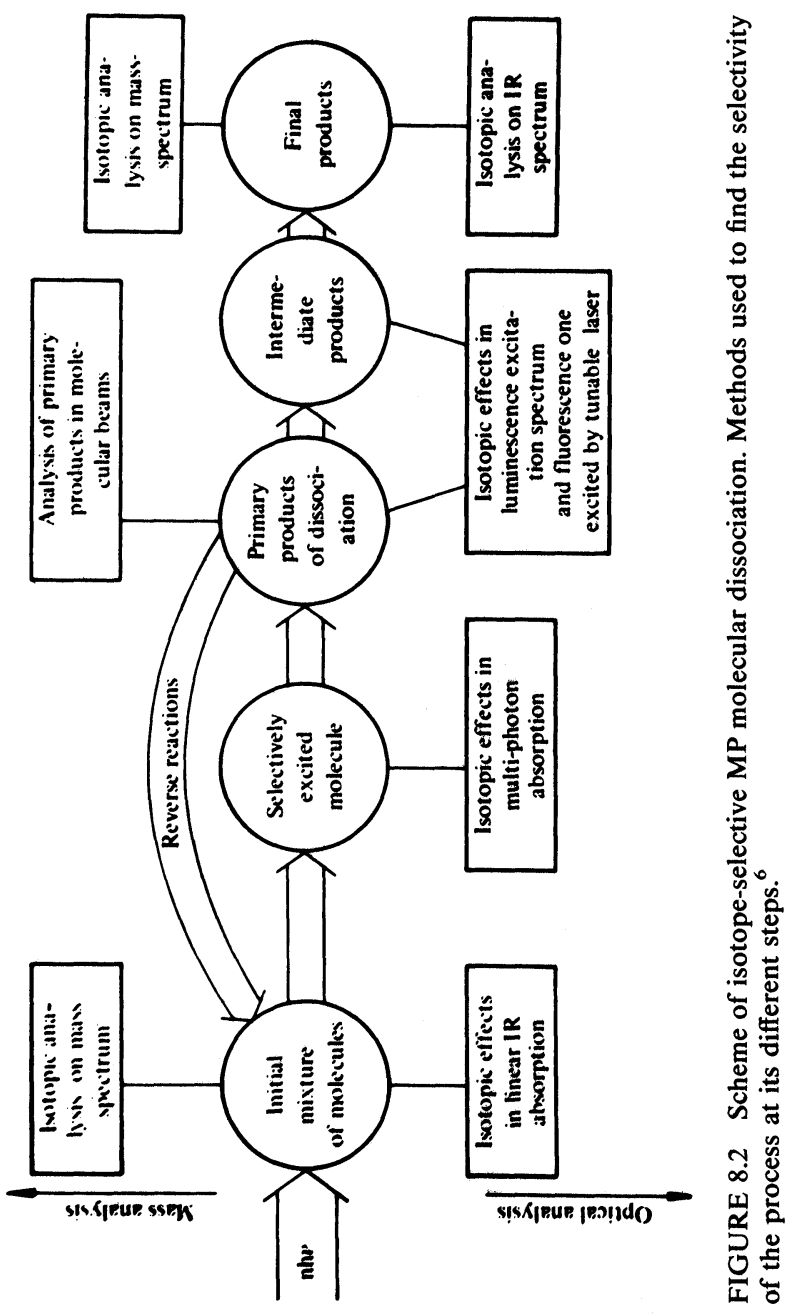


experiments with $\mathrm{BCl}_{3}{ }^{9}$ it was found that the enrichment factor in the products depended on the types of scavengers used to bind the primary dissociation fragments. This point is discussed below in more detail. We want to note that in this case the actual primary selectivity can be determined in several ways (see Figure 8.2). First, molecular beams can be applied. ${ }^{10,11}$ In this case it is quite possible to measure enrichment immediately in primary dissociation products.

The second possibility of measuring the primary value of $\alpha$ is related to studying isotope effects in the spectrum of primary dissociation fragments or intermediate products of subsequent chemical reactions. We should emphasize here the possibility of analysis of the fluorescence spectra of radicals when they are excited with tunable dye lasers. ${ }^{12,13}$ Furthermore, it is possible to study isotope effects in the chemiluminescence spectra of products at the primary stages of chemical reactions. An example ${ }^{3}$ of the latter method was the determination of the selectivity of MP dissociation from analyzing the emission spectra of the electronically excited radicals ${ }^{10} \mathrm{BO}^{*}$ and ${ }^{11} \mathrm{BO}$ * formed by the dissociation of $\mathrm{BCl}_{3}$ in the presence of oxygen.

\subsection{Resonant properties of selectivity}

Studies of MP dissociation show that selectivity results, first of all, from the resonant character of multiple photon excitation. The resonant properties of dissociation, especially the spectra of MP excitation and dissociation of monoisotopic molecules, were considered in the previous chapters. In this section we are going to draw our attention to the results obtained from irradiating a two-component mixture consisting of molecules with different isotopes. Among other things we shall consider the dependence of selectivity on the exciting radiation frequency as well as the effects taking place in MP absorption.

\subsubsection{Dependence of MP dissociation selectivity on IR laser frequency}

Excitation of fundamental modes. The form of the spectral dependence of dissociation yield obtained for a particular molecule makes it possible to determine the maximum value of selectivity that can be reached in dissociation of molecules with a certain isotope. Indeed, if the dependence of $\beta$ on the exciting radiation frequency $\Omega$ is known for each isotope, it is quite obvious that the ratio of these values at 
any value of $\Omega$ must yield the greatest possible value of $\alpha$. Thus, given the MP dissociation spectrum for each of the isotopes, one can determine the spectral dependence of selectivity on the radiation frequency. This conclusion has been supported in a number of experiments.

In the experiments with $\mathrm{SF}_{6}{ }^{14}$ the enrichment factor of the ${ }^{32} \mathrm{~S}$ and ${ }^{34} \mathrm{~S}$ isotopes in the products $K_{\mathrm{pr}}$ was measured as a function of excitation frequency. The results are presented in Figure 8.3. In the same figure one can see the dependence of the dissociation yield $\beta_{32}$ measured for ${ }^{32} \mathrm{SF}_{6}$ molecules. The curve for $\beta_{34}$ was constructed by transferring the dependence for $\beta$ by the value of isotope shift $\Delta \nu_{\text {is }}$ under the assumption that the form of the spectral dependence of dissociation yield for ${ }^{32} \mathrm{SF}_{6}$ and ${ }^{34} \mathrm{SF}_{6}$ is the same. It may be seen that the value of $K_{\mathrm{pr}}(32 / 34)$ is maximum in the short-wave spectral range where $\beta_{32}>\beta_{34}$ and preferential dissociation of ${ }^{32} \mathrm{SF}_{6}$ takes place. With a decrease in $\Omega$ the value of $K_{\mathrm{pr}}(32 / 34)$ is reduced and there is no selectivity $\left(K_{\mathrm{pr}}=1\right)$ where the curves for $\beta_{32}$ and $\beta_{34}$ intersect. With further decrease in frequency, when $\beta_{34}$ becomes higher than $\beta_{32}$, the products are enriched with the ${ }^{34} \mathrm{~S}$ isotope.

The similar dependence of dissociation selectivity on laser radiation frequency was obtained ${ }^{16}$ in an experiment with $\mathrm{BCl}_{3}$ with the natural abundance of boron isotopes ${ }^{10} \mathrm{~B}:{ }^{11} \mathrm{~B}=19: 81$. The measurements were taken as the laser radiation was focused into a cell with $\mathrm{BCl}_{3}(0.25$ Torr $)$

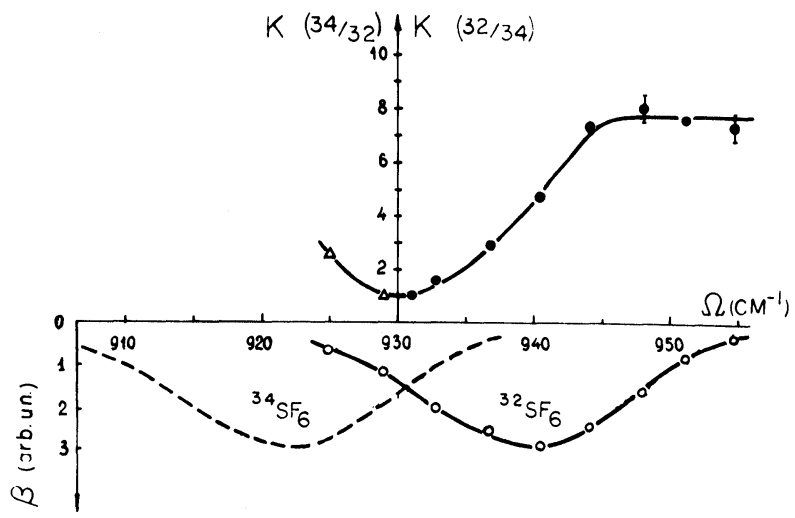

FIGURE 8.3 Spectral dependence of the enrichment factor in the dissociation products of $\mathrm{SF}_{6}$ at a gas pressure of 0.2 Torr. Below are the spectral curves of dissociation yield for ${ }^{32} \mathrm{SF}_{6}$ and ${ }^{34} \mathrm{SF}_{6}{ }^{14}$ 
and air (4 Torr). Oxygen was used to bind the dissociation products of $\mathrm{BCl}_{3}$. The results are given in Figure 8.4. It may be seen that the curve of dissociation yield for ${ }^{11} \mathrm{BCl}_{3}$ is displaced by $17 \mathrm{~cm}^{-1}$ from the maximum of its linear absorption. With the isotope shift in the frequency of excited vibration of ${ }^{10} \mathrm{BCl}_{3}$ and ${ }^{11} \mathrm{BCl}_{3} \Delta \nu_{\mathrm{is}}=39 \mathrm{~cm}^{-1}$ one might expect that $\beta_{11}$ would be equal to $\beta_{10}$, i.e., there would be no selectivity at the frequency $\Omega=956 \mathrm{~cm}^{-1}$. The experimental measurements supported this conclusion. Worthy of notice is the asymmetrical form of the dependence $\alpha=\alpha(\Omega)$-the dissociation selectivity turns out to be higher when the lighter ${ }^{10} \mathrm{BCl}_{3}$ isotope is excited. This is related $^{16}$ to the fact that during a laser pulse the molecules, nevertheless, had time to undergo collisions. As a result of $V-V$ exchange, a fraction of molecules of the unexcited isotope falls within the region with a high density of vibrational states (vibrational quasi-continuum). Now these molecules can absorb laser radiation and dissociate which leads to a decrease in selectivity. The spectral characteristics of the quasi-continuum have such a form that the absorption cross-section in the case of a heavier isotope $\left({ }^{11} \mathrm{BCl}_{3}\right)$ is larger than in the case of

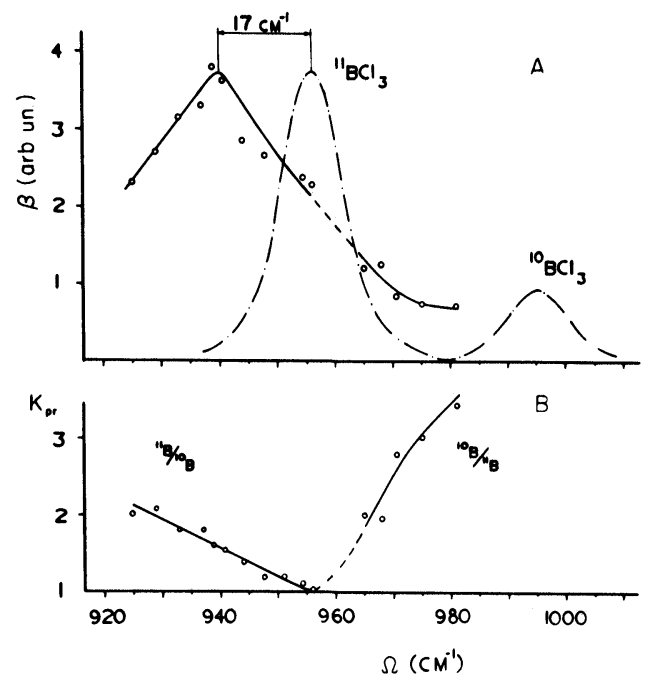

FIGURE 8.4 Dependence of the dissociation yield $\beta$ (a) and the enrichment factor in products $K_{\mathrm{pr}}$ (b) on the laser radiation frequency in the dissociation of $\mathrm{BCl}_{3} .{ }^{16} \mathrm{BCl}_{3}$ pressure is 0.25 Torr, the pressure of the $\mathrm{O}_{2}+\mathrm{N}_{2}$ is 4 Torr. The dashed line denotes the linear absorption spectrum. 
a lighter one $\left({ }^{10} \mathrm{BCl}_{3}\right)$. This results in an asymmetrical form of the dependence $\alpha=\alpha(\Omega)$ (Figure 8.4).

It should be noted that the form of the spectral dependence of dissociation yield may be governed by the energy fluence of exciting radiation $\Phi$. So the form of the spectral dependence of selectivity may be also governed by $\Phi$. It is this circumstance that is related to the experimental fact observed in ${ }^{17} \mathrm{SF}_{6}$. It was found that at the $\mathrm{P}(34)$ line of $\mathrm{CO}_{2}$ laser with $\Phi>6 \mathrm{~J} / \mathrm{cm}^{2}$ preferential dissociation of ${ }^{34} \mathrm{SF}_{6}$ and enrichment with the ${ }^{34} \mathrm{~S}$ isotope in the products took place. With $\Phi<6 \mathrm{~J} / \mathrm{cm}^{2}$, on the contrary, ${ }^{32} \mathrm{SF}_{6}$ mainly dissociated and the products were enriched with ${ }^{32} \mathrm{~S}$.

Excitation of combination bands. The possibility of selective dissociation during excitation of weak combination molecular vibrations essentially widens the area of application of MP dissociation.

In experiments ${ }^{1}$ with $\mathrm{SiF}_{4}$ to explain the dissociation under $\mathrm{CO}_{2}$ laser radiation in the $10.6 \mu$ region it was suggested that its excitation was through combination vibrational transitions. Other experiments with different molecules confirmed such an assumption.

It was possible, ${ }^{18}$ for example, to observe dissociation of $\mathrm{SF}_{6}$ as the $\mathrm{CO}_{2}$ laser radiation was tuned to the combination bands $\nu_{2}+\nu_{6}$ and $\nu_{2}+\nu_{3}-\nu_{5}$. In the second case the residual $\mathrm{SF}_{6}$ was enriched with the ${ }^{34} \mathrm{~S}$ isotope. It is believed ${ }^{18}$ that the value of selectivity during excitation of combination vibrations and overtones may be even higher than during excitation of the fundamental vibration. This can be explained by a smaller value of power broadening in the first case due to a smaller dipole moment of transition.

The isotopic selectivity of dissociation during excitation of combination modes was also demonstrated in experiments with $\mathrm{CCl}_{4} \cdot{ }^{18} \mathrm{In}$ these experiments the molecules were irradiated at different $\mathrm{CO}_{2}$ laser frequencies within the absorption band $\nu_{1}+\nu_{2}+\nu_{4}$. Enrichment of the products with both the ${ }^{13} \mathrm{C}$ and ${ }^{37} \mathrm{Cl}$ isotopes could be observed.

The separation of molybdenum isotopes ${ }^{19}$ has been achieved with $\mathrm{CO}_{2}$ laser irradiation of the combination band $\nu_{3}+\nu_{5}$ of the $\mathrm{MoF}_{6}$ molecule.

Thus, the process of MP molecular dissociation can be isotopically selective acting on weak combination vibrations. Even though during excitation of this vibrations the dissociation yield is smaller than in case of fundamental vibration such a method of excitation can be used in the selective dissociation of molecules. In particular, it can 
be used when the excitation of the fundamental vibrations is impossible due to the absence of a proper laser. In this case one can apply the two-frequency method of dissociation with excitation at the first step of an appropriate combination mode.

\subsubsection{Selectivity of multiple photon absorption}

A characteristic feature of MP dissociation as opposed to other photophysical processes based on the excitation in one step is that not all molecules having absorbed IR laser photons are able to dissociate. Only those molecules can dissociate which have acquired energy $E$ being higher than the dissociation energy, i.e., $E>D$. Therefore, MP molecular dissociation is characterized by a strong dependence of the dissociation yield $\beta$ on the average excitation level of molecules. Especially strong dependence is with $\beta \ll 1$ when a small fraction of molecules dissociates at the tail of the energy distribution. In the case of $\mathrm{CF}_{3} \mathrm{I}$, for example, when the energy absorbed per molecule varies between $\bar{\varepsilon}=3000 \mathrm{~cm}^{-1}$ and $\bar{\varepsilon}=7600 \mathrm{~cm}^{-1}$, the dissociation yield changes by more than two orders, i.e., from $\beta=7 \cdot 10^{-4}$ to $\beta=10^{-1}$. $^{20}$ So even when the values of $\beta$ for each of the isotopes differ greatly, i.e., $\alpha \gg 1$, the difference in the average level of excitation of these molecules may be not so drastic at all. Hence, the parameter $\alpha$ serving as a measure of dissociation selectivity may badly reflect the difference in the excitation level of molecules with different isotopes.

Excitation selectivity. $\alpha_{\mathrm{ex}}$ is a measure of the difference in multiphoton absorption of two isotopic molecules. This value can be introduced in a natural way as a ratio of average excitation levels for two species, i.e.,

$$
\alpha_{\mathrm{ex}}(i / k)=\frac{\overline{\varepsilon_{i}}}{\overline{\varepsilon_{k}}}
$$

Since $\bar{\varepsilon}=\sigma \Phi$, where $\sigma$ is the MP excitation cross-section, it follows from (8.6) that

$$
\alpha_{\mathrm{ex}}=\frac{\sigma_{i}}{\sigma_{k}}
$$

which agrees with the definition of excitation selectivity. ${ }^{21}$

The $\alpha_{\text {ex }}$ value, as well as the $\alpha$ value above, can be determined from the MP excitation spectra for each of the isotopes. In some cases this enables predicting the selectivity in dissociation. On the basis of such 
measurements ${ }^{6}$ isotopic selectivity of MP dissociation of $\mathrm{CH}_{3}{ }^{15} \mathrm{NO}_{2}$ molecules (see Section 8.2.3) was revealed in the absence of an isotope shift in the linear absorption spectrum, and the possibility of selective excitation ${ }^{22}$ of ${ }^{238} \mathrm{UF}_{6}$ molecules in their mixture with ${ }^{235} \mathrm{UF}_{6}$ by the $\mathrm{CF}_{4}$ laser has been demonstrated.

It must be noted that both the value of $\alpha_{\mathrm{ex}}$ and its dependence on the conditions of excitation (pressure, pulse duration, and so on) have not been so far extensively measured. We can point to the results obtained for different deuterium-containing molecules. ${ }^{21}$ According to the above, $\alpha_{\text {ex }}$ turns out to be smaller than $\alpha$. As the $\mathrm{CF}_{3} \mathrm{CDCl}_{2}$ and $\mathrm{CF}_{3} \mathrm{CHCl}_{2}$ molecules were excited under the same experimental conditions, the excitation selectivity was found to be $\alpha_{\mathrm{ex}}=59$ while $\alpha>10^{3}$. Much higher selectivity was reached during excitation of $\mathrm{CF}_{3} \mathrm{D}$. In the presence of $\mathrm{CF}_{3} \mathrm{H}$ the value of $\alpha_{\text {ex }}$ obtained was 4900 with $\Phi=1 \mathrm{~J} / \mathrm{cm}^{2}$ and the pulse duration $\tau_{p}=2 \mathrm{~ns}$. At smaller values of $\Phi$ the value of $\alpha_{\mathrm{ex}}$ was higher. It should be noted that the difference between $\alpha_{\text {ex }}$ and $\alpha$ for this "small" (as compared to $\mathrm{CF}_{3} \mathrm{CCl}_{2} \mathrm{D}$ ) molecule turned out to be much smaller.

In conclusion it must be said that high values of $\alpha_{\mathrm{ex}}$ are essential in a number of applications of isotopically-selective MP dissociation. It is this parameter that in many respects determines the value of energy consumption. Indeed, if there is a mixture of two components " $i$ " and " $k$ " and the content of the excited component " $i$ " is $x_{0} \ll 1$, the fraction of total absorbed energy stored in the required component

$$
\frac{\Delta E_{i}}{\Delta E_{i}+\Delta E_{k}}=\frac{\overline{\varepsilon_{i}} x_{0}}{\overline{\varepsilon_{i}} x_{0}+\left(1-x_{0}\right) \overline{\varepsilon_{k}}}=\frac{1}{1+\left(1-x_{0}\right) / \alpha_{\mathrm{ex}} x_{0}}
$$

From (8.8) it follows that, only when $\alpha_{\mathrm{ex}}=\overline{\varepsilon_{i}} / \overline{\varepsilon_{k}}>1 / x_{0}$, is the main portion of absorbed energy used for the excitation of the desired molecules.

\subsubsection{Selectivity of MP excitation and dissociation for a small isotope shift}

We have discussed thus far the results of experiments in which the isotopic selectivity of dissociation was observed in acting on vibrations (fundamental or combination) with an isotope shift in the linear absorption spectrum but isotopic selectivity can be also attained ${ }^{6}$ in the absence of an isotope shift. 
Experiments have been carried out with $\mathrm{CH}_{3} \mathrm{NO}_{2}$ that has two vibrations $\nu_{7}$ and $\nu_{13}$ in the region of the laser. The frequency shift in the $\nu_{7}$ vibration of $\mathrm{CH}_{3}{ }^{15} \mathrm{NO}_{2}$ and $\mathrm{CH}_{3}{ }^{14} \mathrm{NO}_{2}$ molecules equals $7 \mathrm{~cm}^{-1}$ (see Figure 8.5a). In the linear absorption spectrum of the $\nu_{13}$ band there was no isotope shift observed.

However in acting on the $\nu_{13}$ vibration with intense radiation $\left(10^{7}, \ldots, 10^{9} \mathrm{~W} / \mathrm{cm}^{2}\right)$ the excitation spectrum of $\mathrm{CH}_{3}{ }^{15} \mathrm{NO}_{2}$ and $\mathrm{CH}_{3}{ }^{14} \mathrm{NO}_{2}$ was found to be deformed in different ways (Figure 8.5b). This difference is equivalent to the appearance of an isotope "shift" of about $5 \mathrm{~cm}^{-1}$. The difference in the spectrum of multiple photon absorption brought about a corresponding difference in the spectral dependence of the dissociation yield of these molecules (Figure 8.5c). The curves from Figure $8.5 \mathrm{c}$ were obtained from measuring the luminescence intensity electronically excited dissociation fragments. The maximum difference in the luminescence signal in excitation of two isotopic modifications was $\beta_{15} / \beta_{14}=2$. As noted above, the difference in the values of dissociation yield makes it possible to anticipate obtaining isotopic selectivity of dissociation in exciting the mixture of these molecules.
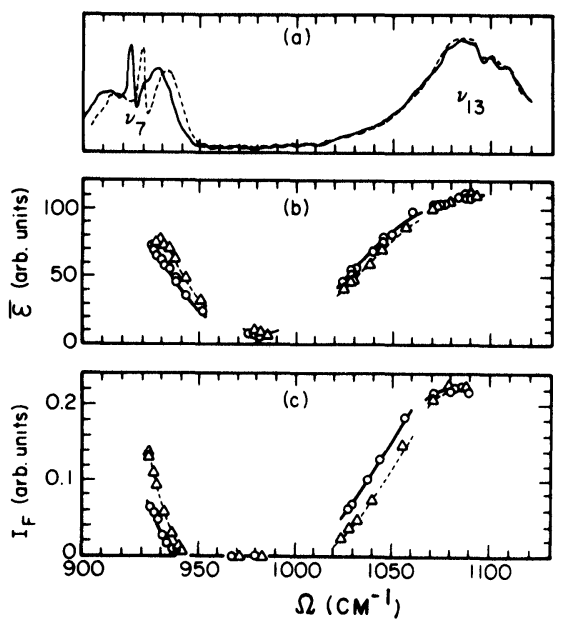

FIGURE 8.5 Isotopic effects for the $\nu_{7}$ and $\nu_{13}$ modes of $\mathrm{CH}_{3}{ }^{14} \mathrm{NO}_{3}$ and $\mathrm{CH}_{3}{ }^{15} \mathrm{NO}_{2}:{ }^{6}$ (a) linear absorption spectrum ; (b) MP absorption spectrum, $I=5 \cdot 10^{9} \mathrm{~W} / \mathrm{cm}^{2} ;$;c) spectral dependence of visible luminescence signal in dissociation of $\mathrm{CH}_{3} \mathrm{NO}_{2}, I=$ $5 \cdot 10^{9} \mathrm{~W} / \mathrm{cm}^{2}$. The dashed line is $\mathrm{CH}_{3}{ }^{14} \mathrm{NO}_{2}$, the solid curves are $\mathrm{CH}_{3}{ }^{15} \mathrm{NO}_{2}$. 
Direct experiments on nitrogen isotope separation were performed with the equimolecular mixture of $\mathrm{CH}_{3}{ }^{15} \mathrm{NO}_{2}$ and $\mathrm{CH}_{3}{ }^{14} \mathrm{NO}_{2}$ irradiated at a frequency of $1025.3 \mathrm{~cm}^{-1}$. The total gas pressure was 0.5 Torr. The measurements show that under these conditions preferential dissociation of $\mathrm{CH}_{3}{ }^{15} \mathrm{NO}_{2}$ takes place and the enrichment factor $K_{\mathrm{pr}}(15 / 14)$ is equal to $1.18 \pm 0.08$. The smaller value of $K_{\mathrm{pr}}$, as compared to $\beta_{15} / \beta_{14}$, may be explained by a partial loss of selectivity from $V-V$ exchange and, probably, secondary chemical reactions. With irradiation at $938.7 \mathrm{~cm}^{-1}$, as one might expect from the spectral curves of excitation and dissociation (Figure $8.5 \mathrm{~b}$ and $8.5 \mathrm{c}$ ), preferential dissociation of $\mathrm{CH}_{3}{ }^{14} \mathrm{NO}_{2}$ was observed. The value of $K_{\mathrm{pr}}(14 / 15)$ was found to be $1.65 \pm 0.15$.

Similar results were obtained in experiments with $\mathrm{CF}_{2} \mathrm{Cl}_{2}{ }^{23}$ and $\mathrm{CF}_{3} \mathrm{Br}^{24}$ where isotopic selectivity of dissociation was also found from excitation of vibrations in the linear spectrum of which the isotope shift did not appear. In case of $\mathrm{CF}_{2} \mathrm{Cl}_{2}$ the separation of the chlorine isotopes ${ }^{35} \mathrm{Cl}$ and ${ }^{37} \mathrm{Cl}$ was achieved with the selectivity $\alpha=1.27\left(\Delta \nu_{\text {is }}\right.$ $\left.\left({ }^{35} \mathrm{Cl}-{ }^{37} \mathrm{Cl}\right)<0.2 \mathrm{~cm}^{-1}\right)$, and in the experiments with $\mathrm{CF}_{3} \mathrm{Br}$ it was possible to separate the bromine isotopes ${ }^{79} \mathrm{Br}$ and ${ }^{81} \mathrm{Br}$ with the selectivity $\alpha=1.04$. In analyzing these results one should take into account the fact that all vibrations including those in which an isotope shift manifests itself take part in the formation of a transition spectrum in the vibrational quasi-continuum. So the spectral characteristics of the quasi-continuum for molecules with different isotopes may somewhat differ. Furthermore, the density of states for the molecule with a heavier isotope must be also somewhat higher. All this can result in slightly different conditions for exciting molecules with different isotopes. It should be noted that at each of the successive transitions this difference may be very small. Nevertheless, it can bring about an appreciable difference in the value of the dissociation yield of two isotopic molecules. This will be illustrated in the following simple example.

Let us consider MP excitation of molecules assuming for simplicity that the excitation cross-section remains constant throughout the sequence of transitions, i.e., $\sigma_{j, j+1}=\sigma_{01}=$ const. In this case the probability of absorption of $n$ quanta by a molecule is given (see Section 3.2.2) by the Poisson distribution $w(n)=(\bar{n})^{n} \exp (-\bar{n}) / n|\cdot|$, where $\bar{n}$ is the average number of quanta absorbed by a molecular ensemble. In exciting two isotopes the difference in the average excitation level 
of these components will depend on the ratio of the transition crosssections

$$
\alpha_{\mathrm{ex}}=\frac{\overline{n_{i}}}{\overline{n_{k}}}=\frac{{ }^{i} \sigma_{01}}{{ }^{k} \sigma_{01}} \equiv \alpha_{01}
$$

When the difference in cross-sections is small, the excitation selectivity is small, too, i.e., $\left(\alpha_{01}-1\right) \ll 1$. But if the average molecular energy is much smaller than the dissociation energy, i.e., $\bar{n} \ll D / \hbar \Omega$, this small difference in excitation level will cause an appreciable difference in dissociation yield.

If a molecule must absorb $n$ quanta to dissociate, the probability ratio of this process for different isotopes, i.e., the selectivity of MP dissociation, will be $\alpha=\left(\alpha_{01}\right)^{n} \cdot \exp \left[\left(1-\alpha_{01}\right) \bar{n}\right]$. With $\left(\alpha_{01}-1\right) \bar{n} \ll 1$ the selectivity will be $\alpha \simeq \alpha_{01}^{n-\bar{n}}$. Thus, the effect can be increased considerably as compared to the simple ratio of absorption crosssections.

It must be stressed that such accumulation or multiplication of selectivity may take place only with $\beta \ll 1$ when a small fraction of molecules dissociates at the far tail of energy distribution $(n \gg \bar{n})$ which is very sensitive to the position of the distribution fuction maximum. We have already considered, in fact, this effect in Section 8.22 where the concept of excitation selectivity was introduced. Such multiplication can be alternatively explained by the accumulation of selectivity in a sequence of transitions if they are far from being saturated as actually takes place for the far distribution tail. The experiments with the molecule $\mathrm{UO}_{2}(\mathrm{hfacac})_{2} \cdot \mathrm{THF}^{4}$ are one of the striking demonstrations for such an effect of selectivity multiplication. The isotope shift in the linear spectrum of the $\nu_{3}$ vibration of these molecules for the isotopes ${ }^{235} \mathrm{U}$ and ${ }^{238} \mathrm{U}$ comes to $\Delta \nu_{\text {is }}=0.7 \mathrm{~cm}^{-1}$, the width of the spectrum being $8 \mathrm{~cm}^{-1}$. This molecule has a large number of vibrations and so, when it is under MP excitation in the $10.6 \mu$ region, all the transitions including the first one lie in the quasi-continuum, with the cross-section of successive transitions ${ }^{4}$ being almost constant. In this case the selectivity value for molecules having absorbed $n$ photons from the initial state with $\beta \ll 1$ actually comes to $\alpha \simeq\left(\alpha_{01}\right)^{n},{ }^{4}$ where $\alpha_{01}$ is the ratio of linear absorption cross-sections for the two isotopes. The comprehensive calculations carried out ${ }^{4}$ within the frameworks of such an approach agree well with the experimentally measured values of $\alpha$. 


\subsection{Ultimate spectral selectivity of MP dissociation. Gas temperature effect}

Spectral selectivity. The results given in the foregoing section confirm the conclusion that the isotopic selectivity of MP dissociation is related to the resonant properties of MP excitation. The study of the resonant characteristics of molecular excitation and dissociation enable us to conclude that even in the absence of channels of subsequent selectivity loss it must have a finite value. This is due to the fact that each of the isotopic modifications of a molecule has a finite width of the spectral dependence of dissociation yield. The degree of overlapping of these spectral dependences determines the ultimate spectral selectivity of MP dissociation. Such a conclusion is supported, for example, by experiments at low gas pressures when the possibility of selectivity loss resulting from molecular collisions is removed. In this case the experiments (see Section 8.5 ) show that the selectivity $\alpha$ within a low-pressure range tends to a finite value $\alpha_{0}$. It is quite natural ${ }^{25}$ to relate this value of $\alpha_{0}$ to the ultimate spectral value of selectivity that can be attained with purely radiative excitation.

The present understanding of the process of MP dissociation is that the resonant characteristics comes about mainly during excitation through low-lying vibrational states. Hence, the spectral selectivity of MP dissociation, too, is formed basically at the initial stage of molecular excitation by laser radiation. Very big molecules having a large number of vibrational modes and a low quasi-continuum limit are apparently an exception to this rule. This leads to certain specificity of excitation and formation of selectivity in these molecules (see below).

There is a close relation between the resonance characteristics of MP dissociation and the selectivity of this process, i.e., the action on the spectrum of MP excitation and dissociation of molecules. When the spectrum deformation comes about so that at a fixed frequency the dissociation yield for two isotopes changes in different ways, it evidently causes the selectivity to change. One such mechanism is through variations in the temperature of the gas under irradiation.

Temperature effect. The studies into the spectra of MP molecular dissociation (see Chapter 7) have shown that the form of these curves depends in many respects on the initial state of the molecules being excited. It has been shown ${ }^{26}$ for example, the large width of the spectral dependence of absorption in $\mathrm{SF}_{6}$ measured at room temperature 
$T=293 \mathrm{~K}$ is connected with the excitation of molecules from thermally populated low-lying vibrational states. In case of $\mathrm{SF}_{6}$ with $T=293 \mathrm{~K}$ only $\simeq 30 \%$ molecules are in the ground state, and so the contribution of "hot" bands to the formation of the excitation and dissociation spectra is considerable. To narrow the spectral contour of dissociation yield and to increase its selectivity it was proposed ${ }^{27}$ to use gas cooling, particularly when it flows through the nozzle. That was achieved in a number of subsequent experiments.

Let us consider the case of $\mathrm{SF}_{6}$. To enrich the dissociation products with the ${ }^{33} \mathrm{~S}$ isotope it is of course necessary to selective dissociate ${ }^{33} \mathrm{SF}_{6}$. It follows ${ }^{14}$ that because of the large width of the spectral contour of dissociation yield and a rather small isotope shift in the frequency of the $\nu_{3}$ vibration of ${ }^{32} \mathrm{SF}_{6},{ }^{33} \mathrm{SF}_{6}$ and ${ }^{34} \mathrm{SF}_{6}$ it is impossible to gain high dissociation selectivity of ${ }^{33} \mathrm{SF}_{6}$ at room temperature. The maximum simultaneously expected values of $\alpha(33 / 32)$ and $\alpha(33 / 34)$ (from the intersection of the curves in Figure 8.3) come to about 2. Therefore, to obtain high values of separation factor, cooling ${ }^{28}$ of $\mathrm{SF}_{6}$ was used. Figure 8.6. shows the spectral dependence of the dissociation yield of ${ }^{32} \mathrm{SF}_{6}$ with $T=173 \mathrm{~K}$. It might be seen that this curve differs significantly from the one measured at $T=293 \mathrm{~K}$ (see Figure 8.3). Specifically, the width of the contour and the amplitude of its longwave wing have decreased.

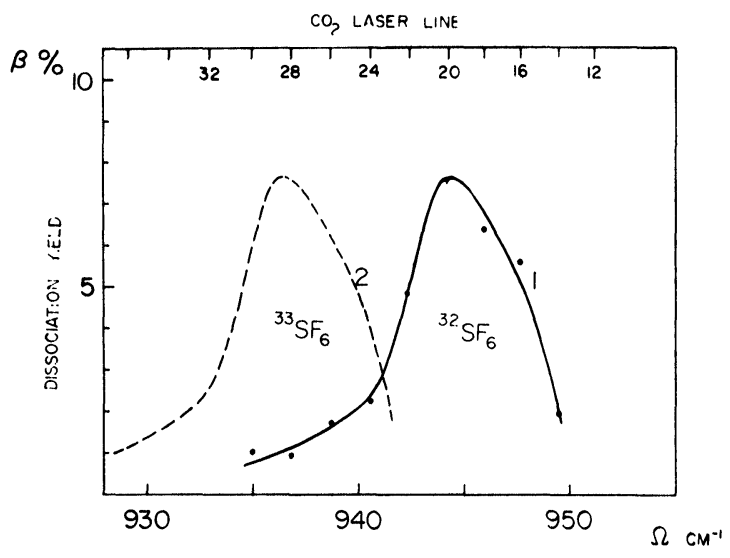

FIGURE 8.6 Spectral dependence of the dissociation yield of ${ }^{32} \mathrm{SF}_{6}$ (curve 1) at a gas temperature $T=173 \mathrm{~K} ; \Phi=5.4 \mathrm{~J} / \mathrm{cm}^{2}$. Dashed line (2) is the curve for ${ }^{33} \mathrm{SF}_{6}{ }^{28}$ 
The dashed line in Figure 8.6 also shows the dissociation contour for ${ }^{33} \mathrm{SF}_{6}$ produced by displacing the curve for ${ }^{32} \mathrm{SF}_{6}$ by $8 \mathrm{~cm}^{-1}$ which corresponds to the value of isotope shift. From the intersection of these curves the spectral dependence of $\alpha(33 / 32)$ was found (Figure 8.7). the experimental dependence of $K_{\mathrm{pr}}(33 / 32)$ is shown in the same figure. The maximum value of the enrichment factor in the products was $K_{\mathrm{pr}}(33 / 32)=5$. The value of selectivity found from relation (8.4) turned out to be equal to $\alpha_{\max }(33 / 32)=7$ which was close to its expected value.

Thus, the experiments with $\mathrm{SF}_{6}$ show that, as expected, gas cooling causes the spectral dependence of the dissociation yield to be narrowed which, in turn, increases the spectral selectivity of molecular dissociation.

In the above case of $\mathrm{SF}_{6}$ the dissociation contour width (at half height) at room temperature was larger than the isotope shift between the adjacent isotopes, almost twice as large in the experiment (Figure 8.3). Thus it was quite natural that the narrowing of the dissociation contour in gas cooling resulted in a considerable increase in selectivity. A decrease in temperature, however, involves an essential increase of

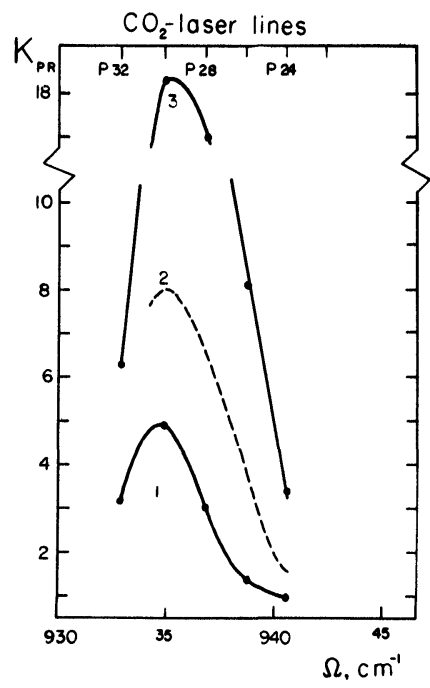

FIGURE 8.7 Enrichment factor $K_{\mathrm{pr}}$ in the dissociation products of $\mathrm{SF}_{6}$ as a function of laser excitation frequency with $T=173 \mathrm{~K}^{28}$ (1) $K_{\mathrm{pr}}(33 / 32)$; (2) calculated from the intersection of curves 1 and 2 in Figure 8.6; (3) $K_{\mathrm{pr}}(33 / 34)$. 
$\alpha$ even at large isotope shifts which exceed the dissociation contour width. $\mathrm{CF}_{3} \mathrm{I}$ is an example. In this case the isotope shift for ${ }^{12} \mathrm{CF}_{3} \mathrm{I}$ and ${ }^{13} \mathrm{CF}_{3} \mathrm{I}$ in the mode ${ }^{8}$ under excitation is $\Delta \nu_{\text {is }} \simeq 26 \mathrm{~cm}^{-1}$ which is larger than the dissociation contour width ${ }^{29} \Delta \nu \simeq 17 \mathrm{~cm}^{-1}$. In the first work on $\mathrm{CF}_{3} \mathrm{I}$ dissociation ${ }^{30}$ it was found that in the dissociation of ${ }^{12} \mathrm{CF}_{3} \mathrm{I}$ the value of $\alpha(12 / 13)$ increased with gas cooling. More detailed studies into the isotopic selectivity of $\mathrm{CF}_{3} \mathrm{I}$ dissociation ${ }^{8,25}$ show that the same effect can be also observed in the dissociation of ${ }^{13} \mathrm{CF}_{3} \mathrm{I}$. Figure 8.8 shows the dependence of $K_{\mathrm{pr}}(13 / 12)$ on gas temperature (the content of ${ }^{13} \mathrm{C} x_{0}=0.0108$ ). The measurements were taken with the $\mathrm{CF}_{3} \mathrm{I}$ pressure $p=1$ Torr when the value of $K_{\mathrm{pr}}$ was determined mainly by the spectral selectivity of excitation. It may be seen that the enrichment factor is highly dependent on temperature. As $T$ varies between $370 \mathrm{~K}$ and $230 \mathrm{~K}$, the value of $K_{\mathrm{pr}}(13 / 12)$ increases by 8 times.

The measurements of the spectral dependence of $\alpha(13 / 12)$ at two different gas temperatures $+22^{\circ} \mathrm{C}$ and $-50^{\circ} \mathrm{C}$ show ${ }^{31}$ that also in other molecules $\left(\mathrm{CF}_{3} \mathrm{Br}, \mathrm{CF}_{3} \mathrm{Cl}\right)$ the selectivity is higher with gas cooling.

The temperature dependence of selectivity may depend essentially on the conditions of excitation and on the laser radiation frequency in particular. In the above experiments with $\mathrm{CF}_{3} \mathrm{I}^{8,25}$ the ${ }^{13} \mathrm{CF}_{3} \mathrm{I}$ was excited near the center of the corresponding vibration $\nu_{1}$. The strong temperature dependence of $\alpha$ given in Figure 8.8 is caused only by

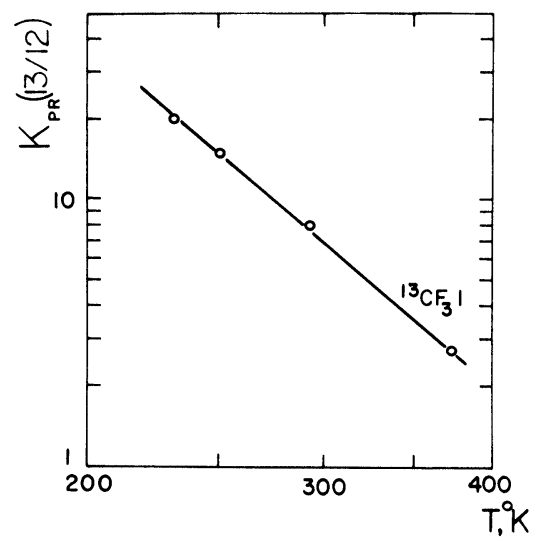

FIGURE 8.8 Enrichment factor in the dissociation products of ${ }^{13} \mathrm{CF}_{3} \mathrm{I} K_{\mathrm{pr}}(13 / 12)$ as a function of gas temperature. The pressure of $\mathrm{CF}_{3} \mathrm{I}$ is $1 \mathrm{Torr}$; the content of ${ }^{13} \mathrm{C}-x_{0}=$ $1.08 \%$. (On the basis of the data from Refs. 8 and 25 .) 
the change in the dissociation yield of nonresonant molecules ${ }^{12} \mathrm{CF}_{3} \mathrm{I}$ while the dissociation yield for ${ }^{13} \mathrm{CF}_{3} \mathrm{I}$ remains constant. At the same time, in the experiments with the hexafluoroacetone $\mathrm{CF}_{3}{ }^{12} \mathrm{COCF}_{3}$ and $\mathrm{CF}_{3}{ }^{13} \mathrm{COCF}_{3}{ }^{32}$ at decreasing temperature the dissociation yield for both isotopic species decreased. This decrease was slower for $\mathrm{CF}_{3}{ }^{13} \mathrm{COCF}_{3}$ in resonance with the field which resulted in an increase in selectivity. As the detuning from the absorption band of the both molecules was increased (to the red side), the dependences $\beta=\beta(T)$ became almost equal which caused $\alpha$ to be independent of $T$.

The effect of the conditions of excitation on the character of the dependence of $\alpha$ on $T$ is demonstrated more vividly by the experiments with $\mathrm{CF}_{2} \mathrm{Cl}_{2}{ }^{33}$ Figure 8.9 shows the dependence of the $\alpha(12 / 13)$ selectivity on temperature with excitation at the lines $P(24)$ (the edge of the $R$ branch of the $\nu_{8}$ mode $\left(923 \mathrm{~cm}^{-1}\right)$ of $\left.{ }^{12} \mathrm{CF}_{2} \mathrm{Cl}_{2}\right)$ and $P(38)$ (around the center of the $R$ branch). It may be seen that at a strong blue shift from the both isotopes $(P(24)$ line) there is even an increase in $\alpha$ as the temperature rises. At the $P(38)$ line $\alpha$ varies slightly with $T$.

The dependences of MP dissociation selctivity on temperature can be caused by different factors. As noted above, the formation of the

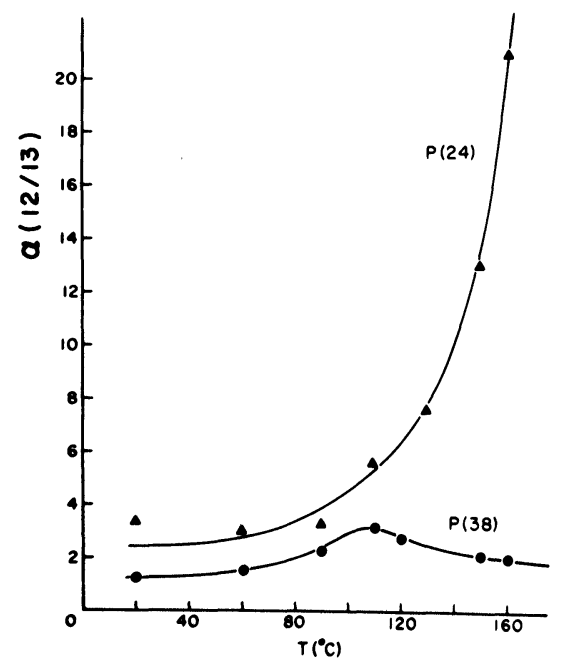

FIGURE 8.9 Dependence of the dissociation selectivity of $\mathrm{CF}_{2} \mathrm{Cl}_{2} \alpha(12 / 13)$ on gas temperature at the $P(38)$ and $P(24)$ lines of the $10.6 \mu$ band of the $\mathrm{CO}_{2}$ laser. 
MP excitation spectrum at lower transitions, and particularly during excitation of molecules with a large shift from the center of the band, is still not clearly understood. We can just present here some qualitative considerations.

Variations in temperature bring about the change in population of both rotational and low-lying vibrational levels. In the first case the variation of the rotational distribution can change the fraction of molecules interacting with the radiation and, as a result, affect the value of dissociation yield and hence selectivity. In the second case, as the vibrational distribution varies, the initial vibrational molecular energy changes. As a consequence, it is easier to excite the molecules to the quasi-continuum since the distance to its "limit" is reduced. It is obvious that this process can also affect selectivity. It is difficult to say which of these factors has a dominant role in the above experiments. It is not improbable that there are some other factors causing $\alpha$ to be dependent on temperature.

The picture is more clear in the excitation of big molecules. In this case the temperature dependence of selectivity is caused mainly by the temperature dependence of the energy gap between the molecular energy content and the dissociation energy limit. The large number of vibrational degrees of freedom in these molecules enables them even at room temperature to store vibrational energy that accounts for a considerable portion, about one half of the dissociation energy. This takes place, for example, in case of $\mathrm{UO}_{2}(\mathrm{hfacac})_{2} \cdot \mathrm{THF}$. Thus even a slight variation in temperature considerably changes the initial molecular energy which is equivalent to the change in position of the dissociation limit. For molecules of such a type $\alpha \simeq\left(\alpha_{01}\right)^{n}$ (see Section 8.2.3), where $n$ is the number of quanta which must be absorbed for dissociation. It is evident that a decrease in temperature involves an increase in $n$ and hence an increase in selectivity. A more detailed calculation within such a model ${ }^{4}$ with allowance made for the initial vibrational distribution of molecules and the final lifetime relative to dissociation agrees well with experiment.

Thus, the results presented show that gas temperature can essentially affect the selectivity of molecular dissociation. Under certain conditions variation in temperature may serve as an effective means to increase selectivity which is of great importance for a number of applications. In conclusion it should be noted that variation in temperature leads not only to a change of the ultimate spectral selectivity 
of MP dissociation but it can also affect the character of the dependence of $\alpha$ on gas pressure. This point will be considered in more detail in Section 8.5.

\subsection{Role of laser pulse intensity. Two-frequency MP dissociation}

Intensity effects. Studies of MP dissociation show that the resonant characteristics of this process, particularly the form of the spectral dissociation contour, its width and position, depend on laser radiation intensity. This, of course, must cause the selectivity to depend on exciting radiation intensity since its spectral value is governed by the resonant properties of dissociation.

Moreover, several studies on the spectra of multiphoton excitation of molecules by intense IR radiation show that with increasing intensity they are broadened and the structure, if exists, diffuses. Therefore, irrespective of what factors are responsible for these changes-new multiphoton resonances, weak transitions, power broadening, etc.one should expect that an increase in radiation intensity will bring about a decrease in selectivity.

There is a number of experiments in which the dependence of selectivity on radiation intensity has been studied. The first experiments $^{34}$ were the dissociation of $\mathrm{SF}_{6}$ by focusing a laser pulse $1 \mathrm{~ns}$ long, with a peak radiation intensity of $10^{13} \mathrm{~W} / \mathrm{cm}^{2}$. There was no selectivity found. This would be expected since under the conditions of the experiment the power broadening $\gamma_{01}$ was much larger than the value of isotope shift $\Delta \nu_{\text {is }}(32 / 34)=17 \mathrm{~cm}^{-1}$.

Of great interest are experiments under much "softer" excitation, at least when $\gamma_{01}<\Delta \nu_{\text {is }}$. Here attention must be drawn to the experiments with a different laser pulse shape. ${ }^{35}$ In this work $\mathrm{SF}_{6}$ was irradiated in two ways. In the first case the $\mathrm{CO}_{2}$ laser pulse was smooth in shape and in the second case almost $100 \%$ modulation of radiation intensity was observed as a result of self mode locking. The energy in the both cases was constant and so the peak radiation intensity in the second case was higher. The $\mathrm{CO}_{2}$ laser radiation frequency was tuned to the absorption band of ${ }^{32} \mathrm{SF}_{6}$. The dissociation selectivity of ${ }^{32} \mathrm{SF}_{6}$ with relation to ${ }^{33} \mathrm{SF}_{6}$ was found to be higher by $18 \%$ in case of irradiating with a smooth pulse. The isotope shift in this case was $\Delta \nu_{\text {is }}(32 / 33) \simeq 8 \mathrm{~cm}^{-1}$. The difference in the enrichment factor relative to ${ }^{34} \mathrm{SF}_{6}$, with $\Delta \nu_{\text {is }}$ twice as high, could not be observed. 
So this experiment shows that an increase in intensity causes the dissociation selectivity to decrease, this effect increasing as the isotope shift is reduced. It is difficult, however, to carry out quantitative interpretation because the measurements were taken at high pressures of $\mathrm{SF}_{6}$ when an important contribution to $\alpha$ was made by the interisotopic $V-V$ exchange and so purely field effects were considerably reduced.

Quantitative information on the character of the dependence of selectivity on radiation intensity were obtained ${ }^{36}$ in experiments where a mixture of $\mathrm{CF}_{3} \mathrm{CHCl}_{2}$ and $\mathrm{CF}_{3} \mathrm{CDCl}_{2}$ was irradiated at a total pressure of 0.5 Torr with a $\mathrm{CO}_{2}$ laser tuned to the absorption band of $\mathrm{CF}_{3} \mathrm{CDCl}_{2}$ whose concentration was $0.5 \%$. Figure 8.10 shows the dependence of the enrichment factor in the products $K_{\mathrm{pr}}(D / H)$ on energy fluence $\Phi$ (the pulse duration was $100 \mathrm{~ns}$ ). It may be seen that the dependence is rather sharp. As $\Phi$ was changed by an order of magnitude from $10 \mathrm{~J} / \mathrm{cm}^{2}$ to $100 \mathrm{~J} / \mathrm{cm}^{2}$, the value of $K_{\mathrm{pr}}(D / H)$ decreased from 1400 to 27 . In the same experiment the values of $\beta_{H}$ and $\beta_{D}$ were measured independently as pure $\mathrm{CH}_{3} \mathrm{CHCl}_{2}$ or $\mathrm{CF}_{3} \mathrm{CDCl}_{2}$ were irradiated. Their ratio $\beta_{D} / \beta_{H}$ agrees well with the

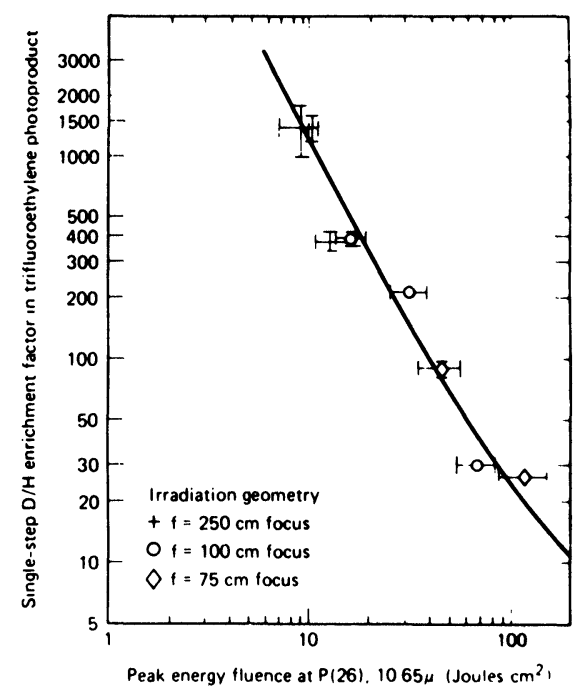

FIGURE 8.10 Enrichment factor in the products $K_{\mathrm{pr}}(D / H)$ as a function of pulse energy fluence in the dissociation of $\mathrm{CF}_{3} \mathrm{CDCl}_{2}{ }^{36}{ }^{36}$ The pressure of $\mathrm{CF}_{3} \mathrm{CHCl}_{2}$ is $0.5 \mathrm{Torr}$, the content of $\mathrm{CF}_{3} \mathrm{CDCl}_{2}$ is $0.5 \%$. 
measured value of $K_{\mathrm{pr}}(D / H)$. This supports the conclusion that the role of interisotopic $V-V$ exchange is small and the curve in Figure 8.10 denotes the dependence of $K_{\mathrm{pr}}$ on intensity in "pure" form, i.e. it reflects the character of the dependence of the spectral MP dissociation selectivity of $\mathrm{CF}_{3} \mathrm{CDCl}_{2}$ on radiation intensity. A decrease in dissociation selectivity has been also observed in experiments with the $\left(\mathrm{CH}_{3}\right)_{2} \mathrm{O}^{37}$

The results of the studies carried out so far into the process of MP molecular dissociation enable us to conclude that the finite value of dissociation selectivity of molecules with a chosen isotope is related to the laser excitation of molecules of a different isotopic composition. An increase in radiation intensity in general causes the excitation of the nondesired isotope to be increased stronger as compared with the desired one and hence the dissociation selectivity is decreased. One of the factors responsible for this may be the power broadening of the transition spectrum. This is not the only cause, however, since in the experiments with $\mathrm{CF}_{3} \mathrm{I}^{8,30}$ appreciable excitation of molecules with a different isotope can be also observed with $\Delta \nu_{\text {is }} \gg \gamma_{01}$.

At present there is no reliable theoretical description of MP excitation and dissociation spectra for specific molecules, and so it is difficult to carry out quantitative interpretation of the observed dependences of dissociation selectivity on exciting radiation intensity. At the same time the results obtained point to one more way of increasing spectral selectivity, that is to decrease the laser radiation intensity. Naturally it is desirable not to lose dissociation yield. These two requirements can be met in two-frequency molecular dissociation.

Molecular dissociation in a two-frequency field. In such a method of molecular dissociation the radiation at one frequency performs preliminary isotopically selective excitation of the molecules. It is mainly here that there is spectral selectivity of the MP dissociation. Subsequent excitation and dissociation of the molecules come about as a result of energy absorption from the field at another, usually displaced, frequency. The total radiation energy being the same, the division of the radiation into two pulses with different frequencies can considerably increase the dissociation yield as compared to the case of onefrequency irradiation. At the same time, as the molecules are excited at the first stage, a decrease in radiation intensity causes the dissociation contour to get narrow. Furthermore, it may cause, as in the case of $\mathrm{OsO}_{4}$, quite a distinct structure. Such behavior of the excitation 
and dissociation spectrum, the formation of a structure in it in particular, enables us to hope for a substantial increase in spectral dissociation selectivity, as compared to the one-frequency method, especially in separating isotopes of heavy elements when the isotope shift in the IR absorption spectrum is small.

This conclusion is confirmed by the experiments with $\mathrm{OsO}_{4}{ }^{38,39}$ performed with the natural abundance of osmium isotopes. The dissociation selectivity of $\mathrm{OsO}_{4}$ in a two-frequency field was studied with the same radiation intensity, $10^{7} \mathrm{~W} / \mathrm{cm}^{2}$, at both frequencies $\Omega_{1}$ and $\Omega_{2}$. The delay between pulses was $\tau \leqslant 10^{-6} \mathrm{~s}$. The dissociation of $\mathrm{OsO}_{4}$ was performed at different frequencies of exciting radiation $\Omega_{1}$. The frequency of dissociating radiation $\Omega_{2}$ was constant and corresponded to the $P(20)$ line of the $10.6 \mu$ band of $\mathrm{CO}_{2}$ laser.

Table 8.2 contains the results of mass-spectroscopic analysis of the residual gas $\mathrm{OsO}_{4}$ during its dissociation in a two-frequency field. The mixture under irradiation comprised $0.2 \mathrm{Torr}^{\mathrm{OsO}}{ }_{4}+2$ Torr OCS. The total dissociation of $\mathrm{OsO}_{4}$ throughout the irradiation time was $\simeq 90 \%$. It may be seen that maximum enrichment occurs when the $\Omega_{1}$ frequency is tuned to the $P(6)$ line. When $\Omega_{1}$ is tuned to the $P(20)$ line, preferential dissociation of ${ }^{188} \mathrm{OsO}_{4}$ was observed.

Table 8.2 also gives the results of isotope-selective dissociation of $\mathrm{OsO}_{4}$ in a one-frequency field. ${ }^{40}$ The total dissociation of $\mathrm{OsO}_{4}$ in this case was almost the same, that is, $90 \%$. The comparison of the results of irradiation in one- and two-frequency fields shows that in the second case the selectivity is higher. It should be noted that under twofrequency irradiation the $\mathrm{OsO}_{4}$ molecules had the natural abundance of Os isotopes. Specifically, the concentration ratio of ${ }^{192} \mathrm{Os}$ and ${ }^{187} \mathrm{Os}$ was $41: 1.64$. Under one-frequency irradiation these isotopes had the same abundance, and the maximum enrichment came to $K_{\text {res }}(192 / 187)=1.15$. The abundance of the isotopes in the mixture was natural and no enrichment was observed in the residual gas.

As in the ordinary method of excitation, in the case of two-frequency excitation the MP dissociation selectivity depends on radiation intensity and additionally on the intensity ratio between the first and second fields. This is confirmed by experiments with $\mathrm{SF}_{6} \cdot{ }^{41}$ The $\mathrm{SF}_{6}$ was excited by the first field at $\Omega_{1}=947.7 \mathrm{~cm}^{-1}$ (the $P(16)$ line of $\mathrm{CO}_{2}$ laser). The frequency of the second field was $\Omega_{2}=938.8 \mathrm{~cm}^{-1}$ (the $P(28)$ line). The dependences of the enrichment factor in the products $K_{\mathrm{pr}}(32 / 34)$ on the energy fluence $\Phi_{1}$ of the first field with $\Phi_{2}=$ const 


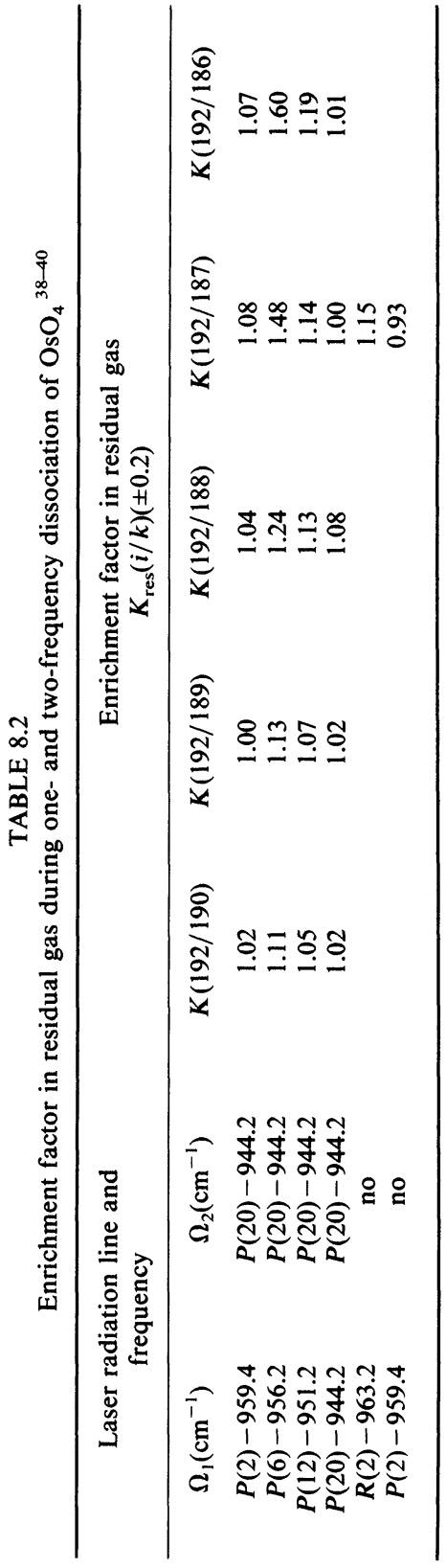


as well as on $\Phi_{2}$ with $\Phi_{1}=$ const are given respectively in Figure 8.11a and $8.11 \mathrm{~b}$. The initial decrease of $K_{\mathrm{pr}}$ (Figure $8.11 \mathrm{a}$ ), as the first field was engaged, was related to interisotopic $V-V$ exchange since the experiment was performed with $p=0.2$ Torr and the delay between pulses $\varepsilon=200 \mathrm{~ns}$ when the contribution of this process was already appreciable. A subsequent increase in $\Phi_{1}$ does not lead to any appreciable changes in selectivity.

The dependence $K_{\mathrm{pr}}=\bar{K}_{\mathrm{pr}}\left(\Phi_{2}\right)$ is quite different at fixed $\Phi_{1}$ (Figure $8.11 \mathrm{~b})$. The dissociation selectivity here rises monotonicly as the energy fluence of the second field increases. By the time the $\Omega_{2}$ pulse arrives there are two groups of excited molecules: the more excited ${ }^{32} \mathrm{SF}_{6}$ molecules and the less excited ${ }^{34} \mathrm{SF}_{6}$ molecules. The results obtained show that with increasing $\Phi_{2}$ the molecules of the first group absorb energy faster than those of the second group.

The application of the two-frequency dissociation technique essentially widens the scope of MP molecular dissociation. Two-frequency irradiation, for example, allows using rather low-power tunable lasers at the first step which will provide the wanted spectral selectivity of excitation. At the second step it is possible to use more powerful lasers which need not be tuned to the vibrational transitions of the molecule being excited.

This can be illustrated by the experiments on isotope separation of carbon and selenium in two-frequency dissociation of $\mathrm{CCl}_{4}{ }^{42}$ and $\mathrm{SeF}_{6}{ }^{43}$ respectively. In these experiments selective excitation of molecules with the desired isotope was carried out with a pulsed $\mathrm{NH}_{3}$ laser tuned to the frequency of one of the fundamental vibrations of $\mathrm{CCl}_{4}$ or $\mathrm{SeF}_{6}$. At the second step a pulsed $\mathrm{CO}_{2}$ laser was used. Its

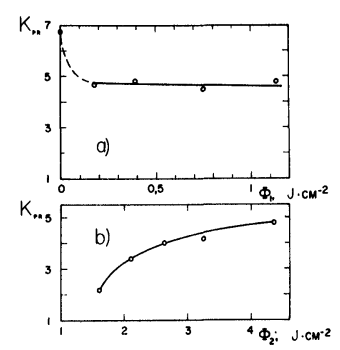

FIGURE 8.11 Enrichment factor $K_{\mathrm{pr}}(32 / 34)$ of $\mathrm{SF}_{6}$ as a function of laser energy fluence of the first pulse $\Phi_{1}$ with $\Phi_{2}=4.4 \mathrm{~J} / \mathrm{cm}^{2}$ (a) and the second pulse $\Phi_{2}$ with $\Phi_{1}=1 \mathrm{~J} / \mathrm{cm}^{2}(\mathrm{~b})$. 
radiation was out of resonance with the fundamental frequencies of vibrational transitions of these molecules.

As the experiments with $\mathrm{SeF}_{6}$ show, the addition of $\mathrm{CO}_{2}$ laser radiation provides a considerable increase in dissociation yield, by 2.5 times without a reduction in selectivity as compared to irradiation by only a $\mathrm{NH}_{3}$ laser. This result complies with the concept of the separate functions of the two fields according to which it is the first resonant field that is responsible for the spectral selectivity of excitation.

When using the two-frequency method of dissociation one should remember an important factor. As it has been noted and the direct experiments on two-frequency dissociation of $\mathrm{SF}_{6}$ show, ${ }^{44}$ the dissociation selectivity materially depends on the delay time between pulses $\tau$. With increasing $\tau$ the value of $\alpha(32 / 34)$ in experiments with $\mathrm{SF}_{6}$ decreased fast which the authors ${ }^{44}$ attributed to the excitation of ${ }^{34} \mathrm{SF}_{6}$ between pulses as a result of the process of $V-V$ exchange. Therefore, it is necesary to choose properly the delay time between the two fields in order to eliminate a considerable decrease of the selectivity attained at the first step of radiative excitation.

In conclusion we will mention another version of the two-frequency method of molecular dissociation. It includes the use of UV lasers at the second step as a source of dissociating radiation, with the preliminary multiple photon excitation at the first step by IR radiation. Experiments of this kind were performed with $\mathrm{CF}_{3} \mathrm{I}^{45} \dagger$ The isotopeselective excitation at the first step was performed with $\mathrm{CO}_{2}$ laser radiation. The excited molecules were dissociated with $\mathrm{XeF}$ laser radiation at the $350 \mathrm{~nm}$ wavelength. The value of selectivity obtained in these experiments $\alpha(12 / 13)=48$ exceeds the values resulting from one-frequency MP dissociation of $\mathrm{CF}_{3} \mathrm{I}$ by IR radiation at the same temperature and gas pressure. This points to great potential for the method of double IR-UV molecular photodissociation.

\subsection{Effects of collisions on the selectivity of MP dissociation}

In discussing the effect of isotopic selectivity of MP dissociation we have so far neglected the effect of collisions. In most cases this is

† These experiments are further development of the method of double IR-UV dissociation discussed in Refs. 46 and 47. 
possible in experiments at sufficiently low gas pressures. But very often, particularly in different applications it becomes necessary to excite molecules at gas pressures as high as possible. The study of collisional processes for highly excited molecules is also of independent interest. A large number of research thus deal with the effect of collisions on isotopic selectivity.

An increase in gas pressure leads to a rather great number of collisions taking place during MP dissociation, i.e., in the time in which the basic fraction of dissociating molecules decays. $\dagger$ As a result of these collisions, the vibrational and rotational states of colliding partners change. In the previous chapters it has been shown that the processes of $V-V, V-T$ and rotational relaxation may have a pronounced and, under certain conditions, a very significant effect on the process of MP dissociation and the observed dissociation yield. In the case of an excitation of a two-component medium the effect of collisions on selectivity is reduced to the different action of multicomponent collisional processes on MP dissociation. There are very many possibilities of these effects, however.

The existence of several types of relaxation processes, the difference in rates of these processes (see Section 2.5) cause the effect of collisions on selectivity to be highly dependent not only of the conditions of excitation but also of the properties of the molecule being excited and the type of collision partner. All this makes the observed effects varied.

This section presents the essential results obtained in studying the influence of collisions on MP dissociation selectivity. While describing these results we tried to systematize them from the standpoint of the contribution of different relaxation processes so far as the available data allowed. It should be noted, however, that the role of each has not been studied nearly in equal measure. If the action of interisotopic $V-V$ exchange, at least during a radiation pulse, is understood quite sufficiently, this cannot be said about $V-T$ and rotational relaxations.

We are going to begin by describing the results of studies into the effect of the parent gas pressure on selectivity.

\subsubsection{Dependence of selectivity on parent gas pressure and relative isotope content}

As can be seen from the discussion below, the effect of the pressure of the gas under irradiation on MP dissociation selectivity may

$\dagger$ This time may apparently exceed the exciting pulse duration. 
materially depend on the relative concentration $x_{0}$ of the isotopic component being excited. Therefore, we shall consider separately the cases of exciting components with high $\left(\left(1-x_{0}\right) \ll 1\right)$ and low $\left(x_{0} \ll 1\right)$ contents.

Excitation of a "rich" component $\left(\left(1-x_{0}\right) \ll 1\right)$

The dependence of the MP dissociation selectivity on parent gas pressure in this case has been studied in many experiments for different molecules and isotopes. The characteristic feature of the dependences produced is decreasing selectivity as the gas pressure increases starting, at least, with a certain characteristic pressure. A monotonic decrease in dissociation selectivity with an increase in parent gas pressure has been observed in experiments with $\mathrm{SF}_{6},{ }^{14,17} \mathrm{BCl}_{3},{ }^{9,34} \mathrm{CF}_{2} \mathrm{Cl}_{2}{ }^{34}$ $\mathrm{HCOOH},{ }^{48}$ and $\mathrm{CF}_{3} \mathrm{I}^{8,30}$

Figure 8.12 shows the dependence of $K_{\mathrm{pr}}(32 / 34)$ attained in the dissociation of ${ }^{32} \mathrm{SF}_{6} .{ }^{14}$ As the pressure of $\mathrm{SF}_{6}$ increases, $K_{\mathrm{pr}}$ drops exponentially. The characteristic pressure $\rho_{0}$ at which $K_{\mathrm{pr}}$ is reduced by $e$ times comes to 0.55 Torr.

In most cases such a decrease in selectivity with increasing gas pressure is attributed to the process of $V-V$ exchange, as a result of which transfer of vibrational energy to unexcited nonresonant isotopic molecules takes place during collisions. This causes the dissociation of these molecules to increase due to two factors. First, the molecules with the undesired isotope excited in $V-V$ exchange are able to dissociate during a laser pulse. In more detail this case is considered in Section 8.5.2 where the quantitative relations connecting the $\alpha$ value with the $V-V$ exchange rate constant, gas pressure and laser pulse duration are also presented.

Secondly, the excitation of these molecules by $V-V$ exchange after a laser pulse can make a considerable contribution to increasing the

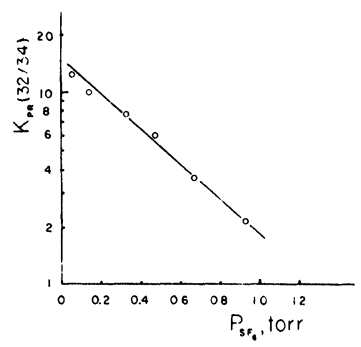

FIGURE 8.12 Enrichment factor $K_{\mathrm{pr}}(32 / 34)$ as a function of the pressure of $\mathrm{SF}_{6} \cdot{ }^{14}$ 
undesired isotope dissociation yield. In both cases $V-V$ exchange causes the dissociation selectivity to decrease with increasing pressure.

There is one more factor, in principle, that makes $\alpha$ depend on parent gas pressure. It is the decrease in dissociation yield $\beta$ of the desired isotope as the pressure rises and, for example, may take place when a considerable contribution to $\beta$ is made by the dissociation of molecules in long-lived states above the dissociation limit. The energy exchange between the unexcited molecules and the ensemble of highly excited molecules causes the latter to de-excite and both $\beta$ and $\alpha$ to decrease.

The decrease of $\beta$ for the desired isotope was pointed to as one of the factors responsible for the decrease of selectivity with increasing pressure. ${ }^{14,35}$ But it should be noted that the $V-V$ exchange with the molecules of the "cold" ensemble brings about the de-excitation and decrease of the dissociation yield for the molecules with the undesired isotope, too. So the sign of selectivity variation depends on the ratio of the contributions of this process to the dissociation yield for two isotopes. Probably, situations are not impossible when such a process may result in an increase in selectivity.

The value of enrichment factor in residual gas, as shown in many experiments, ${ }^{9,27,34,49}$ is also reduced as the gas pressure increases. Such behaviour of $K_{\text {res }}$ results from the dependence of this value both on the selectivity $\alpha$ and the dissociation yield $\beta$ of the molecules with the isotope under excitation (see (8.4)). An increase in pressure involves a decrease in $\alpha$ and, besides, can cause $\beta$ to decrease, as it has been just noted. It is these two factors that cause the enrichment factor $K_{\text {res }}$ to decrease as the gas pressure increases.

Excitation of a "poor" component $\left(x_{0} \ll 1\right)$ In this case the excited molecules with the poor isotope are surrounded by molecules with the rich isotope and actually only collide with them. This may bring about quite definite differences in the dissociation conditions of both components, as compared to the case of the excitation of the rich isotope and, as a result, there is a difference in the behavior of the dependence of $\alpha$ on $\rho$. The experiments confirm this conclusion.

Figure 8.13a shows the dependence of the enrichment factor $K_{\mathrm{pr}}$ in the products on the total gas pressure $\rho$ of $\mathrm{CF}_{3} \mathrm{I}$ when ${ }^{13} \mathrm{CF}_{3} \mathrm{I}$ with the natural abundance of the ${ }^{13} \mathrm{C}$ isotope $x_{0}=0.0108$ is excited at two temperatures. For comparison, Figure $8.13 \mathrm{~b}$ presents analogous depen- 


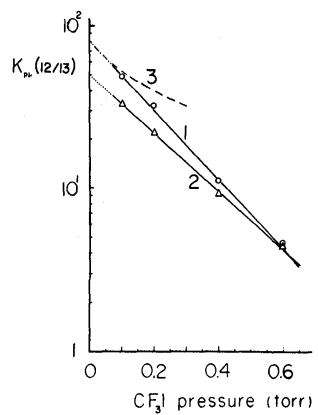

FIGURE 8.13a Enrichment factor in the dissociation products of ${ }^{12} \mathrm{CF}_{3} I K_{\mathrm{pr}}(12 / 13)$ as a function of the total pressure of $\mathrm{CF}_{3} \mathrm{I}^{25}$ Curves: (1) $T=-45^{\circ} \mathrm{C}$; (2) $T=+20^{\circ} \mathrm{C}$; (3) calculations (see Section 8.5.2).

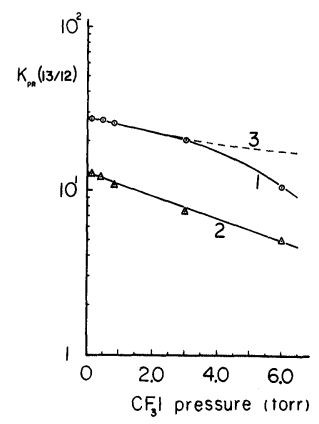

FIGURE 8.13b Enrichment factor in the dissociation products of ${ }^{13} \mathrm{CF}_{3} \mathrm{I} K_{\mathrm{pr}}(13 / 12)$ as a function of the total pressure of $\mathrm{CF}_{3} \mathrm{I}^{25}$ Curves: (1) $T=-45^{\circ} \mathrm{C}$; (2) $T=+20^{\circ} \mathrm{C}$; (3) calculations.

dences for the case of excitation of ${ }^{12} \mathrm{CF}_{3} \mathrm{I}$ under the same experimental conditions. In both cases the value of $K_{\mathrm{pr}}$ is decreased as the pressure rises. This decrease can be described, as in the case of $\mathrm{SF}_{6}$ (see Figure $8.12)$, by an expression like $K_{\mathrm{pr}}=K_{\mathrm{pr}}^{0} \exp \left(-\rho / \rho_{0}\right)$. The values of $K_{\mathrm{pr}}^{0}$ and $\rho_{0}$ are given in Table 8.3. One can clearly see that the dependence of $K_{\mathrm{pr}}$ on $\rho$ in the case of ${ }^{13} \mathrm{CF}_{3} \mathrm{I}$ is much slower. In this case $\rho_{0}$ is 53 times higher (the gas temperature $T=-45^{\circ} \mathrm{C}$ ) than that of ${ }^{12} \mathrm{CF}_{3} \mathrm{I}$, and so the value of $K_{\mathrm{pr}}$ remains high even at comparatively high gas pressures. ( $K_{\mathrm{pr}}=20$ with $p=3$ Torr).

A slower dependence of selectivity on gas pressure in exciting molecules with the poor isotope was observed also in the experiments 
with the natural mixture of ${ }^{13} \mathrm{CF}_{2} \mathrm{Cl}_{2}$ and ${ }^{12} \mathrm{CF}_{2} \mathrm{CL}_{2} \cdot{ }^{50}$ High selectivity at high gas pressures was also obtained in the experiments with $\mathrm{CF}_{3} \mathrm{CHCl}_{2} \cdot{ }^{36}$ As the deuterated molecule $\mathrm{CF}_{3} \mathrm{CDCl}_{2}\left(x_{0}=0.005\right)$ was excited by $\mathrm{CO}_{2}$ laser pulses $100 \mathrm{~ns}$ long, the enrichment factor in the products was $K_{\mathrm{pr}}=4$ with $p=25$ Torr.

Such behavior of the dependence of selectivity on pressure at different concentrations of the isotope being excited that seems to be unexpected at first sight has rather a simple physical explanation. As mentioned above, a decrease in selectivity with increasing pressure comes about due to increasing rate of $V-V$ exchange. The probability of the molecule with the undesired isotope acquiring necessary excitation in such a process and dissociating is proportional to the number of collisions with the excited molecules containing the desired isotope and hence to their partial pressures. Thus, dissociation selectivity must be determined by the partial pressure of the molecules with the isotope under excitation. ${ }^{8}$ As a result, when the concentration of resonart isotope varies, it leads to changes in the rate of pressure dependence of selectivity. The conditions under which this conclusion is valid (in the case $x_{0} \ll 1$ ) will be considered in Section 8.5.2.

In the case considered above the decrease in selectivity with an increase in the total gas pressure was slow but quite definite. However, there may be an increase in selectivity as the pressure increases. Such an increase in $\alpha$ was observed in the experiments on hexafluoroacetone. ${ }^{51}$ Figure 8.14 shows the dependence of dissociation selectivity in exciting $\mathrm{CF}_{3}{ }^{13} \mathrm{COCF}_{3}$ with the natural abundance of ${ }^{13} \mathrm{C}$. As the total gas pressure increases up to 10 Torr, the selectivity is increased by about 5 times. An increase in selectivity with increasing gas pressure was also observed in experiments with $\mathrm{CF}_{3} \mathrm{Br}^{52,53}$ In this case, too, $\alpha$ increased as the component with a low content of ${ }^{13} \mathrm{C}$, ${ }^{13} \mathrm{CF}_{3} \mathrm{Br}$, was excited.

An important specific feature that unites these experiments and differentiates them from those considered where $\alpha$ decreases with increasing $\rho$ is a large detuning from the center of the MP excitation band of the desired isotope. The dependence of $\alpha$ on $p$ shown in Figure 8.14, for example, is obtained with the detuning (to the red side) from the center of the band of $\mathrm{CF}_{3}{ }^{13} \mathrm{COCF}_{3}$ being $31 \mathrm{~cm}^{-1}$, its total width $\leqslant 7 \mathrm{~cm}^{-1}$. ${ }^{51}$ The detuning from the band of $\mathrm{CF}_{3}{ }^{12} \mathrm{COCF}_{3}$ in this case comes to $47 \mathrm{~cm}^{-1}$. The excitation at the far end of the MP absorption band is apparently one of the critical conditions for 


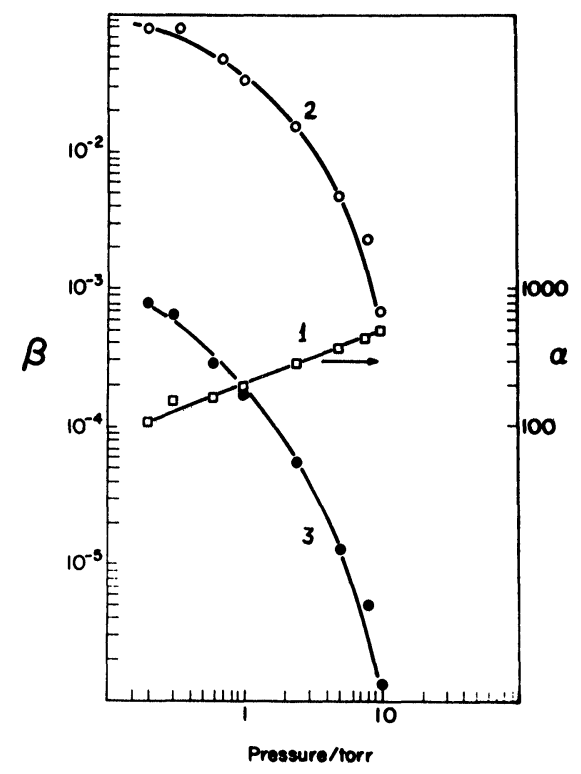

FIGURE 8.14 Isotopically selective MP dissociation of hexafluorineacetone. ${ }^{51}$ Excitation of $\mathrm{CF}_{3}{ }^{13} \mathrm{COCF}_{3}$. Curve 1 is the selectivity of $\mathrm{MP}$ dissociation. Curves 2 and 3 denote the dissociation yields of $\mathrm{CF}_{3}{ }^{13} \mathrm{COCF}_{3}$ and $\mathrm{CF}_{3}{ }^{12} \mathrm{COCF}_{3}$ respectively.

observing an increase in $\alpha$. This is confirmed by experiments with $\mathrm{CF}_{3} \mathrm{Br}^{53}$ (see also Section 8.5.3, Figure 8.16), where is illustrated that the dependence of $\alpha$ on $\rho$ varies with the laser radiation frequency. With excitation at the center of the band the selectivity decreases with increasing gas pressure.

The results will be discussed in more detail below, at the end of Sections 8.5.2 and 8.5.3.

\subsubsection{Role of $V-V$ exchange}

The results of experiments at different pressures of the gas being irradiated show that the $V-V$ exchange bringing about vibrational excitation and dissociation of molecules with the undesired isotope may have a pronounced effect on the selectivity of MP dissociation. As a result, the measured values of selectivity become, as a rule, smaller than their spectral value. 
The process of $V-V$ exchange during MP molecular dissociation may be divided into two stages: exchange during an exciting pulse and after the pulse is over. This division is natural and clearly during the laser pulse there is a source of molecular excitation (the laser) which can compensate for the energy flow from the resonant component caused by a $V-V$ process. This is especially essential in exciting the lesser component. For lack of sufficient data the criterion-at which of the stages the $V-V$ exchange gives the greatest contribution to the decrease of selectivity-has not been formulated exactly yet. Therefore we will restrict ourselves to a general note. It is quite evident that, when a great part of dissociating molecules decays during an exciting pulse, the first stage of $V-V$ exchange is the main cause of any selectivity variation.

The results are given below of studies into the effect of $V-V$ exchange on the isotopic selectivity of MP dissociation. First we shall consider comprehensively the first stage, that is, the $V-V$ exchange during a laser pulse, and then touch upon its contribution after the laser pulse is over.

\section{Model of $V-V$ exchange during a laser pulse}

First we are going to consider the model of $V-V$ exchange developed in Ref. 25 to explain experiments on isotopically selective dissociation of $\mathrm{CF}_{3} \mathrm{I}$ molecules. The results obtained, however, are general in character and allow quantitative analysis of the role of $V-V$ exchange during excitation and dissociation of molecules in a strong IR field.

In this model it is assumed that a decrease in spectral selectivity results from the vibrational excitation exchange in collisions of molecules with different isotopes during a laser pulse. As a result of this process, the molecules with the undesired isotope whose vibrational transition frequency is out of resonance with the radiation can acquire sufficient energy for their subsequent excitation and dissociation by laser radiation.

In a real situation the colliding molecules may be in different energy states, and the exchange rate may depend on the excitation level of these molecules. In the model in use the processes of exchange and laser excitation are considered in a simplified manner. The molecules excited to the vibrational quasi-continuum are regarded as one ensemble in state 2 (Figure 8.15). The interaction of these excited molecules with those at lower levels (state 1 in Figure 8.15) is described 


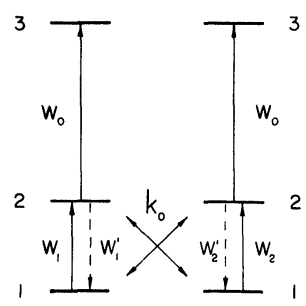

FIGURE 8.15 Model of MP dissociation selectivity loss by $V-V$ exchange. ${ }^{25}$

by one phenomenological rate constant of $V-V$ exchange $k_{v v}$. The laser excitation from state 1 to state 2 of molecules with different isotopes occurs at the rates $w_{1}$ and $w_{2}$. The rate of excitation to the energy region where molecular dissociation takes place (state 3 in Figure 8.15) will be $w_{0}$. The value of $w_{0}$ is considered to be equal for both types of molecules.

Such a model corresponds to the division of the process of molecular excitation into two steps. In the first step the excitation that can be isotopically selective takes place at several lower vibrational levels. In the second step excitation occurs in the vibrational quasicontinuum. The cross-sections of this process in most cases can be considered equal for the both isotopes due to the large spectral width of the quasi-continuum.

The equations describing the model concerned have the form

$$
\begin{aligned}
& \frac{d m_{1}}{d t}=-w_{1} m_{1}+w_{1}^{\prime} m_{2}-k_{v v} M_{2} m_{1}+k_{v v} m_{2} M_{1} \\
& \frac{d m_{2}}{d t}=-w_{0} m_{2}+w_{1} m_{1}-w_{1}^{\prime} m_{2}-k_{v v} M_{1} m_{2}+k_{v v} m_{1} M_{2} \\
& \frac{d M_{2}}{d t}=-w_{0} M_{2}+w_{2} M_{1}-w_{2}^{\prime} M_{2}-k_{v v} M_{2} m_{1}+k_{v v} m_{2} M_{1} \\
& \frac{d M_{2}}{d t}=-w_{2} M_{1}+w_{2}^{\prime} M_{1}-k_{v v} M_{1} m_{2}+k_{v v} m_{1} M_{2}
\end{aligned}
$$

where $m_{1}, m_{2}$ and $M_{1}, M_{2}$ are the populations of states 1 and 2 for molecules with the isotopes $m$ and $M$ respectively. The dissociation 
yield for each of the isotopes is determined as

$$
\beta_{1}=\frac{w_{0}}{m_{1}^{0}} \int_{0}^{\tau_{\rho}} m_{2}(t) d t \quad \beta_{2}=\frac{w_{0}}{M_{1}^{0}} \int_{0}^{\tau_{\rho}} M_{2}(t) d t
$$

where $\tau_{\rho}$ is the pulse duration, $m_{1}^{0}$ and $M_{1}^{0}$ are the initial concentrations of molecules. For the system of equations (8.9) to (8.12) to be solved analytically, ${ }^{25} m_{1}$ and $M_{1}$ are assumed to be constant which correspond to low dissociation yields $\beta_{1}, \beta_{2} \ll 1$ and the reverse transitions $w_{1}^{\prime}$ and $w_{2}^{\prime}$ are disregarded.

As a result, the selectivity of MP dissociation (mainly the molecules with the isotope $m$ are excited) can be expressed as

$$
\alpha^{\prime}=\frac{\beta_{1}}{\beta_{2}}=\frac{w_{1}\left(3-w_{0} \tau_{\rho}\right)-\left(1-x_{0}\right)\left(w_{1}-w_{2}\right) \tau_{\rho} k_{v v} \rho}{w_{2}\left(3-w_{0} \tau_{\rho}\right)+x_{0}\left(w_{1}-w_{2}\right) \tau_{\rho} k_{v v} \rho}
$$

where $p=m_{1}^{0}+M_{1}^{0}$ is the total gas pressure, and $x_{0}$ is the concentration of molecules with the isotope $m$. Expression (8.14) is derived with additional assumptions $w_{0} \tau_{\rho} \ll 1$ and $\tau_{\rho} k_{v v} \rho \ll 1$. With $p \rightarrow 0$ we get the natural result $\alpha^{\prime} \rightarrow w_{1} / w_{2} \equiv \alpha_{0}^{\prime}$, i.e., the ultimate value of selectivity is determined by the ratio of the laser excitation rates $w_{1}$ and $w_{2}$. Finally we have

$$
\alpha^{\prime}=\frac{\alpha_{0}^{\prime}\left(3-w_{0} \tau_{\rho}\right)-\left(1-x_{0}\right)\left(\alpha_{0}^{\prime}-1\right) \tau_{\rho} k_{v v} \rho}{\left(3-w_{0} \tau_{\rho}\right)+x_{0}\left(\alpha_{0}^{\prime}-1\right) \tau_{\rho} k_{v v} \rho}
$$

The slope of the dependence of $\alpha$ on $\rho$ can be expressed as

$$
v_{1}=\left.\frac{1}{\alpha_{0}^{\prime}} \frac{d \alpha^{\prime}}{d \rho}\right|_{\rho=0}=-\frac{\left(\alpha_{0}^{\prime}-1\right)\left[1+x_{0}\left(\alpha_{0}^{\prime}-1\right)\right] \tau_{\rho} k_{v v}}{\alpha_{0}^{\prime}\left(3-w_{0} \tau_{\rho}\right)}
$$

In much the same manner for excitation of molecules with the other isotope $M$ can we get

$$
\begin{gathered}
\alpha^{\prime \prime}=\frac{\beta_{2}}{\beta_{1}}=\frac{\alpha_{0}^{\prime \prime}\left(3-w_{0} \tau_{\rho}\right)-x_{o}\left(\alpha_{0}^{\prime \prime}-1\right) \tau_{\rho} k_{v v} \rho}{\left(3-w_{o} \tau_{\rho}\right)+\left(1-x_{0}\right)\left(\alpha_{0}^{\prime \prime}-1\right) \tau_{\rho} k_{v v} \rho} \\
v_{2}=\left.\frac{1}{\alpha_{0}^{\prime \prime}} \frac{d \alpha^{\prime \prime}}{d \rho}\right|_{\rho=0}=-\frac{\left(1-x_{0}\right)\left(\alpha_{0}^{\prime \prime}-1\right) \tau_{\rho} k_{v v}}{3-w_{0} \tau_{\rho}}
\end{gathered}
$$

where $\alpha_{0}^{\prime \prime}=w_{2} / w_{1}$ is the ultimate spectral value of selectivity $\alpha^{\prime \prime}$.

Comparison with experiment. The case of $\mathrm{CF}_{3} I^{25}$ The first conclusion from the solution of (8.9) to (8.12) is that the character of the depen- 
dence of selectivity on pressure may differ substantially when molecules with high and low isotope contents are excited. In the second case, with $x_{0} \ll 1$, the dependence of $\alpha^{\prime}$ on $\rho$ is governed by the value of the $\alpha_{0}^{\prime} x_{0}$ parameter. With $\alpha_{0}^{\prime} x_{0} \gg 1$ the value of selectivity depends on the partial pressure of the isotope being excited $m$ (see expression (8.15)) and with $\alpha_{0}^{\prime} x_{0} \ll 1$ on the total gas pressure. Therefore, at sufficiently high spectral selectivity the dependence of selectivity on gas pressure may be essentially slower when the poor isotope is excited. This conclusion agrees well with experiment (see Figure $8.13 a, b)$. From (8.16) and (8.17) it follows that the ratio of the rates of $\alpha$ dependence on $\rho$ for two isotopes will be

$$
\frac{v_{2}}{v_{1}}=\frac{\left(1-x_{0}\right)\left(\alpha_{0}^{\prime \prime}-1\right) \alpha_{0}^{\prime}}{\left[1+x_{0}\left(\alpha_{0}^{\prime}-1\right)\right]\left(\alpha_{0}^{\prime}-1\right)}
$$

It should be noted that $v_{2} / v_{1}$ depends only on the values of $x_{0}, \alpha_{0}^{\prime}$, $\alpha_{0}^{\prime \prime}$ and is independent of the rates of laser excitation and $V-V$ exchange. With the use of the data from Table 8.3 the value of $v_{2} / v_{1}$ can be calculated from (8.19) and compared with the experimental value. $\dagger$ With $T=-45^{\circ} \mathrm{C} v_{2} / v_{1}=62.7$ which is close to the measured value.

The rate constant of vibrational exchange $k_{v v}$ can be determined from (8.16) and (8.18) with the use of the experimental values for the slope of the dependence of $K_{\mathrm{pr}}$ on pressure, $K_{\mathrm{pr}}^{0}(13 / 12)$ and $K_{\mathrm{pr}}^{0}(12 / 13)$, pulse duration $\tau_{\rho}$. The values of $k_{v v}$ obtained for $T=+20^{\circ} \mathrm{C}$ and $T=-45^{\circ} \mathrm{C}$ are given in Table 8.3. It should be noted that the values of $K_{v v}$ obtained in independent experiments on excitation of both ${ }^{13} \mathrm{CF}_{3} \mathrm{I}$ and ${ }^{12} \mathrm{CF}_{3} \mathrm{I}$ are close. The order of magnitude for the

TABLE 8.3

Parameters of isotopically-selective dissociation of $\mathrm{CF}_{3} \mathrm{I}^{8,25}$

\begin{tabular}{lcccc}
\hline & \multicolumn{2}{c}{ Excitation of ${ }^{13} \mathrm{CF}_{3} \mathrm{I}$} & \multicolumn{2}{c}{ Excitation of ${ }^{12} \mathrm{CF}_{3} \mathrm{I}$} \\
\hline & $+20^{\circ} \mathrm{C}$ & $-45^{\circ} \mathrm{C}$ & $+20^{\circ} \mathrm{C}$ & $-45^{\circ} \mathrm{C}$ \\
\hline$K_{\mathrm{pr}}^{0}$ & 12.6 & 28 & 50 & 80 \\
$\rho_{0}$ (Torr) & $6.4 \pm 0.1$ & $10.6 \pm 0.1$ & $0.24 \pm 0.005$ & $0.2 \pm 0.005$ \\
$k_{v v}(\mathrm{~s} \cdot \text { Torr })^{-1}$ & $2.44 \cdot 10^{6}$ & $1.22 \cdot 10^{6}$ & $1.38 \cdot 10^{6}$ & $1.03 \cdot 10^{6}$ \\
\hline
\end{tabular}

$\dagger$ It was assumed that $\alpha_{0}^{\prime}=K_{\mathrm{pr}}^{0}(13 / 12)$ and $\alpha_{0}^{\prime \prime}=K_{\mathrm{pr}}^{0}(12 / 13)$. 
obtained values of $k_{v v}$ is $10^{6} \mathrm{~s}^{-1} \mathrm{Torr}^{-1}$, i.e., close to the typical value of the rate constant of $V-V$ exchange in polyatomic molecules (see Section 2.5). The dependence of $\alpha$ on $\rho$ can be calculated with formulas (8.15) and (8.17) with the use of the values of $k_{v v}$ determined above. The results of such calculation for $T=-45^{\circ} \mathrm{C}$ are shown with a dashed line in Figures 8.13a and 8.13b. The discrepancy between the experimental and calculated values with $\rho>\rho_{0}$ is caused by two factors. First, it is the simplified consideration of the processes of laser excitation and $V-V$ exchange. The variation in average level of excitation of the undesired isotope in $V-V$ exchange is not allowed for here which must affect the rate of its subsequent laser excitation. Secondly, the contribution of molecular dissociation after the laser pulse is over is not taken into account.

The model developed in Ref. 15 also correctly predicts the dependence of selectivity on laser pulse duration. The $\mathrm{CO}_{2}$ laser pulse was standard by shape with a front peak and a "tail". The pulse duration varied due to changes in the energy and duration of the "tail" as $\mathrm{N}_{2}$ was added to the working mixture of the $\mathrm{CO}_{2}$ laser. The total pulse energy was kept constant. Since the dissociation yield in this experiments with $\mathrm{CF}_{3} \mathrm{I}$ depended only on the laser pulse energy fluence and did not depend on the pulse shape, this enabled introducing the effective pulse duration

$$
\tau_{\text {eff }}=y_{0} \tau_{1}+\left(1-y_{0}\right) \tau_{2}
$$

where $\tau_{1}$ and $\tau_{2}$ are the widths (at half height) of the pulse front peak and "tail", $y_{0}$ is the fraction of total pulse energy contained in the front peak. In the experiment two types of pulses were used, short $\tau_{s}$ and long $T_{l}$. Their effective duration was $\tau_{s}=1.85 \cdot 10^{-7} \mathrm{~s}, \tau_{l}=$ $7.75 \cdot 10^{-7} \mathrm{~s}$.

From expression (8.15) and (8.17) it is possible to estimate the values of $\alpha^{\prime}$ and $\alpha^{\prime \prime}$ for different pulse durations $\tau_{s}$ and $\tau_{l}$ on the assumption that the ultimate spectral selectivity $\left(\alpha_{0}^{\prime}\right.$ and $\left.\alpha_{0}^{\prime \prime}\right)$ does not depend on $\tau_{\rho}$. This assumption is quite reasonable. The experiments on $\mathrm{SF}_{6},{ }^{17}$ for example, carried out at a low gas pressure $1.6 \cdot 10^{-2}$ Torr, when the collisions may be neglected, have shown that selectivity is independent of laser pulse duration. In the estimates the values of $k_{v v}$ presented in Table 8.3 were used. The results obtained for exciting both ${ }^{13} \mathrm{CF}_{3} \mathrm{I}$ and ${ }^{12} \mathrm{CF}_{3} \mathrm{I}$ are given in Table 8.4, where the experimentally measured values of $K_{\mathrm{pr}}(13 / 12)$ and $k_{\mathrm{pr}}(12 / 13)$ can be found, too. The com- 
TABLE 8.4

Dependence of $\mathrm{CF}_{3} \mathrm{I}$ dissociation selectivity on laser pulse duration ${ }^{25}$

\begin{tabular}{lcccc}
\hline $\begin{array}{l}\text { Excitation of }{ }^{13} \mathrm{CF}_{3} \mathrm{I} \\
\begin{array}{l}p=0.8 \text { Torr } \\
T=-45^{\circ} \mathrm{C}\end{array}\end{array}$ & & \multicolumn{3}{c}{$\begin{array}{c}\text { Excitation of }{ }^{12} \mathrm{CF}_{3} \mathrm{I} \\
p=0.2 \text { Torr } \\
T=+20^{\circ} \mathrm{C}\end{array}$} \\
\hline & Experiment & Model & Experiment & Model \\
\hline Short pulse & 25.2 & 25.9 & 21.8 & 27.3 \\
Long pulse & 17.9 & 19.8 & 10.2 & 11.2 \\
Ratio (short/long) & 1.41 & 1.31 & 2.14 & 2.44 \\
\hline
\end{tabular}

parison of the results of experiment and calculations shows that the model used agrees well with experiment.

These experiments with different pulse durations demonstrate that $V-V$ exchange during a laser pulse is the main channel of selectivity loss in $\mathrm{CF}_{3} \mathrm{I}$. At the same time these measurements show that, at least under the conditions of experiments with $\mathrm{CF}_{3} \mathrm{I}$, exchange reactions like (8.23) cannot be the main contribution to spectral selectivity loss because the characteristic time of such reactions is several orders longer than the laser pulse duration.

\section{Possibilities of decrease in the role of $V-V$ exchange}

The comparison of the model with experiment shows that, even though the model is simplified in character, it correctly describes the action of $V-V$ exchange during an excitation pulse on the isotopic selectivity of MP dissociation. So the conclusions of this model can be used to analyze the possibilities of a decrease in the effect of $V-V$ exchange and an increase in the primary dissociation selectivity.

It follows from the model that in exciting a component with a low content of desired isotope $\left(x_{0} \ll 1\right)$ it is possible to work with a high total gas pressure if the spectral dissociation selectivity $\alpha_{0}$ is high. The criterion of fulfilling this condition is $\alpha_{0} \geqslant 1 / x_{0}$. In this case the enrichment factor depends on the partial pressure of the desired isotope and so the gas pressure may be sufficiently high. This conclusion has been supported by experiments. In the experiments on $\mathrm{CF}_{3} \mathrm{I}^{8,25}$ the condition $\alpha_{0} \simeq 1 / x_{0}$ was fulfilled and so high dissociation selectivity of ${ }^{13} \mathrm{CF}_{3} \mathrm{I}$ was observed at a high total pressure of $\mathrm{CF}_{3} \mathrm{I}$. In experiments $/ 8.28 /$ with $\mathrm{SF}_{6}$ the value of $\alpha_{0} x_{0}$ was small $\left(\alpha_{0} x_{0}=0.06 \ll\right.$ 1) due to insufficiently high spectral selectivity of ${ }^{33} \mathrm{SF}_{6}$ dissociation $\left(\alpha_{0} \simeq 7\right)$. This factor is apparently responsible for the fast dependence 
of $\alpha$ on $\rho$ observed in the experiment, approximately as in exciting ${ }^{32} \mathrm{SF}_{6}$.

Thus, an increase in spectral excitation selectivity is one of the ways of decreasing the effect of the process of $V-V$ exchange as molecules with a low content of desired isotope dissociate.

One more way of increasing the primary dissociation selectivity consists of reducing the exciting pulse duration. In this case, when the process of $V-V$ exchange during a laser pulse has a dominant role in decreasing the selectivity, a decrease in $\tau_{\rho}$ causes, of course, the contribution of $V-V$ exchange to the measured value of selectivity to fall. This conclusion was confirmed in experiments on $\mathrm{CF}_{3} \mathrm{I}$ where it was demonstrated that a reduction in pulse duration brought about an increase of $\alpha$ in exciting molecules both with the poor and rich isotope ${ }^{13} \mathrm{CF}_{3} \mathrm{I}$ and ${ }^{12} \mathrm{CF}_{3} \mathrm{I}$. Therefore, by decreasing the laser pulse duration one can increase the total gas pressure with the dissociation selectivity remaining high. A striking proof of this is the work on hydrogen isotope separation. ${ }^{21}$ Having reduced the laser pulse duration to $2 \times 10^{-9} \mathrm{~s}$ a mixture of $\mathrm{CF}_{3} \mathrm{D}$ and $\mathrm{CF}_{3} \mathrm{H}$ could yield an enrichment factor in the products $K_{\mathrm{pr}}(D / H)=11000$ at a total gas pressure of 100 Torr.

It follows from the above model that during resonant excitation of molecules with a rich isotope the condition

$$
\rho_{0} \alpha_{0}^{\prime \prime} k_{v v} \tau_{\rho} \simeq \text { const }
$$

is fulfilled. This expression, in particular, shows why at a comparatively low rate of $V-V$ exchange $k_{v v} \sim 10^{6} \mathrm{~s}^{-1} \mathrm{Torr}^{-1}$ the selectivity can be decreased considerably at still small values $\rho \tau_{\rho}<1 / k_{v v}$. The point is that the whole rate of $V-V$ exchange between two systems (in our case between molecules with different isotopes) is determined not only by the value of $k_{v v}$ but is also proportional to the degree of deviation of these systems from equilibrium with each other, i.e., the value of excitation selectivity. This is responsible for the appearance of $\alpha_{0}$ in expression (8.21). (As molecules with a poor isotope are excited, if $\alpha_{0} x_{0} \gg 1$, expression (8.21) has the same form, but $\rho_{0}$ here denotes the partial pressure of the isotope under excitation.)

From (8.21) it follows that by changing the spectral selectivity, for example through gas temperature or frequency tuning of exciting radiation, one can affect the rate of $V-V$ exchange and hence vary the rate of the dependence of $\alpha$ on $\rho$. It is this factor that decelerates 
the dependence of $K_{\mathrm{pr}}(12 / 13)$ on the pressure of $\mathrm{CF}_{3} \mathrm{I}$ (Figure 8.13b) as the gas temperature rises. Expression (8.21) makes it possible to optimize the irradiation frequency, gas temperature and pressure in isotopically selective MP dissociation. In some cases we can profitably decrease the spectral selectivity to increase the gas pressure at some required fixed value $\alpha^{\prime \prime}<\alpha_{0}^{\prime \prime}$.

$V-V$ exchange after the excitation pulse. At this stage $V-V$ exchange can also affect the parameters of MP dissociation and particularly its selectivity. In the first experiments on MP dissociation of molecules ${ }^{1,2}$ it was shown that the redistribution of vibrational energy as a recult of $V-V$ exchange can contribute considerably to the observed dissociation yield forming its so-called collisional phase. The investigation of the isotopic selectivity of this dissociation stage ${ }^{54}$ was actually the first experiment on the influence of collisions on the selectivity of MP dissociation. The experiment was performed with ${ }^{10} \mathrm{BCl}_{3}$ and ${ }^{11} \mathrm{BCl}_{3}$ and its aim was to study the selectivity of formation of the dissociation fragments of these molecules at different instants of time after the $\mathrm{CO}_{2}$ laser pulse. The results of this work show that selectivity is preserved in the collisional dissociation phase, too, at least during (1-2) $\tau_{v v}$. Later the selectivity of collisional dissociation phase was studied theoretically. ${ }^{55}$ The model calculations carried out show that selectivity may actually take place in this dissociation phase during $(0-2) \tau_{v v}$ even though it is reduced in time in this case. The selectivity value here is determined by the difference in excitation level of the two components and their relative concentrations.

The cases considered above are basically concerned with exciting rather few-atomic molecules. As it follows from the RRKM theory (see Section 2.4), these molecules dissociate in $10^{-9}$ to $10^{-7} \mathrm{~s}$ even at low excitation over the dissociation limit ( 1 or 2 quanta of $\mathrm{CO}_{2}$ laser). A different situation exists for bigger molecules consisting of eight, nine or more atoms. For these molecules the time of unimolecular decay is increased by 2 or 3 orders and even more. The laser pulse duration being $\sim 10^{-7} \mathrm{~s}$, the dissociation of such molecules would occur mainly when the pulse is over. In this situation the $V-V$ exchange with cold molecules may cause deexcitation of vibrationally excited molecules and, accordingly, a decrease in dissociation yield. The deexcitation role of $V-V$ exchange would apparently show itself most efficiently when the laser excites the poor component. The dependence of the dissociation yield on the pressure of $\mathrm{CF}_{3} \mathrm{COCF}_{3}{ }^{51}$ from exciting 
$\mathrm{CF}_{3}{ }^{13} \mathrm{COCF}_{3}$ is characteristic in this sense (see Figure 8.14). The increase of the gas pressure from 0.2 Torr to 10 Torr results in a decrease in yield by about three orders.

It would be of interest to explain the observed increase in selectivity due to a different contribution of the process of deexcitation to the dissociation yield of different components. Indeed, as the research shows, the dissociation yield is determined mainly by the energy stored in a molecule, i.e., it is a function of the average level of molecular excitation $\beta=f(\bar{\varepsilon})$. This dependence increases with a decrease in $\bar{\varepsilon}$. In exciting the poor component the average energy level of excited molecules after the pulse is over must exceed the corresponding value for the rich component if the selectivity is sufficiently high. According to the above, the decrease in $\bar{\varepsilon}$ from $V-V$ exchange with the ensemble of cold molecules must affect more the dissociation yield of the molecules of the rich component. As a result, it must increase the selectivity of MP dissociation as the pressure rises.

It is not impossible that it is just such a mechanism that takes place for $\mathrm{CF}_{4} \mathrm{COCF}_{3}$. The data available, however, is not sufficient for a final conclusion especially as an analogous increase in selectivity was observed in the experiments with $\mathrm{CF}_{3} \mathrm{Br}$ both with increasing gas pressure $/ 8.52,8.53 /$ and with a buffer added.$^{53}$ In these experiments the effect of $V-V$ exchange after the laser pulse can be neglected.

It is the dependence of the function $\alpha=\alpha(\rho)$ on the excitation radiation frequency ${ }^{53}$ that can apparently give a key to understanding the causes of such an increase in $\alpha$. The contribution of rotational relaxation is one of the probable causes of such a dependence. The potential influence of rotational relaxation on the isotopic selectivity of MP dissociation will be considered below.

\subsubsection{Collisions with buffer gas. Effect of $V-T$ and rotational relaxation}

In parallel with $V-V$ exchange, the processes of $V-T$ and rotational relaxation also take place as a result of collisions. $\dagger$ All the necessary information on these processes is given in Section 2.5. We want to recall that $V-T$ relaxation is related to the transfer of a part of vibrational energy to translational degrees of freedom. This, in

\footnotetext{
† Collisions also induce phase relaxation but the effect of this process on the isotopic selectivity of MP dissociation has not yet been revealed, and so will not be considered.
} 
particular, leads to vibrational deexcitation of excited molecules especially when they are highly diluted with cold molecules.

Rotational relaxation causes an equilibrium distribution over rotational levels to be reached after a perturbation. Besides a great difference in rate constants, the processes of $V-T$ and rotational relaxation also differ by the fact that the establishment of rotational equilibrium for polyatomic molecules does not significantly change their vibrational energy.

The action of rotational and $V-T$ relaxation is rather often masked by the high-rate resonant $V-V$ exchange, and so the effect of these processes on the isotopic selectivity of MP dissociation can be studied more conveniently with the use of buffer gases. Usually for these purposes one- or two-atom gases are used. This makes it possible to eliminate the effect of $V-V$ exchange and thereby reveal the role of the two other relaxation processes.

After these preliminary notes we are going to proceed to considering the available data.

Experimental results. There have been rather few experiments dealing with the effect of the addition of different gases on selectivity. For example, it has been shown ${ }^{14}$ that the addition of 0.5 Torr $\mathrm{NH}_{3}$ to 0.2 Torr $\mathrm{SF}_{6}$ increases $K_{\mathrm{pr}}(32 / 24)$ by one and a half times. The addition of 20 Torr oxygen had the same effect. Such behavior of $K_{\mathrm{pr}}$ was explained ${ }^{14}$ by a general decrease in the average energy level of molecules due to their deexcitation by the buffer gas.

An increase in dissociation selectivity with increasing pressure of $\mathrm{N}_{2}+\mathrm{O}_{2}$ has been also observed in experiments with $\mathrm{BCl}_{3}{ }^{9}{ }^{9}$ It must be noted, however, that in this case $\mathrm{O}_{2}$ also acts as an acceptor and so the measured increase of $K_{\mathrm{pr}}$ may be also related to the suppression of nonselective chemical reactions (see below). Some increase in dissociation selectivity was observed during addition of buffer gas in the experiments with $\mathrm{CF}_{2} \mathrm{Cl}_{2}{ }^{56}$ and $\mathrm{CF}_{3} \mathrm{I}^{8}$.

In experiments on $\mathrm{SF}_{5} \mathrm{Cl}^{57}$ the addition of hydrogen that acted as an acceptor at the same time also brought about a small increase in the value of $\alpha(32 / 34)$. The addition of nitrogen to 0.25 Torr $\mathrm{SF}_{5} \mathrm{Cl}$ reduced the dissociation selectivity from $\alpha(32 / 34)=2.33$ with $\rho_{\mathrm{N}_{2}}=0$ to 1.33 with $\rho_{\mathrm{N}_{2}}=3$ Torr. A decrease in selectivity with increasing pressure of $\mathrm{H}_{2}$ has been also observed in experiments with $\mathrm{SF}_{6} .{ }^{35}$

More detailed studies have been carried out. ${ }^{58}$ In these experiments the total dissociation of ${ }^{32} \mathrm{SF}_{6}$ and ${ }^{34} \mathrm{SF}_{6}$ was measured in a cell during 
resonant excitation of ${ }^{32} \mathrm{SF}_{6}$ in the presence of different buffer gases by a $\mathrm{CO}_{2}$ laser. Some results of these measurements are discussed below.

It is impossible to find the value of $\alpha$ from the data ${ }^{58}$ with the use of expression (8.4) because of the high total dissociation of $\mathrm{SF}_{6}$. Nevertheless, this data shows the general qualitative picture of buffer gas influence on selectivity. As small amounts of such monoatomic gases as $\mathrm{Ar}, \mathrm{Ne}$ and $\mathrm{He}$ were added, the dissociation of ${ }^{32} \mathrm{SF}_{6}$ almost did not change. At the same time there is an increase in the dissociation of ${ }^{34} \mathrm{SF}_{6}$ which corresponds to a decrease in selectivity of the process of dissociation. With strong dilution of $\mathrm{SF}_{6}$ with a buffer $(1: 100)$ the dissociation of ${ }^{32} \mathrm{SF}_{6}$ is reduced considerably, the dissociation of ${ }^{34} \mathrm{SF}_{6}$ changing somewhat less, which corresponds to a further decrease in selectivity as compared to the case of pure $\mathrm{SF}_{6}$.

The results are explained ${ }^{58}$ by $V-T$ and rotational relaxation. With addition of small amounts of a buffer the main contribution is by rotational relaxation discussed in more detail below.

With larger addition of buffer gases the variation of $\alpha$ is related to the deexcitation of the molecules excited by the laser radiation as a result of $V-T$ relaxation. As follows from the data, ${ }^{58}$ the lighter the buffer, the higher the deexcitation, which is very characteristic of the $V-T$ process. Nevertheless, it is not impossible that at such pressures, as well, rotational relaxation makes its own contribution.

The results presented show that the effect of buffer gases on selectivity is caused by different processes and so its character may depend on such factors as the type of buffer and its pressure as well as the conditions of laser excitation. The measurements with $\mathrm{H}_{2}$ directly point to this. The experiments show that with addition of hydrogen the selectivity increases unlike the case of monoatomic gases and the measurements with $\mathrm{SF}_{6}{ }^{35}$ already mentioned above.

Thus, the results presented are contradictory at first glance. In some experiments the addition of a buffer increases the selectivity, in other ones it decreases the selectivity. Other studies ${ }^{53}$ make things somewhat clearer, and the results will be considered in more detail.

In these experiments ${ }^{53}$ consideration was given to the effect of gas (buffer and parent) pressure on the MP dissociation selectivity of ${ }^{13} \mathrm{CF}_{3} \mathrm{Br}$ under different conditions of excitation. The $\mathrm{CF}_{3} \mathrm{Br}$ were excited at the $P(12)$ and $P(32)$ lines of the $\mathrm{CO}_{2}$ laser in the $9.6 \mu$ 
region. This corresponds to the excitation of ${ }^{13} \mathrm{CF}_{3} \mathrm{Br}$ approximately at the center of the $\nu_{1}$ band $\left(\nu_{1}-52(P(12))=3.7 \mathrm{~cm}^{-1}\right)$ and at its long-wave end $\left(\nu_{1}-52(P(32))=22.2 \mathrm{~cm}^{-1}\right)$. The detunings from the band of ${ }^{12} \mathrm{CF}_{3} \mathrm{Br}$ were respectively $30 \mathrm{~cm}^{-1}$ and $48.5 \mathrm{~cm}^{-1}$. The values of $\alpha(13 / 12)$ measured at different pressures of the buffer gas $\mathrm{N}_{2}$ are given in Figure 8.16a. It may be seen that the behavior of the function $\alpha=\alpha\left(\rho_{\mathrm{N}_{2}}\right)$ changes radically with radiation frequency. As the excitation frequency is moved towards the center of the band of ${ }^{13} \mathrm{CF}_{3} \mathrm{Br}$ the selectivity decreases with addition of nitrogen (curve 1) and at the edge of the band it increases (curve 2).

With $\rho<10$ Torr the dependence of $\alpha$ on the pressure of $\mathrm{CF}_{3} \mathrm{Br}$ has the same qualitative character (curves 3 and 4). The difference lies in a faster pressure dependence. The variation in gas temperature also affects the form of the function $\alpha=\alpha(\rho)$ (compare curves 5 and 4).

The results presented as well as the measurements of the dissociation yield for ${ }^{13} \mathrm{CF}_{3} \mathrm{Br}$ and ${ }^{12} \mathrm{CF}_{3} \mathrm{Br}$ leads to the conclusion that it is mainly rotational relaxation that is responsible for such behavior of $\alpha$. The mechanism of its action will be clear from the model below.

Rotational relaxation model. ${ }^{53}$ Let us consider the case of exciting two isotopic components of $N_{i}(i=1,2)$ in the presence of the buffer gas $M$. In the previous chapters it has been shown that molecules at different rotational levels can interact with the laser radiation in different ways. For simplicity let us assume that the field interacts only with a certain group of levels the equilibrium population of which $n_{i}^{e}=f_{i} N_{i}$. The excitation from these levels comes about at the rate $w_{i}$, and as a result of rotational relaxation they are repopulated with the rate constant $k_{r}$. In this case the following relations

$$
\begin{aligned}
& \dot{n}_{i}=-w_{i} n_{i}+k_{r} M\left(f_{i} N_{i}-n_{i}\right) \\
& \dot{N}_{i}=-w_{i} N_{i}
\end{aligned}
$$

are fulfilled. These equations describe the effect of rotational relaxation in the ground state. A similar approach has been applied with success to describe the effect of a rotational "bottle neck" at single-photon excitation (see Section 3.3). To describe multiple photon excitation, equations (8.22) can be supplemented with an appropriate system of equations describing the subsequent excitation in the quasi-continuum (see Chapter 6). But in our case this is not necessary, and we can use for analysis the reduced system (8.22). This is due to the fact that, as 
was shown in experiments on $\mathrm{SF}_{6}$ and $\mathrm{CF}_{3} \mathrm{I}$ (see Chapter 6), the action of rotational relaxation is limited basically to populating the depleted rotational levels in the ground state and it affects only slightly the subsequent excitation of molecules, at least in these two molecules.

The solution of equation (8.22) shows that the value of the contribution of rotational relaxation to dissociation selectivity as well as its sign are governed by the relations between $w_{i} \tau_{\rho}, f_{i}$ and $k_{r} M \tau_{\rho}$.

Let both components interact effectively with the field, i.e., $w_{i} \tau_{\rho} \gg 1$, $i=1,2$. This corresponds to the saturation of resonant levels and the formation of deep "holes" in the rotational distribution. It can be shown that in this case $\alpha$ always decreases with addition of a buffer gas.

With only the desired component saturated, $\alpha$ can be increased with increasing pressure. We shall not write out the qualitative relations between $w_{i} \tau_{\rho}, f_{i}$ and $k_{r} M \tau_{\rho}$ at which an increase in $\alpha$ occurs. These cases are comprehensively discussed in Ref. 53 . The general qualitative conclusion is that an increase in $\alpha$ takes place when the relative contribution of rotational relaxation to the MP dissociation yield for the desired component is larger than that for the undesired one. This evidently occurs when the degree of rotational distribution disturbance from equilibrium caused by radiation is smaller for the undesired component. This, for example, can be when the "hole" in the rotational distribution of this component is small. In this case we may even have $f_{2}=1$, i.e., all the molecules interact with the field, although slightly $\left(w_{2} \tau_{\rho} \ll 1\right)$. Rotational relaxation in this case does not contribute to $\beta_{2}$ as it increases $\beta_{1}$, and hence $\alpha=\beta_{1} / \beta_{2}$ if $f_{1}<1$.

Now let $f_{1}=1$ and $w_{1} \tau_{\rho} \gg 1$, i.e., conversely all the molecules of the desired component effectively interact with the field. In this case rotational relaxation contributes only to the value of $\beta$ (if $f_{2}<1$ ) and hence decreases $\alpha$. This case apparently took place with $\mathrm{SF}_{6}{ }^{58}$ since with the energy fluence used all the molecules of the desired component, i.e., ${ }^{32} \mathrm{SF}_{6}$, interacted with the field.

Discussion of results. Turning back to the experiments with $\mathrm{CF}_{3} \mathrm{Br}$ we should note that the measurements of the MP dissociation yield provide us with a powerful argument in favor of the discussed mechanism of the effect of rotational relaxation. With an addition of a buffer gas one could always observe an increase in $\beta_{1}$ and $\beta_{2}$. The character of the dependence may be seen from Figure $8.16 \mathrm{~b}$ which presents the result for $\beta_{13}$ during excitation at the band edge (curve 1). Similar 


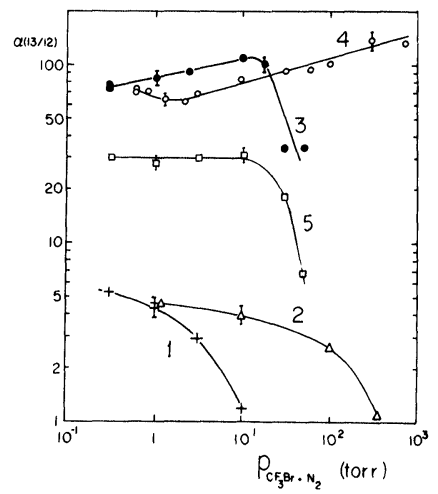

FIGURE 8.16a MP dissociation selectivity $\alpha(13 / 12)$ of $\mathrm{CF}_{3} \mathrm{Br}$ molecules as a function of gas pressure. $(1,2)$ the pressure of $\mathrm{N}$ varies with $\rho_{\mathrm{CF}_{3} \mathrm{Br}}=0.3 \mathrm{Torr} ;(3,4,5)$ the pressure of $\mathrm{CF}_{3} \mathrm{Br}$ varies with $\rho_{\mathrm{N}_{2}}=0 ;(5)$ gas temperature $T=356 \mathrm{~K}$, the rest of the curves are plotted for $T=293 \mathrm{~K}$. $(1,3)$ excitation at the $P(12)$ line; $(2,4,5)$ excitation at the $P(32)$ line. The value of $\Phi$ everywhere is $4 \mathrm{~J} / \mathrm{cm}^{2} .53$

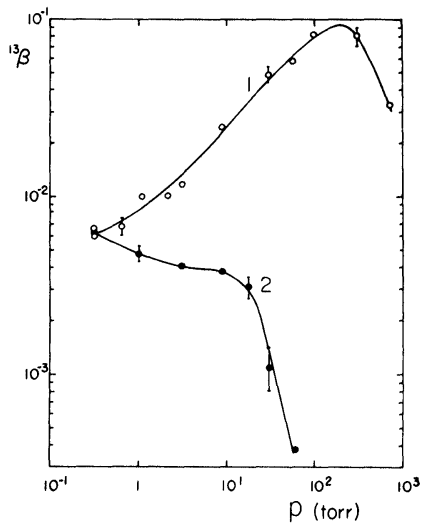

FIGURE 8.16b Dependence of the MP dissociation yield of ${ }^{13} \mathrm{CF}_{3} \mathrm{Br}$ on the pressure of $\mathrm{N}_{2}$ (curve 1) and $\mathrm{CF}_{3} \mathrm{Br}$ (curve 2) with excitation at the $P(32)$ line. ${ }^{53}$

dependences can be observed for quite a number of molecules (see Section 7.4.1). They all are caused by the action of rotational relaxation. The contribution of $V-T$ relaxation manifests itself much later. In experiments with $\mathrm{CF}_{3} \mathrm{Br}$ the deexcitation caused by this process becomes essential with $\rho_{\mathrm{N}_{2}}>100$ Torr. Thus, at lower pressures the 
only collisional process that affects the MP dissociation of both components is rotational relaxation.

Another argument in favor of this mechanism is the possibility of changing the form of the dependence $\alpha=\alpha(\rho)$ by changing only the radiation frequency. Such a change apparently brings about a change in the fraction of molecules $f_{i}$ of both components interacting with the field. As follows from the above model, the ratio between the values of $f_{i}$ and $w_{i} \tau_{\rho}$ is a critical factor which determines the contribution of rotational relaxation and its sign.

The dependence of $\alpha$ on the pressure of $\mathrm{CF}_{3} \mathrm{Br}$ in Figure 8.16a is also apparently caused by the similar action of rotational relaxation but the situation is more complicated. As it may be seen from Figure $8.16 \mathrm{~b}, V-V$ exchange makes a considerable contribution to the process of MP dissociation. The difference in character of curves 1 and 2 is caused by the different mechanisms of deexcitation. $V-V$ exchange with cold ${ }^{12} \mathrm{CF}_{3} \mathrm{Br}$ molecules is the mechanism in the second case.

The rate constants of the $V-V$ (the parent gas) and $V-T$ (the buffer) processes usually differ by 2 or 3 orders. This is responsible for the difference by 2 orders between the rate of decrease of $\beta_{13}$ with $\rho$ for curves 1 and 2. With $\rho_{\mathrm{CF}_{3} \mathrm{Br}}<10$ Torr (curve 2) rotational relaxation can only partially compensate for the decrease of $\beta_{13}$ as a result of $V-V$ transfer. At a higher pressure the $V-V$ exchange becomes a dominating factor, and it is responsible for a drastic decrease of $\alpha$ with $\rho_{\mathrm{CF}_{3} \mathrm{Br}}>10$ Torr. It is not impossible that it contributes to selectivity at lower pressures as well. The competition between laser excitation and the deexcitation due to the process of $V-V$ exchange, for example, was considered ${ }^{52}$ to be responsible for the increase of $\alpha$ with increasing $\rho$ during excitation at the edge of the ${ }^{13} \mathrm{CF}_{3} \mathrm{Br}$ band.

Finally, Figure 8.16a shows that a variation in gas temperature also affects the character of the dependence $\alpha=\alpha(\rho)$. This seems to be related to the change of the values of $f_{i}$ resulting from the variation of initial molecular distribution. However, the factors considered in Section 8.3 can also produce an effect here.

In conclusion we should stress again that the processes of $V-T$ and rotational relaxation act on selectivity only when they make a different contribution to the MP dissociation yield of two isotopic components. As far as rotational relaxation is concerned, its action on selectivity as well as the sign of this action depend on the conditions of excitation. 
One of the ways of increasing selectivity due to rotational relaxation is detuning from the center of the excitation band of both components. In this case we can set the conditions when rotational relaxation makes a larger contribution to the dissociation yield of the desired component. The increase in the number of molecules involved in the process of MP excitation caused by rotational relaxation $\mathrm{can}^{53}$ considerably compensate for the decrease in dissociation yield of the desired component caused by the detuning from the center of the band.

\subsection{Loss of MP dissociation selectivity in secondary chemical processes}

At the end of this chapter we want to consider one more channel of selectivity loss. This may be chemical reactions in which the fragments resulting from molecular dissociation participate. In a number of intermediate reactions the primary dissociation selectivity may be lost to a greater or lesser degree. There are a large number of possibilities of reaction types here but the final result of all of them is that the molecules of the initial substance are involved nonselectively in chemical reactions. They can react with both intermediate and end products of reactions of dissociation fragments. The last possibility has been given particular emphasis. ${ }^{34}$ In this work the authors studied, among other things, the dependence of $\alpha$ on the number of radiation pulses during dissociation of $\mathrm{BCl}_{3}$ in mixture with $\mathrm{H}_{2}$. The experiments show that the value of $\alpha$ decreases as the gas is irradiated. The authors ${ }^{34}$ relate this decrease to the accumulation of new products in the cell and their reaction with the original $\mathrm{BCl}_{3}$ molecules.

The exchange reaction with an atom or a radical group can be considered as one more channel of primary selectivity loss. This can be illustrated by such a reaction

$$
{ }^{i} A+{ }^{k} A B \rightleftarrows{ }^{i} A B+{ }^{k} A
$$

The exchange with the iodine atom by scheme (8.23) during dissociation of $\mathrm{CF}_{3} \mathrm{I}$ (the molecule dissociates into $\mathrm{CF}_{3}$ and $\mathrm{I}$ ) has even been considered $^{59}$ as the main channel of selectivity loss. This conclusion, as applied to $\mathrm{CF}_{3} \mathrm{I}$, however, cannot be apparently justified (see Section 8.5.2). In general such reactions, however, can occur and result in a decrease in primary selectivity.

It is important to choose a proper scavenger for binding the resultant dissociation fragments as illustrated ${ }^{9}$ with experiments on $\mathrm{BCl}_{3}$. As it 
has been already said, the dissociation of $\mathrm{BCl}_{3}$ is reversible and thus scavengers should be used. In the experiments $\mathrm{HBr}, \mathrm{O}_{2}, \mathrm{C}_{2} \mathrm{D}_{2}, \mathrm{NO}$ and $\mathrm{H}_{2}$ were used as acceptors. It follows from these experiments that the value of $K_{\mathrm{pr}}(10 / 11)$ measured from the final results of irradiation essentially depends on the kind of scavenger and hence on the type of intermediate reactions. In case of $\mathrm{HBr}$, for example, the value $K_{\mathrm{pr}}$ is two times higher than in case of $\mathrm{H}_{2}$.

When $\mathrm{O}_{2}$ is used as a scavenger, the selectivity may be measured at early stages of the reaction. In Ref. $54 K_{\mathrm{pr}}$ was measured at the first stage, the formation of the $\mathrm{BO}$ radical, from the chemiluminescence spectrum. $\dagger$ At this stage $K_{\mathrm{pr}} \simeq 10$ which was much higher than the value obtained from the end products of irradiation. This result obviously points to the fact that the decrease in selectivity occurred in the process of chemical formation of end products.

A decrease in selectivity due to secondary chemical reactions was also observed in experiments with pentafluorobenzene. ${ }^{60}$ In isotopically selective dissociation of $\mathrm{C}_{6} \mathrm{~F}_{5} \mathrm{H}$ and $\mathrm{C}_{6} \mathrm{~F}_{5} \mathrm{D}$ it was found that the atoms and radicals resulting in the dissociation of these molecules reacted with the original substance and thereby decreased the final selectivity of the entire process. By adding different scavengers it was possible ${ }^{61}$ to reduce the contribution of these reactions and, as a result, to increase the measured value of $\alpha$.

An essential role of secondary chemical processes was disclosed in MP dissociation of trans- $\mathrm{ClHC}=\mathrm{CHBCl}_{2}$ molecules. ${ }^{62}$ It was found that the quantum yield of dissociation was higher than unity. This allowed drawing a conclusion of a great contribution of secondary chain chemical reactions. These reactions reduce the primary selectivity of MP dissociation (for boron isotopes) and give rise to comparatively low values of $\alpha$ in end products.

In conclusion it should be mentioned the possibility of chemical reactions for vibrationally-excited molecules. It has been so far believed that the formation of enriched end products in the presence of scavenger takes place only when the fragments of molecular dissociation react. However, scavengers can react with highly vibrationallyexcited molecules too. Such reactions, in principle, can occur and make their contribution to the observed value of selectivity.

$\dagger$ While studying the isotopic effects in the spectra of resulting radicals, it is possible in principle, to follow the value of $\alpha$ at all the stages of the reaction. 


\section{References}

1. N. R. Isenor, V. Merchant, R. S. Hallsworth and M. C. Richardson, Canad.J.Phys. 51, 1281 (1973).

2. R. V. Ambartzumian, N. V. Chekalin, V. S. Doljikov, V. S. Letokhov and E. A. Ryabov, Chem. Phys. Lett. 25, 515 (1974).

3. R. V. Ambartzumian, V. S. Letokhov, E. A. Ryabov and N. V. Chekalin, ZhETF Pis'ma Red. 20, 597 (1974) [Sov. Phys. JETP Lett. 20, 273 (1974)].

4. J. A. Horsley, D. M. Cox, R. B. Hall, A. Kaldor, E. T. Maas, Jr., E. B. Priestley and G. M. Kramer, J. Chem. Phys. 73, 3660 (1980).

5. E. P. Velikhov, V. S. Letokhov, A. A. Makarov and E. A. Ryabov, Kvantovaya Electron. (Russian) 6, 317 (1979).

6. N. V. Chekalin, V. S. Doljikov, Yu. R. Kolomisky, V. S. Letokhov, V. N. Lokhman and E. A. Ryabov, Appl. Phys. 13, 311 (1977).

7. R. V. Ambartzumian, Yu. A. Gorokhov, V. S. Letokhov and G. N. Makarov, ZhETF Pis'ma Red. 21, 375 (1975) [Sov. Phys. JETP Lett. 21, 171 (1975)].

8. V. N. Bagratashvili, V. S. Doljikov, V. S. Letokhov and E. A. Ryabov, Pis'ma Zh. Techn. Fiz. (Russian) 4, 1181 (1978).

9. R. V. Ambartzumian, Yu. A. Gorokhov, V. S. Letokhov, G. N. Makarov, E. A. Ryabov and N. V. Chekalin, Kvantovaya Electron. 2, 2197 (1975) [Sov. J. Quantum. Electron. 5, 1196 (1975)].

10. F. Brunner, T. P. Cotter, K. L. Kompa and D. Proch, J. Chem. Phys. 67, 1547 (1977).

11. M. J. Coggida, P. A. Schulz, Y. T. Lee and Y. R. Shen, Phys. Rev. Lett. 38, 17 (1977).

12. J. D. Campbell, G. Hancock, J. B. Halpern and K. N. Welge, Optics Commun. 17, 38 (1976).

13. N. V. Chekalin, V. S. Doljikov, V. S. Letokhov, V. N. Lokhman and A. N. Shibanov, Appl. Phys. 12, 191 (1977).

14. R. V. Ambartzumian, Yu. A. Gorokhov, V. S. Letokhov, G. N. Makarov and A. A. Puretzkii, Zh. Eksp. Teor. Fiz. (Russian) 71, 440 (1976).

15. R. V. Ambartzumian and V. S. Letokhov, in: Chemical and Biochemical Applications of Laser, Vol. III, ed. C. B. Moore (Academic Press, New York, San Francisco, London, 1977) p. 167.

16. Yu. R. Kolomiiskii and E. A. Ryabov, Kvantovaya Electron. (Russian) 5, 651 (1978).

17. M. C. Gower and K. W. Billman, Optics Commun. 20, 123 (1977).

18. R. V. Ambartzumian, Yu. A. Gorokhov, V. S. Letokhov, G. N. Makarov and A. A. Puretzkii, ZhETF Pis'ma Red. 22, 374 (1975) [Sov. Phys. JETP Lett. 22, 177 (1975)].

19. S. F. Freund and J. L. Lyman, Chem. Phys. Lett. 55, 435 (1978).

20. V. N. Bagratashvili, V. S. Doljikov, V. S. Letokhov and E. A. Ryabov in: Laser Induced Processes in Molecules, Springer Series in Chemical Physics, Vol. 6, eds K. L. Kompa and S. D. Smith, Berlin, Heidelberg, New York, 1979) p. 179.

21. J. B. Marling, I. P. Herman and S. J. Thomas, J. Chem. Phys. 72, 5603 (1980).

22. R. V. Ambartzumian, V. M. Apatin, A. V. Evseev and N. P. Furzikov, Kvantovaya Electron. (Russian) 7, 1998 (1980).

23. P. Fettweis and M. Neve de Mevergnies, J. Appl. Phys. 49, 5699 (1978).

24. M. Meve de Mevergnies and P. del Marmol, J. Chem. Phys. 73, 3011 (1980).

25. V. N. Bagratashvili, V. S. Doljikov, V. S. Letokhov and E. A. Ryabov, Appl. Phys. 20, 231 (1979).

26. T. F. Deutsch, Optics Lett. 1, 25 (1977).

27. R. V. Ambartzumian, Yu. A. Gorokhov, V. S. Letokhov and G. N. Makarov, $Z h$. Eksp. Teor. Fiz. 69, 1956 (1975) [Sov. Phys. JETP 42, 993 (1975)].

28. V. Yu. Baranov, E. P. Velokhov, Yu. R. Kolomiiskii, V. S. Letokhov, V. G. Niz'ev, V. D. Pismenny and E. A. Ryabov, Kvantovaya Electron. (Russian) 6, 1062 (1979). 
29. V. N. Bagratashvili, V. S. Doljikov, V. S. Letokhov, A. A. Makarov, E. A. Ryabov and V. V. Tyacht, Zh. Eksp. Teor. Fiz. (Russian) 77, 2238 (1979).

30. S. Bittenson and P. L. Houston, J. Chem. Phys. 67, 4819 (1977).

31. M. Drouin, M. Gauthier, R. Pilon, P. A. Hackett and C. Wills, Chem. Phys, Lett. 60, 16 (1978).

32. P. A. Hackett, M. Gauthier, C. Wills and R. Pilon, J. Chem. Phys. 71, 546 (1979).

33. Jim-Son J. Chou and E. R. Grant, J. Chem. Phys. 74, 384 (1981).

34. J. L. Lyman and S. D. Rockwood, J. Appl. Phys. 47, 595 (1976).

35. J. L. Lyman, S. D. Rockwood and J. M. Freund, J. Chem. Phys. 67, 4545 (1977).

36. J. B. Marling and I. P. Herman, Appl. Phys. Lett. 34, 439 (1979).

37. V. V. Vizhin, Yu. N. Molin, A. K. Petrov and A. R. Sorokin, Appl. Phys. 17, 385 (1978).

38. R. V. Ambartzumian, Yu. A. Gorokhov, G. N. Makarov, A. A. Puretzky and N. P. Furzikov, Kvantovaya Electron. (Russian) 4, 1590 (1977).

39. R. V. Ambartzumian, N. P. Furzikov, Yu. A. Gorokhov, V. S. Letokhov, G. N. Makarov and A. A. Puretzky, Optics Lett. 1, 22 (1977).

40. R. V. Ambartzumian, Yu. A. Gorokhov, V. S. Letokhov and G. N. Makarov, ZhETF Pis'ma Red. 22, 96 (1975) [Sov. Phys. JETP Lett. 22, 43 (1975)].

41. V. N. Bagratashvili, Yu. R. Kolomiiskii, E. A. Ryabov and A. I. Starodubtsev, Kvantovaya Electron. (Russian) 7, 1100 (1980).

42. R. V. Ambartzumian, A. P. Dyadkin, N. P. Furzikov, A. Z. Grasyuk and B. I. Vasilyev, Appl. Phys. 15, 27 (1978).

43. J. J. Tiee and C. Wittig, J. Chem. Phys. 69, 4756 (1978).

44. R. V. Ambartzumian, N. P. Furzikov, Yu. A. Gorokhov, V. S. Letokhov, G. N. Makarov and A. A. Puretzky, Optics Commun. 18, 517 (1976).

45. I. N. Knyazev, Yu. A. Kudryavtsev, N. P. Kuz'mina and V. S. Letokhov, Zh. Eksp. Teor. Fiz. (Russian) 76, 1281 (1979).

46. R. V. Ambartzumian and V. S. Letokhov, IEEE J. Quant. Electr. QE-7, 305 (1971).

47. R. V. Ambartzumian and V. S. Letokhov, Appl. Optics 11, 354 (1972).

48. D. K. Evants, R. D. McAlpine and F. K. McClusky, Chem. Phys. 32, 81 (1978).

49. J. L. Lyman, R. J. Jensen, J. Rink and C. P. Robinson, Appl. Phys. Lett. 27, 87 (1975).

50. M. Neve de Mevergnies, Appl. Phys. Lett. 34, 853 (1979).

51. P. A. Hackett, M. Gauthier, C. Willis and R. Pilon, J. Chem. Phys. 71, 546 (1979).

52. M. Gauthier, W. S. Nip, P. A. Hackett and C. Willis, Chem. Phys. Lett. 69, 372 (1980).

53. V. S. Doljikov, Yu. R. Kolomisky and E. A. Ryabov, Chem. Phys. Lett. 80, 433 (1981).

54. R. V. Ambartzumian, V. S. Doljikov, V. S. Letokhov, E. A. Ryabov and N. V. Chekalin, Zh. Eksp. Teor. Fiz. 69, 72 (1975) [Sov. Phys. JETP 42, 36 (1975)].

55. V. M. Akulin, S. S. Alimpiev, N. V. Karlov, B. G. Sartakov and L. A. Shelepin, Zh. Eksp. Teor. Fiz. (Russian) 71, 454 (1976).

56. R. E. Huie, J. T. Herron, W. Braun and W. Tsang, Chem. Phys. Lett. 56, 193 (1978).

57. K. M. Leary, J. L. Lyman, J. B. Asprey and S. M. Freund, J. Chem. Phys. 68, 1671 (1978).

58. S. I. Linn, S. M. Lee and A. M. Ronn, Chem. Phys. Lett. 53, 260 (1978).

59. M. Gauthier, P. A. Hackett, M. Doruin, R. Pilon and C. Wills, Canad. J. Chem. 56, 2227 (1978).

60. V. V. Vizhin, V. N. Lisitsyn, A. K. Petrov and A. R. Sorokin, Izvestiya ANSSSR, ser. Khim. (Russian) N2, 485 (1977).

61. V. V. Vizhin, Yu. N. Molin, A. K. Petrov, V. N. Lisitsyn and A. R. Sorokin, React. Kin. Cat. Lett. 7, 303 (1977).

62. R. J. Jensen, J. K. Hayes, C. L. Cluff and J. M. Thorne, IEEE J. Quant. Electr. QE-16, 1352 (1980).

63. R. D. McAlpine, D. K. Evans and F. K. McClusky, Chem. Phys. 39, 263 (1979).

64. S. A. Tuccio and A. Hartford, Jr., Chem. Phys. Lett. 65, 234 (1979). 
65. Y. Makide, S. Hagiwara, O. Kurihara, K. Takeuchi, Y. Inshikawa, S. Arai, T. Tominaga, I. Inoue and R. Nakane, J. Nucl. Sci. Technol. 17, 645 (1980).

66. S. M. Freund and J. J. Ritter, Chem. Phys. Lett. 32, 255 (1975).

67. M. C. Gower and K. W. Billman, Appl. Phys. Lett. 30, 514 (1977).

68. R. V. Ambartzumian, B. I. Vasilyev, A. Z. Grasiuk, A. P. Dyadkin, V. S. Letokhov and N. P. Furzikov, Kvantovaya Electron. (Russian) 5, 1971 (1978).

69. R. V. Ambartzumian, Yu, A. Gorokhov, V. S. Letokhov, G. N. Makarov and A. A. Puretzky, Phys. Lett. 56A, 183 (1976).

70. W. Fuss and T. P. Cotter, Appl. Phys. 12, 265 (1977).

71. G. Hancock, J. D. Campbell and K. H. Welge, Optics Commun. 16, 177 (1976).

72. J. L. Lyman and K. M. Leary, J. Chem. Phys. 69, 1858 (1978).

73. J. L. Lyman, W. C. Danel, A. C. Nilsson and A. V. Novak, J. Chem. Phys. 71, 1206 (1979).

74. J. J. Tiee and C. Wittig, J. Chem. Phys. 69, 4756 (1978).

75. P. Rabinovitz, A. Kaldor, A. Gnauck, R. L. Woodin and J. S. Gethner, Optics. Lett. 7, 212 (1982). 